\title{
Sum-Frequency Generation from Chiral Media and Interfaces
}

\author{
by \\ $\mathrm{Na} \mathrm{Ji}$ \\ B.S. (University of Science and Technology of China) 2000 \\ A dissertation submitted in partial satisfaction of the \\ requirements for the degree of \\ Doctor of Philosophy \\ in \\ Chemistry \\ in the \\ GRADUATE DIVISION \\ of the \\ UNIVERSITY OF CALIFORNIA, BERKELEY
}

Committee in charge:

Professor Yuen-Ron Shen, Co-chair

Professor Richard J. Saykally, Co-chair

Professor Robert A. Harris

Professor Ivo Souza

Fall 2005 
Sum-Frequency Generation from Chiral Media and Interfaces

Copyright 2005

by

$\mathrm{Na} \mathrm{Ji}$ 


\begin{abstract}
Sum-Frequency Generation from Chiral Media and Interfaces

by

$\mathrm{Na} \mathrm{Ji}$

Doctor of Philosophy in Chemistry

University of California, Berkeley

Professor Yuen-Ron Shen, Co-chair

Professor Richard J. Saykally, Co-chair
\end{abstract}

Sum frequency generation (SFG), a second-order nonlinear optical process, is electric-dipole forbidden in systems with inversion symmetry. As a result, it has been used to study chiral media and interfaces, systems intrinsically lacking inversion symmetry. This thesis describes recent progresses in the applications of and new insights into SFG from chiral media and interfaces.

SFG from solutions of chiral amino acids is investigated, and a theoretical model explaining the origin and the strength of the chiral signal in electronic-resonance SFG spectroscopy is discussed. An interference scheme that allows us to distinguish enantiomers by measuring both the magnitude and the phase of the chiral SFG response is described, as well as a chiral SFG microscope producing chirality-sensitive images with sub-micron 
resolution. Exploiting atomic and molecular parity nonconservation, the SFG process is also used to solve the Ozma problems.

Sum frequency vibrational spectroscopy is used to obtain the adsorption behavior of leucine molecules at air-water interfaces. With poly(tetrafluoroethylene) as a model system, we extend the application of this surface-sensitive vibrational spectroscopy to fluorinecontaining polymers.

Professor Yuen-Ron Shen

Dissertation Committee Co-chair

Professor Richard J. Saykally

Dissertation Committee Co-chair 
To My Parents, Sister and Liang-shi 


\section{Contents}

List of Figures $\quad$ v

List of Tables $\quad$ ix

1 Introduction $\quad 1$

1.1 Sum-frequency generation as a probe of chirality . . . . . . . . . . . 2

1.2 Sum-frequency generation as a probe of interfaces . . . . . . . . . . 5

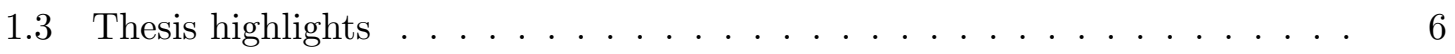

2 Sum-frequency generation spectroscopy of surfaces and chiral materials 9

2.1 Sum-frequency generation . . . . . . . . . . . . . . . 9

2.2 Sum-frequency generation spectroscopy . . . . . . . . . . . . . . 12

2.3 Sum-frequency generation in chiral liquids . . . . . . . . . . . . . 13

2.3.1 Electronic resonance and vibrational resonance cases . . . . . . . . . 15

2.3.2 Comparison of SFG with conventional techniques . . . . . . . . . 17

2.4 Sum-frequency vibrational spectroscopy (SFVS) of achiral interfaces . . . . 18

2.4 .1 Polarization combinations in SFVS . . . . . . . . . . 20

2.5 Experimental setup . . . . . . . . . . . . . . . . . . 22

3 A dynamic coupling model for sum-frequency chiral response from liquids composed of molecules with a chiral side chain and an achiral chromophore $\quad 24$

3.1 Introduction . . . . . . . . . . . . . . . . . . . . 24

3.2 Theoretical basis of SFG from isotropic chiral liquids . . . . . . . . . . . . 27

3.3 Electron correlation model . . . . . . . . . . . . . . . . . 29

3.4 Dynamic coupling model with amino acids as a model system . . . . . . . . . 32

3.5 Experimental results from amino acids with saturated side chain . . . . . . 38

3.6 Conformation analysis of amino acids . . . . . . . . . . . . . 39

3.7 Application of the dynamic coupling model to alanine, valine, leucine, and

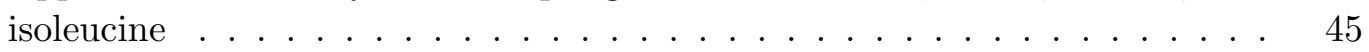

3.8 Perturbers most effective in inducing chirality . . . . . . . . . . . . 48 
3.9 Discussion on serine, threonine, and lysine . . . . . . . . . . . . 51

3.10 Experimental results on other amino acids . . . . . . . . . . . . . . 52

3.11 Additional considerations . . . . . . . . . . . . . . . . . 53

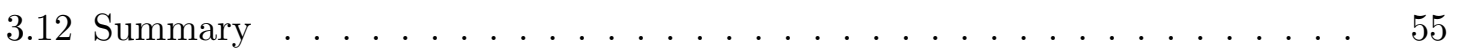

4 Towards chiral sum-frequency spectroscopy with information on both magnitude and phase $\quad 56$

4.1 Introduction . . . . . . . . . . . . . . . . . 56

4.2 Problem of optically active sum-frequency generation . . . . . . . . 57

4.3 Theory of chiral sum-frequency spectroscopy . . . . . . . . . . . . 59

4.4 Experimental arrangement . . . . . . . . . . . . . . . 61

4.5 Results and discussion . . . . . . . . . . . . . . . . . 62

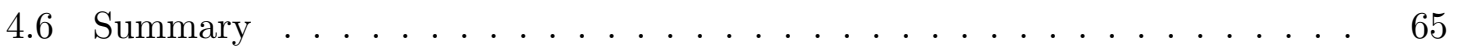

5 Three-dimensional chiral imaging by sum-frequency generation $\quad 66$

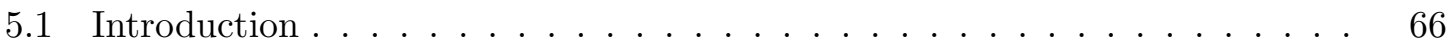

5.2 Materials and sample preparation . . . . . . . . . . . . 68

5.3 Microscope setup . . . . . . . . . . . . . . . . . . . . 69

5.4 Results and discussion . . . . . . . . . . . . . . . . . 70

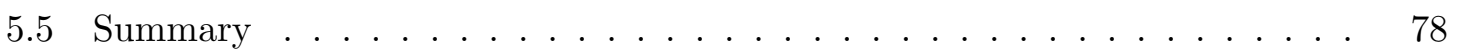

6 Atomic and molecular parity nonconservation and sum-frequency gener$\begin{array}{lr}\text { ation solutions to the Ozma problem } & \mathbf{7 9}\end{array}$

6.1 Introduction . . . . . . . . . . . . . . . . . . . . . . 79

6.2 A brief introduction to sum-frequency generation $(\mathrm{SFG}) \ldots \ldots$. . . . . 82

$6.3 \mathrm{PNC}$ and $\mathrm{SFG} \ldots \ldots \ldots \ldots \ldots \ldots \ldots \ldots$

6.4 Solutions to the second Ozma problem . . . . . . . . . . . . . . 84

6.5 Solutions to the first Ozma problem . . . . . . . . . . . . 86

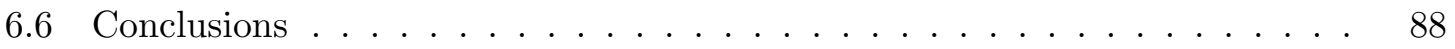

7 Sum-frequency vibrational spectroscopy of leucine molecules adsorbed at $\begin{array}{ll}\text { air-water interface } & \mathbf{9 0}\end{array}$

7.1 Introduction . . . . . . . . . . . . . . . . . . . . . . 90

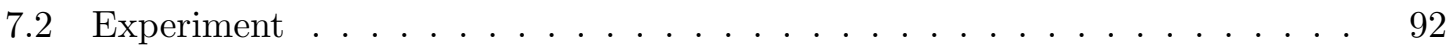

7.3 Results and discussion . . . . . . . . . . . . . . . . . . . . . . 93

7.3.1 Leucine adsorption from acidic solutions . . . . . . . . . . . 93

7.3.2 Leucine adsorption from near-neutral and basic solutions, and the comparison with acidic case . . . . . . . . . . . . 100

7.3.3 Interfacial water structure probed with SFVS . . . . . . . . . . . 105

7.4 Conclusion . . . . . . . . . . . . . . . . 107 
8 Surface vibrational spectroscopy on shear-aligned poly(tetrafluoroethylene) films

8.1 Introduction . . . . . . . . . . . . . . . . . . . . . . . . . . . . . . . . . . . . . .

8.2 Experimental results . . . . . . . . . . . . . . . . . . . . . 110

8.3 Discussion . . . . . . . . . . . . . . . . . . . . . . . 112

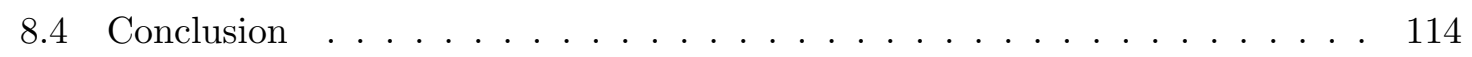

9 Conclusion $\quad 115$

$\begin{array}{ll}\text { Bibliography } & 118\end{array}$

A Sum-frequency generation described by the plane wave approximation $\begin{array}{lr}\text { and the focused Gaussian wave approximation } & \mathbf{1 2 9}\end{array}$

A.1 Collinear geometry . . . . . . . . . . . . . . . . . . . . 130

A.1.1 Plane wave description . . . . . . . . . . . . . . . . 130

A.1.2 Focused Gaussian beam description . . . . . . . . . . . . . . 133

A.2 Noncollinear geometry . . . . . . . . . . . . . . . . . . 136

B Using polarization interference to solve the first Ozma problem $\quad 138$ 


\section{List of Figures}

2.1 (a) Schematic view of the beam geometry at sample position and (b) energy level diagrams for typical electronic resonance optically active sum-frequency generation (OA-SFG) experiments. The beams at $\omega_{s}, \omega_{1}$ and $\omega_{2}$ have the same incidence plane defined by $\mathrm{X}$ and $\mathrm{Z}$ laboratory coordinate axes. Beam polarization is defined as $\mathrm{P}$ if the electric field vector $\vec{E}_{i}$ is parallel to the incidence plane, and $\mathrm{S}$ if $\vec{E}_{i}$ is perpendicular to the incidence plane. Here, electric fields are depicted in SPP polarization combination. . . . . . . . . .

2.2 (a) Schematic view of the beam geometry at sample position and (b) energy level diagrams for typical sum-frequency vibrational spectroscopy (SFVS) experiments. Beam polarization is defined as $\mathrm{P}$ if the electric field vector $\vec{E}_{i}$ is parallel to the incidence plane, and $\mathrm{S}$ if $\vec{E}_{i}$ is perpendicular to the incidence plane. . . . . . . . . . . . . . . . . .

3.1 (a) Molecular orbitals of $-\mathrm{COO}^{-}$: dots are electrons arranged in the electron configuration of the ground electronic state $|g\rangle$. (b) Energy level diagram of ${ }^{-} \mathrm{COO}^{-}$group: ground state $|g\rangle$ and the first three excited states $\left|e_{1}\right\rangle,\left|e_{2}\right\rangle$, and $\left|e_{3}\right\rangle$. The arrows denote transitions between states, to the left of which are the allowed electric-dipole and magnetic-dipole transition moments. . .

3.2 OA-SFG spectra, versus the sum-frequency wavelength, of L-enantiomers of alanine, valine, leucine, isoleucine, lysine, threonine and serine, with intensities normalized by the concentration. The OA-SFG signal from the achiral glycine is below our detection limit as expected. Lines are guides to the eye. The polarization combination used is SPP. . . . . . . . . . . .

3.3 Conformation adopted by the hydrophilic $-\mathrm{NH}_{2}$ group and $-\mathrm{COO}^{-}$group on amino acids. Here, L-alanine is presented as an example. Arrows define the molecular coordinate system $x, y, z \ldots \ldots \ldots \ldots$

3.4 Conformational isomers of alanine, valine, leucine and isoleucine. The bonds highlighted with parallel lines are the most effective perturbers in each conformer. . . . . . . . . . . . . . . . . . 
3.5 OA-SFG spectra of L-tryptophan and L-tyrosine normalized to concentration. Polarization combination is SPP. . . . . . . . . . . . .

$4.1\left|\chi_{\text {chiral }}^{(2)}\right|^{2}$ versus sum frequency wavelength from solutions of S- and R-BN. Black and gray curves are fits to the data points using Eqs. (4.2) and (4.3) with $A_{n}$ 's of the two transitions having the same and opposite signs, respectively. The polarization combination used was SPP. The molecular structure of $\mathrm{BN}$ is depicted in the inset. . . . . . . . . . . . . . .

4.2 Beam geometry of the experimental setup. Input beams were overlapped at chiral solution-quartz interface, and sum-frequency signal was detected in the transmission direction. Polarization combination was SPP. . . . . . . . .

4.3 Spectra of real and imaginary components of $\chi_{\text {chiral }}^{(2)}$ over the first two excitonsplit transitions of $(\square)$ S- and $(\mathrm{O})$ R-BN. The curves are fits to the data with Eq. (4.3) with the same set of parameters that yield the gray curve in Fig. 4.1. 63

5.1 Schematic of the microscope. . . . . . . . . . . . . . .

5.2 Spectra of (a) output sum frequency signal $\left(\omega_{s}\right)$, (b) input wave $\omega_{1}$, and (c) input wave $\omega_{2}$ from R-BN solutions. The polarization combination is SPP. The inset in (a) is the structure of a R-BN molecule. . . . . . . . . . . 5.3 OA-SFG signal dependence on (a) $I_{1}$ with $I_{2}$ constant and (b) $I_{2}$ with $I_{1}$ constant. The polarization combination is SPP. . . . . . . . . . . .

5.4 (a) Fluorescence image of a racemic BN solution and a R-BN solution separated by a $55-\mu \mathrm{m}$ glass spacer. (b) OA-SFG image of the same sample measured with SPP polarization combination. Bottom panels are signal profiles along the lines in the images. The $100 \mu \mathrm{m} \times 100 \mu \mathrm{m}$ images were taken with collection times of (a) $4 \mathrm{~ms} /$ pixel and (b) $78 \mathrm{~ms} /$ pixel and power levels of $0.24 \mathrm{~mW}\left(\omega_{1}\right)$ and $18 \mathrm{~mW}\left(\omega_{2}\right) \ldots \ldots \ldots \ldots$

5.5 SFG images of (a) one and (b) three $2.4-\mu \mathrm{m}$ diameter silica beads in R$\mathrm{BN}$ solutions. Bottom panels show signal variations along the lines in the images. The polarization combination used was SPP and the collection time per image was $78 \mathrm{~ms} /$ pixel at power levels $0.18 \mathrm{~mW}\left(\omega_{1}\right)$ and $14 \mathrm{~mW}\left(\omega_{2}\right)$.

SFG images of three $2.4-\mu \mathrm{m}$ diameter silica beads in R-BN solutions with the sample at different vertical positions. Image (b) was obtained with the SFG signal from solution maximized; Images (a) and (c) were obtained with the sample vertically displaced by $+2 \mu \mathrm{m}$ and $-2 \mu \mathrm{m}$ relative to (b), respectively. At bottom are cartoons showing the relative positions of the interaction region (black) and the sample (grey for fused silica slides and beads, white for R-BN solutions). Black arrows denote scanning direction. The polarization combination is SPP and the collection time is $78 \mathrm{~ms} /$ pixel at power $0.24 \mathrm{~mW}$ $\left(\omega_{1}\right)$ and $18 \mathrm{~mW}\left(\omega_{2}\right) \ldots \ldots \ldots \ldots \ldots$

6.1 Wizard of OZ needs to decide which side of tin woodman's chest to put the heart in. Image adapted from the original drawing by W. W. Denslow in "The Wizard of Oz". . . . . . . . . . . . . . . . 
6.2 Experimental configurations 1 and 2. The electric field $\vec{E}$ is normal to the incident plane defined by the wave vectors $\vec{k}_{1}, \vec{k}_{2}$, and $\vec{k}_{3}$. Polarizations for the three waves are $\vec{S}_{1}, \vec{P}_{2}$ and $\vec{P}_{3}$, respectively, as defined in each configuration. Image adapted from the original drawing by W. W. Denslow in "The Wizard of $\mathrm{Oz} " \ldots \ldots \ldots \ldots \ldots \ldots \ldots$

7.1 SFVS spectra of leucine molecules adsorbed at air-water interface from acidic ( $\mathrm{pH} \sim 0.1)$ solutions with different concentrations. The polarization combinations used were $\mathrm{S}(\mathrm{SF}) \mathrm{S}$ (visible)P(IR), SPS and PPP. Lines are the fitted curves using Eqs. (2.3), (2.4) and (2.18) . . . . . . . . . . . . .

7.2 Geometry that defines the orientational angles $\phi, \theta$ and $\psi$ of the head groups of leucine molecules $\left(\mathrm{CH}_{3}\right)_{2} \mathrm{CHCH}_{2} \mathrm{CH}\left(\mathrm{NH}_{2}\right) \mathrm{COOH}$ adsorbed at air-water interfaces. . . . . . . . . . . . . . . . .

7.3 (a) Surface density $N_{\mathrm{S}}$ 's versus bulk concentration for acidic solutions. The line is a fitted Langmuir isotherm. (b) Dependence of orientational angles $\theta$ and $\psi$ on surface density for the acidic solution. The surface densities are (A) $3.6 \pm 0.1$, (B) $3.6 \pm 0.1$, (C) $3.2 \pm 0.1$, (D) $2.8 \pm 0.1$, and (E) $2.5 \pm 0.3$ in units of $10^{14} \mathrm{~cm}^{-2} \ldots \ldots \ldots \ldots \ldots$

7.4 SFVS spectra of leucine molecules adsorbed at air-water interface from (a) near-neutral $(\mathrm{pH} \sim 5.9)$ and $(\mathrm{b})$ basic $(\mathrm{pH} \sim 13.1)$ solutions with different concentrations. The polarization combinations used were SSP, SPS and PPP. Lines are fitted curves using Eqs. (2.3), (2.4) and (2.18) . . . . . . . . .

7.5 SFVS spectra of leucine molecules adsorbed from nearly saturated (a) acidic, (b) near-neutral and (c) basic solutions, together with the simulated SFVS spectra that are free of the nonresonant background for (d) acidic, (e) nearneutral and (f) basic solutions. Polarization combination used was SSP. . .

7.6 SFVS spectra of water at air-water interfaces with and without adsorbed leucine molecules. The bulk $\mathrm{pH}$ values of the solutions are (a) $\sim 0.1$, (b) $\sim 5.9$, and $(\mathrm{c}) \sim 13.1$. Schematics on the side depict the interfacial water structure upon the adsorption of leucine molecules. The circles stand for the hydrophilic part of leucine molecules, and the rectangles depict the hydrophobic part. Polarization combination used was SSP. The solid lines are guides to the eye. . . . . . . . . . . . . . . . .

8.1 SFVS spectra from a shear-deposited thin film of PTFE. The polarization combination used was $\mathrm{S}(\mathrm{SFG}) \mathrm{P}($ Visible)S(Infrared). . . . . . . . . . . . .

8.2 Schematic showing a PTFE chain aligned along the shearing direction in the incident plant. Electric field directions of the beams as marked are of SPS polarization combination. . . . . . . . . . . . . . 
A.1 Curves showing the variation of sum-frequency electric field with interaction length $l$ for planar input waves. From left to right, the interaction length $l$ 's are such that $\Delta k l$ equals to $\pi / 2, \pi, 3 \pi / 2$, and $2 \pi$, respectively. The lengths of the gray arrows reflect the amplitudes of the generated sum-frequency electric field after interaction length $l \ldots \ldots \ldots \ldots$

A.2 (a) Incident waves with their focal planes at $Z=0$. (b) Spirals showing the generation of sum-frequency field in the nonlinear medium from $\mathrm{Z}=-\infty$ to $\mathrm{Z}=0$ (upper spiral) and $\mathrm{Z}=0$ to $\mathrm{Z}=+\infty$ (lower spiral). The thick black arrows indicate the relative phase and amplitude of the electric fields in two

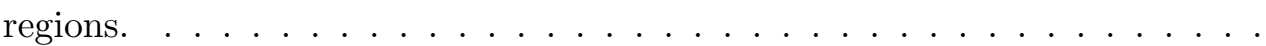

A.3 (a) Interaction volume in nonlinear geometry with three planes A, B, and C defined as its starting, bisecting, and ending planes. (b) Spirals describe the sum-frequency electric fields generated in regions from plane A to plane B (upper spiral) and from plane B to plane C (lower spiral). . . . . . . . . . 137

B.1 Two experimental configurations (1 and 2) for wave vectors $\vec{k}_{1}, \vec{k}_{2}$, and $\vec{k}_{3}$, with the latter two waves having $\vec{P}$ polarizations $\left(\vec{P}_{2}\right.$ and $\left.\vec{P}_{3}\right)$. The polarization for $\vec{k}_{1}$ (thick dotted lines) is set to be $45^{\circ}$ away from $\vec{S}_{1}$, with its upper part closer to the tin woodman than its bottom part. . . . . . . . . . . 


\section{List of Tables}

3.1 Polarizabilities of C-C and C-H bonds from literature (in units of $\AA^{3}$ ) . . 45

3.2 Calculated perturbation strength $\left(\Omega\right.$ in units of $\AA^{-1}$ ) for 15 conformers of L-alanine, L-valine, L-leucine, and L-isoleucine using different bond polarizability values ${ }^{a} \ldots \ldots \ldots \ldots \ldots \ldots$. . . . . . . . . . . . . 46

7.1 Frequencies, damping constants, and assignment of modes . . . . . . . . .

7.2 Real and imaginary part $\left(10^{-22} \mathrm{~m}^{2} \mathrm{~V}^{-1}\right)$ of nonresonant background $\chi_{\mathrm{NR}}^{(2)}$ at $2960 \mathrm{~cm}^{-1}$ obtained from fitting for SSP spectra of three near-saturated solutions with different $\mathrm{pH}$ values (Both the real and imaginary part are assumed to change linearly with infrared wavenumber in the range 2800-3000 $\mathrm{cm}^{-1}$. . . . . . . . . . . . . . . . . . .

7.3 Deduced orientational angles $\theta$ and $\psi$ for leucine molecules adsorbed at airwater interfaces from near-neutral and basic solutions . . . . . . . . . . 102 


\section{Acknowledgments}

I consider myself very lucky to have come to Berkeley for graduate school. Here

I spent five wonderful years working with a very talented group of people. All the work described in this thesis would not have been possible without them.

It is difficult to find words to sufficiently express my gratitude to my advisor, Prof. Yuen-Ron Shen. He is my role model: his extraordinary curiosity and creativity in science, his clarity in thought and communication, his kindness and patience as an advisor, and his generosity with his time and advice whenever needed set an extremely high standard, which I will strive to meet in my future career. The support, training, and trust he gave me throughout my years as a graduate student will be forever appreciated and cherished.

I could not ask for better colleagues than the students and postdoctoral fellows in Shen group. Besides being brilliant scientists, they are also very fine human beings, which makes the long hours in the basement a true enjoyment. Mikhail Belkin, a fellow graduate student, was my mentor when I started working on the chirality projects. Besides all the instrumentation knowledge he taught me, his "Russian" approach to experiments and his inquisitive spirit strongly influenced me as a scientist. Dr. Victor Ostroverkhov, a wonderful colleague and a close friend, has been a rich source of knowledge in optics, electronics and computer softwares. His ingenuity in setup-building is only matched by his willingness to share knowledge and to help others. Outside the lab, his appreciation in many aspects of life has greatly influenced me and helped me lead a more balanced life. Dr. François Lagugné-Labarthet was one of my collaborators on the polymer project. His 
enthusiasm for both science and life is infectious and his cheerfulness made many long days of building an OPG/OPA setup in a dark room pleasant. It is truly enjoyable to share offices with John Mcguire and Thai Truong, two graduate students who always bear with my entropy-maximized desks. John is the master of the femtosecond laser, from whom there is always something to learn. Thai's kindness and consideration towards others make sharing experimental setups with him a wonderful experience. My thanks also go to other members, past and present, of Shen group: especially, Dr. Chun Zhang, who helped me start in the group, Dr. Xing Wei, Prof. Lei Xu, Dr. Markus Raschke, Dr. Songhee Han, Dr. Seok-Cheol Hong, Dr. Ying-Jen Shiu, Gene-wei Li, a very talented undergraduate student, Dr. Pasquale Pagliusi, who taught me the importance of proper dinners, Eric (Chao-Yuan) Chen, an exchange student with many talents and our tireless badminton coach, Dr. Feng Wang, Dr. Evgenia Kim, Weitao Liu, and Luning Zhang.

I benefit greatly from collaboration with colleagues outside Shen group. I thank Prof. Richard Saykally for agreeing to be my co-advisor. Collaboration with Prof. Haw Yang and his graduate student Kai Zhang on the microscopy project has been a very fruitful and pleasant experience. My interaction with Prof. Robert Harris, first as a teaching assistant then as a collaborator, is very enlightening and enjoyable. Bob became a great friend, with whom I can discuss many topics beyond science.

Finally, I want to thank my parents and my sister for teaching me that the most important thing in life is to do what one loves with a peaceful mind. My coming to Berkeley resulted in meeting Liang-shi, whose unbeatable optimism and confidence has supported me throughout my years in Berkeley. This thesis is dedicated to them. 


\section{Chapter 1}

\section{Introduction}

Sum-frequency generation (SFG) is a second-order nonlinear optical process, in which electromagnetic radiation with frequency $\omega_{s}=\omega_{1}+\omega_{2}$ is generated when two input electromagnetic waves with frequencies $\omega_{1}$ and $\omega_{2}$ interact in a medium. Quantum mechanically, SFG can be described by second-order perturbation theory with the interaction Hamiltonian being

$$
H_{\mathrm{int}}=-e \vec{r} \cdot \vec{E}
$$

in the electric dipole approximation [1]. Within this approximation, only in systems without inversion symmetry is SFG allowed. The applications of this property transformed SFG from a laboratory novelty to a powerful analytical technique capable of providing molecular level information that is otherwise inaccessible. In this thesis, we describe some recent developments in the applications as well as theoretical understanding of SFG from chiral media and interfaces, systems that naturally lack inversion symmetry. 


\subsection{SUM-FREQUENCY GENERATION AS A PROBE OF CHIRALITY 2}

\subsection{Sum-frequency generation as a probe of chirality}

The property that a 3-dimensional (3D) object cannot overlap with its mirror image was named "chirality" by Lord Kelvin [2]:

"I call any geometrical figure, or group of points, chiral, and say it has chirality if its image in a plane mirror, ideally realized, cannot be brought to coincide with itself."

In terms of symmetry, chirality is the absence of symmetry under improper rotations. In other words, a chiral object should not have a symmetry plane or inversion center. Our hands are chiral - left hand is the mirror image of right hand, but does not superpose the right hand. An enantiomer is one of a pair of chiral compounds (crystals or molecules) that are mirror images of each other. A pair of enantiomers are said to have opposite handedness.

Chirality is a ubiquitous property in our biological world. The function of the fundamental components of life - proteins, nucleic acids, and sugar - relies on their being chiral. The handedness of a molecule affects its odor, taste, and toxicity. Therefore, the ability to distinguish enantiomers is crucial in developing new compounds for food and pharmaceutical applications.

Techniques most commonly used to detect chirality are optical techniques, such as circular dichroism (CD), optical rotatory dispersion (ORD), vibrational circular dichroism (VCD), and Raman optical activity (ROA) [3]. These techniques measure the differences in the response functions of the sample towards left and right circularly polarized light, with the differences being of opposite signs for enantiomers. Because these responses are enhanced when the probing beam is in resonance with a molecular transition, besides being 


\subsection{SUM-FREQUENCY GENERATION AS A PROBE OF CHIRALITY 3}

able to distinguish enantiomers, these techniques can provide spectroscopic information, which makes them extremely useful for molecular characterization.

The differences detected by the conventional techniques are only nonzero, however, due to the higher order interactions of molecules and light. To describe them, the interaction Hamiltonian in Eq. (1.1) has to include extra coupling terms of the molecular magnetic dipole and electric quadrupole moments with the electromagnetic wave [4]. As a result, the differences are in the order of $10^{2}$ to $10^{5}$ times smaller than usual responses arising from the electric dipole term in Eq. (1.1). Consequently, the sensitivity of these techniques is limited, and it is difficult to use them to detect chirality from systems such as monolayers or thin films.

The advent of new technologies, such as combinatorial chemistry [5] or lab-ona-chip technologies [6], requires rapid screening and testing of chemicals of often small quantities, sometimes down to the monolayer level. Moreover, many biological processes involve molecules that either function only when imbedded in a membrane, such as membrane proteins, or accumulate and interact mainly at interfaces [7]. A sensitive probe that allows in situ studies of molecular chirality of such systems would open up new research opportunities and provide new understanding of molecular chirality.

In our laboratory, SFG has been developed as an alternative spectroscopic tool to probe molecular chirality. Unlike the conventional methods, SFG is allowed in the electric dipole approximation in chiral materials. Thus, it can have higher sensitivity than the conventional techniques in detecting chirality. Because SFG is also enhanced when resonance condition is satisfied, by using tunable inputs, one can obtain vibrational and electronic 


\subsection{SUM-FREQUENCY GENERATION AS A PROBE OF CHIRALITY 4}

spectra of the chiral materials.

Second harmonic generation (SHG), a special case of SFG with the two input waves of the same frequency, is forbidden in chiral liquids. However, it can probe the chirality of anisotropic systems such as chiral surfaces, as first demonstrated by Hicks group $[8,9,10,11$, 12]. Reviews on SHG as a probe for surface chirality can be found in references $[13,14,15]$.

Although SFG in chiral liquids was theoretically investigated as early as 1965 [16], it was only in 2000 that our group demonstrated this effect conclusively [17]. Since then, we have obtained chiroptical electronic and vibrational spectra from chiral liquids $[18,19,20$, 21], thin films [22], and monolayers of chiral molecules [19, 23]. Using a double-resonance scheme, we have obtained an optically active vibrational spectrum from a molecular monolayer for the first time [19].

It is our goal to develop SFG as a novel spectroscopic method for probing molecular chirality that can satisfy the sensitivity requirement of modern science and technology, and to provide basic understanding of its underlying principles. In this thesis, our recent efforts toward this goal are described. In particular, we expanded the application of SFG to the study of chiral biological molecules, starting with amino acids; we also developed a technique that allows us to obtain both the phase and the amplitude of the chiral SFG response coefficients; we implemented chirality-sensitive SFG in a microscopic configuration with the goal of imaging cells with chiral contrast. We investigated theoretically the mechanism of SFG from chiral liquids made of molecules with an achiral chromophore and a chiral side chain. Together with parity nonconservation, SFG was used to solve the Ozma problem. 


\subsection{SUM-FREQUENCY GENERATION AS A PROBE OF INTERFACES 5}

\subsection{Sum-frequency generation as a probe of interfaces}

SFG can be used as a probe of interfaces between materials that have inversion symmetry in bulk. Because inversion symmetry is always broken at the interfaces, for these systems SFG is surface-sensitive within electric-dipole approximation. In 1987, Our group first demonstrated SFG as a surface sensitive spectroscopic technique with sub-monolayer sensitivity $[24,25,26]$. Compared with traditional techniques in surface science, such as those utilizing the limited penetration depths of probe particles (X-ray photons, electrons, atoms, ions, or neutrons) to probe structures close to the surfaces, SFG does not need ultrahigh vacuum, is more surface specific, and through resonant enhancement, can provide chemical information. As an optical method, SFG can be used to study any interfaces that are accessible to light, including buried interfaces and liquid surfaces, which are difficult to study using other techniques. As a result, since 1987, there have been around two thousand papers using SFG to study various interfaces important for fundamental understanding as well as technical applications.

This thesis gives two examples of using sum-frequency generation to obtain the vibrational spectra of surface species. One study is on the adsorption behavior of leucine molecules as well as the structure of surface water molecules perturbed by leucine adsorption. In the other study, we expanded the application of SFG to C-F vibrational modes and studied the surface structure of shear-aligned poly(tetrafluoroethylene) films. 


\subsection{Thesis highlights}

This thesis is organized as follows.

In Chapter 2, we describe the basic theory of SFG and show how it can be used to measure chirality of liquids and to obtain surface vibrational spectra.

In Chapter 3, we present optically active sum-frequency generation (OA-SFG) spectra of a series of amino acids near the electronic resonance of their intrinsically achiral carboxyl group. Adapting an electron correlation model of linear optical activity, we present a dynamic coupling model for OA-SFG near the electronic resonance of the achiral chromophore. Our model shows that the nonlinear chiroptical response comes about by the through-space correlative electronic interactions between the chiral side chain and the achiral chromophore, and its magnitude is determined by the position and orientation of the bonds that make up the chiral side chain. Using the bond polarizability values in the literature and the conformations of amino acids obtained from calculation, we can reproduce the relative OA-SFG strength of a series of amino acids.

In Chapter 4, a novel chiral sum-frequency spectroscopy with both magnitude and phase information is described. Chiral spectra of both real and imaginary components of the nonlinear chiroptical response over the first exciton-split transitions of enantiomers of 1,1'bi-2-naphthol were obtained through interferences with a dispersionless background. The availability of phase information should allow us to determine the absolute configuration and conformation of chiral molecules.

In Chapter 5, we describe the first sum-frequency generation microscope that is 
sensitive toward molecular chirality. Optically active images of chiral 1,1'-bi-2-naphthol solutions were obtained with sub-micron spatial resolution. Three dimensional sectioning capability of our microscope was also demonstrated. This optically active SFG microscopy can potentially become a powerful imaging technique for biological samples.

In Chapter 6, we define two Ozma problems, which have to do with how to communicate a chiral reference system through an achiral channel. Parity nonconservation is necessary for their solutions. Although both problems may be solved by the formalisms of $\beta$-decay or atomic optical activity, atomic and molecular sum-frequency generation is chosen, as it supplies rich methods of effecting gedanken solutions to the Ozma problems. A new method of measuring a parameter manifesting molecular parity violations is advanced.

In Chapter 7, sum-frequency vibrational spectroscopy is used to study adsorption of leucine molecules at air-water interface from solutions with different concentrations and $\mathrm{pH}$ values. The surface density and the orientation of the isopropyl head group of the adsorbed leucine molecules could be deduced from the measurements. We find that the orientation depends on the surface density, but only weakly on bulk $\mathrm{pH}$ at the saturated surface density. The vibrational spectra of the interfacial water molecules are strongly affected by the charge state of the adsorbed leucine molecules. Enhancement and inversion of polar orientation of interfacial water molecules by surface charges or field controllable by the bulk $\mathrm{pH}$ value are observed.

In Chapter 8, sum-frequency vibrational spectroscopy is used to obtain the first surface vibrational spectra of shear-deposited highly-oriented poly(tetrafluorothylene) thin films. The surface poly(tetrafluorothylene) chains appear to lie along the shearing direction. 
Vibrational modes observed at 1142 and $1204 \mathrm{~cm}^{-1}$ are found to have the $\mathrm{E}_{1}$ symmetry, in support of some earlier analysis in the long-lasting controversy over the assignment of these modes. 


\section{Chapter 2}

\section{Sum-frequency generation}

\section{spectroscopy of surfaces and chiral}

\section{materials}

\subsection{Sum-frequency generation}

When two electromagnetic waves with frequencies $\omega_{1}$ and $\omega_{2}$ interact in a macro-

scopic medium, a nonlinear polarization $\vec{P}^{(2)}\left(\omega_{s}=\omega_{1}+\omega_{2}\right)$ can be induced. Coherent radiation at frequency $\omega_{s}$ resulting from this nonlinear polarization obeys the wave equation

$$
\nabla \times \nabla \times \vec{E}_{s}+\frac{\epsilon}{c^{2}} \frac{\partial^{2} \vec{E}_{s}}{\partial t^{2}}=-\frac{4 \pi}{c^{2}} \frac{\partial^{2} \vec{P}^{(2)}\left(\omega_{s}\right)}{\partial t^{2}}
$$


If we only consider the electric-dipole interaction between the light and the medium, the value of this polarization is determined by the medium's second-order nonlinear susceptibility $\overleftrightarrow{\chi}^{(2)}$ and the electric fields of the input waves $\vec{E}_{1}, \vec{E}_{2}$ with

$$
\vec{P}^{(2)}\left(\omega_{s}\right)=\overleftrightarrow{\chi}^{(2)}\left(\omega_{s}\right): \vec{E}_{1} \vec{E}_{2}
$$

If the medium has inversion symmetry, it can be shown that all components of $\overleftrightarrow{\chi}^{(2)}$ become zero. Thus under the electric-dipole approximation, SFG is only allowed in media without inversion symmetry, such as surfaces and chiral media.

With Eq. (2.2), Eq. (2.1) can be solved. Solutions have been worked out and will not be reproduced here $[1,27]$. Some computational details are presented in Appendix A, which have to do with practical considerations in experiments. Here, only equations bearing direct relationship to experimental results are shown.

If we direct two input waves from air to a medium and approximate the input waves as plane waves, the output sum-frequency wave is also a plane wave with the intensity

$$
I\left(\omega_{s}\right)=\frac{\omega_{s}^{2}}{8 \epsilon_{0} c^{3} \cos ^{2} \beta_{s}}\left|\chi_{\mathrm{eff}}^{(2)}\right|^{2} I\left(\omega_{1}\right) I\left(\omega_{2}\right)
$$

where $I\left(\omega_{1}\right)$ and $I\left(\omega_{2}\right)$ are the intensities of the input fields, $\beta_{s}$ is the exit angle of the sum-frequency wave in air relative to surface normal, and $\chi_{\text {eff }}^{(2)}$ is the effective second-order nonlinear susceptibility defined as [28]

$$
\chi_{\mathrm{eff}}^{(2)}=\left[\overleftrightarrow{L}\left(\omega_{s}\right) \cdot \hat{e}_{s}\right] \cdot \stackrel{\leftrightarrow}{\chi}(2):\left[\overleftrightarrow{L}\left(\omega_{1}\right) \cdot \hat{e}_{1}\right]\left[\overleftrightarrow{L}\left(\omega_{2}\right) \cdot \hat{e}_{2}\right]
$$

where $\overleftrightarrow{L}\left(\omega_{i}\right)$ and $\hat{e}_{i}$ are the tensorial Fresnel factor and the unit polarization vector of the optical field at $\omega_{i}$, respectively. $\overleftrightarrow{L}\left(\omega_{i}\right)$ describes the relationship between field components 
in the air and in the medium [29]. For isotropic media, only the diagonal elements of $\overleftrightarrow{L}\left(\omega_{i}\right)$ need to be considered, which are

$$
\begin{aligned}
L_{\mathrm{XX}} & =\frac{2 n_{1} \cos \alpha_{2}}{n_{1} \cos \alpha_{2}+n_{2} \cos \alpha_{1}} \\
L_{\mathrm{YY}} & =\frac{2 n_{1} \cos \alpha_{1}}{n_{1} \cos \alpha_{1}+n_{2} \cos \alpha_{2}} \\
L_{\mathrm{ZZ}} & =\frac{2 n_{2} \cos \alpha_{1}}{n_{1} \cos \alpha_{2}+n_{2} \cos \alpha_{1}}\left(\frac{n_{1}}{n^{\prime}}\right)^{2},
\end{aligned}
$$

where $n_{1}, n_{2}$ and $\alpha_{1}, \alpha_{2}$ are the refractive indices and incident angles in the air and in the medium, respectively. For the case of an interfacial medium, $n^{\prime}$ is the effective refractive index of the surface; for the case of a bulk sample, $n^{\prime}$ equals to the bulk refractive index $n_{2} . \mathrm{X}, \mathrm{Y}, \mathrm{Z}$ are the laboratory coordinate axes with $\mathrm{Z}$ defined to be normal to the surface of the medium and $\mathrm{X}-\mathrm{Z}$ plane the incidence plane.

For an interfacial system, $\overleftrightarrow{\chi}^{(2)}$ in Eq. (2.4) is the surface second-order nonlinear susceptibility tensor $\overleftrightarrow{\chi}_{\mathrm{S}}^{(2)}$; for a bulk system, $\stackrel{\leftrightarrow}{\chi}(2)$ is $\overleftrightarrow{\chi}_{\mathrm{B}}^{(2)} / \Delta k_{\mathrm{Z}}$, with $\overleftrightarrow{\chi}_{\mathrm{B}}^{(2)}$ being the bulk second-order nonlinear susceptibility tensor [1]. $\Delta k_{\mathrm{Z}}=k_{1, \mathrm{Z}}+k_{2, \mathrm{Z}}-k_{s, \mathrm{Z}}$ describes the wavevector mismatch along $\mathrm{Z}$ direction, the inverse of which is the effective coherence length $l_{c}$ with

$$
l_{c}=\frac{1}{\left|k_{1, \mathrm{Z}}+k_{2, \mathrm{Z}}-k_{s, \mathrm{Z}}\right|}=\frac{1}{\left|\Delta k_{\mathrm{Z}}\right|} .
$$

Physically, coherence length $l_{c}$ can be considered as the interaction length within which the sum-frequency waves generated at different positions interfere constructively. 


\subsection{Sum-frequency generation spectroscopy}

Microscopically, the second-order nonlinear susceptibility $\stackrel{\leftrightarrow}{\chi}^{(2)}$ is related to the second-order nonlinear polarizability $\stackrel{\leftrightarrow}{\alpha}^{(2)}$ (first hyperpolarizability) by the expression [1]

$$
\chi_{i j k}^{(2)}=\frac{1}{\epsilon_{0}} N l_{i i}\left(\omega_{s}\right) l_{j j}\left(\omega_{1}\right) l_{k k}\left(\omega_{2}\right) \sum_{i^{\prime} j^{\prime} k^{\prime}} \alpha_{i^{\prime} j^{\prime} k^{\prime}}^{(2)}\left\langle\left(\hat{i} \cdot \hat{i}^{\prime}\right)\left(\hat{j} \cdot \hat{j}^{\prime}\right)\left(\hat{k} \cdot \hat{k}^{\prime}\right)\right\rangle .
$$

Here $N$ is the number density of molecules, $\overleftrightarrow{l}\left(\omega_{i}\right)$ is the microscopic local field correction factor tensor of field $\omega_{i}[30], \hat{i}, \hat{j}, \hat{k}$ refer to the laboratory coordinates (X,Y,Z), $\hat{i}^{\prime}, \hat{j}^{\prime}, \hat{k}^{\prime}$ refer to the molecular coordinates $(x, y, z)$, and the angular brackets denote an average over the molecular orientations.

The quantum mechanical expression of $\stackrel{\leftrightarrow}{\alpha}^{(2)}$ is

$$
\begin{aligned}
& \alpha_{i j k}^{(2)}=\frac{1}{\hbar^{2}} \sum_{n, n^{\prime}} \frac{\left(\mu_{i}\right)_{g n}\left(\mu_{j}\right)_{n n^{\prime}}\left(\mu_{k}\right)_{n^{\prime} g}}{\left(\omega_{s}-\omega_{n g}+i \Gamma_{n g}\right)\left(\omega_{2}-\omega_{n^{\prime} g}+i \Gamma_{n^{\prime} g}\right)}+\frac{\left(\mu_{i}\right)_{g n}\left(\mu_{j}\right)_{n^{\prime} g}\left(\mu_{k}\right)_{n n^{\prime}}}{\left(\omega_{s}-\omega_{n g}+i \Gamma_{n g}\right)\left(\omega_{1}-\omega_{n^{\prime} g}+i \Gamma_{n^{\prime} g}\right)}+ \\
& +\frac{\left(\mu_{i}\right)_{n g}\left(\mu_{j}\right)_{n^{\prime} n}\left(\mu_{k}\right)_{g n^{\prime}}}{\left(\omega_{s}+\omega_{n g}+i \Gamma_{n g}\right)\left(\omega_{2}+\omega_{n^{\prime} g}+i \Gamma_{n^{\prime} g}\right)}+\frac{\left(\mu_{i}\right)_{n g}\left(\mu_{j}\right)_{g n^{\prime}}\left(\mu_{k}\right)_{n^{\prime} n}}{\left(\omega_{s}+\omega_{n g}+i \Gamma_{n g}\right)\left(\omega_{1}+\omega_{n^{\prime} g}+i \Gamma_{n^{\prime} g}\right)}- \\
& -\frac{\left(\mu_{i}\right)_{n^{\prime} n}\left(\mu_{j}\right)_{n g}\left(\mu_{k}\right)_{g n^{\prime}}}{\left(\omega_{s}-\omega_{n n^{\prime}}+i \Gamma_{n n^{\prime}}\right)} \cdot\left(\frac{1}{\omega_{2}+\omega_{n^{\prime} g}+i \Gamma_{n^{\prime} g}}+\frac{1}{\omega_{1}-\omega_{n g}+i \Gamma_{n g}}\right)- \\
& -\frac{\left(\mu_{i}\right)_{n^{\prime} n}\left(\mu_{j}\right)_{g n^{\prime}}\left(\mu_{k}\right)_{n g}}{\left(\omega_{s}-\omega_{n n^{\prime}}+i \Gamma_{n n^{\prime}}\right)} \cdot\left(\frac{1}{\omega_{2}-\omega_{n g}+i \Gamma_{n g}}+\frac{1}{\omega_{1}+\omega_{n^{\prime} g}+i \Gamma_{n^{\prime} g}}\right) \text {, }
\end{aligned}
$$

where $n, n^{\prime}$ denote the eigenstates of the molecule other than the ground state $|g\rangle, i, j$, and $k$ refer to the molecular coordinates, $(\vec{\mu})_{a b}, \omega_{a b}$ and $\Gamma_{a b}$ are the electric-dipole transition moment, the transition frequency, and the damping constant of the transition $|a\rangle \rightarrow|b\rangle$, respectively. Here all the molecules are assumed to be initially in the ground state $|g\rangle$.

From Eqs. (2.9) and (2.10), one sees that SFG can be used to obtain spectroscopic information, because when one of the three waves is in resonance with a molecular transition, 
components of $\overleftrightarrow{\chi}^{(2)}$ are enhanced. A general expression describing such a resonance behavior is

$$
\overleftrightarrow{\chi}^{(2)}=\overleftrightarrow{\chi}_{\mathrm{NR}}^{(2)}+\overleftrightarrow{\chi}_{\mathrm{R}}^{(2)}=\overleftrightarrow{\chi}_{\mathrm{NR}}^{(2)}+\sum_{q} \frac{\overleftrightarrow{A}_{q}}{\omega_{i}-\omega_{q}+i \Gamma_{q}}
$$

where $\overleftrightarrow{\chi}_{\text {NR }}$ is the nonresonant part of $\overleftrightarrow{\chi}^{(2)}$ while $\overleftrightarrow{A}_{q}, \omega_{q}$, and $\Gamma_{q}$ are the amplitude, the resonant frequency, and the damping constant of the transition $q$, respectively.

Experimentally, the frequency of one input wave is tuned so that this wave or the sum-frequency wave is in resonance with a vibrational or electronic transition, and the resonant enhancement of the sum-frequency output gives us the vibrational or electronic spectra of the medium.

\subsection{Sum-frequency generation in chiral liquids}

Liquids made of chiral molecules of the same handedness do not have inversion symmetry, thus SFG is allowed in chiral liquids. Due to the isotropy of liquids, the nonvanishing $\overleftrightarrow{\chi}^{(2)}$ elements have the same amplitude and are related by

$$
\chi_{\mathrm{B}, \mathrm{XYZ}}^{(2)}=-\chi_{\mathrm{B}, \mathrm{XZY}}^{(2)}=\chi_{\mathrm{B}, \mathrm{YZX}}^{(2)}=-\chi_{\mathrm{B}, \mathrm{YXZ}}^{(2)}=\chi_{\mathrm{B}, \mathrm{ZXY}}^{(2)}=-\chi_{\mathrm{B}, \mathrm{ZYX}}^{(2)}=\chi_{\mathrm{chiral}}^{(2)} .
$$

They are of opposite signs for the two enantiomers and zero for a racemic mixture.

For chiral liquids, $\chi_{\text {chiral }}^{(2)}$ is related to the first molecular hyperpolarizability by [1]

$$
\chi_{\text {chiral }}^{(2)}=\frac{1}{\epsilon_{0}} N_{\mathrm{B}} l_{\mathrm{B}}\left(\omega_{s}\right) l_{\mathrm{B}}\left(\omega_{1}\right) l_{\mathrm{B}}\left(\omega_{2}\right) \alpha_{\text {chiral }}^{(2)}
$$



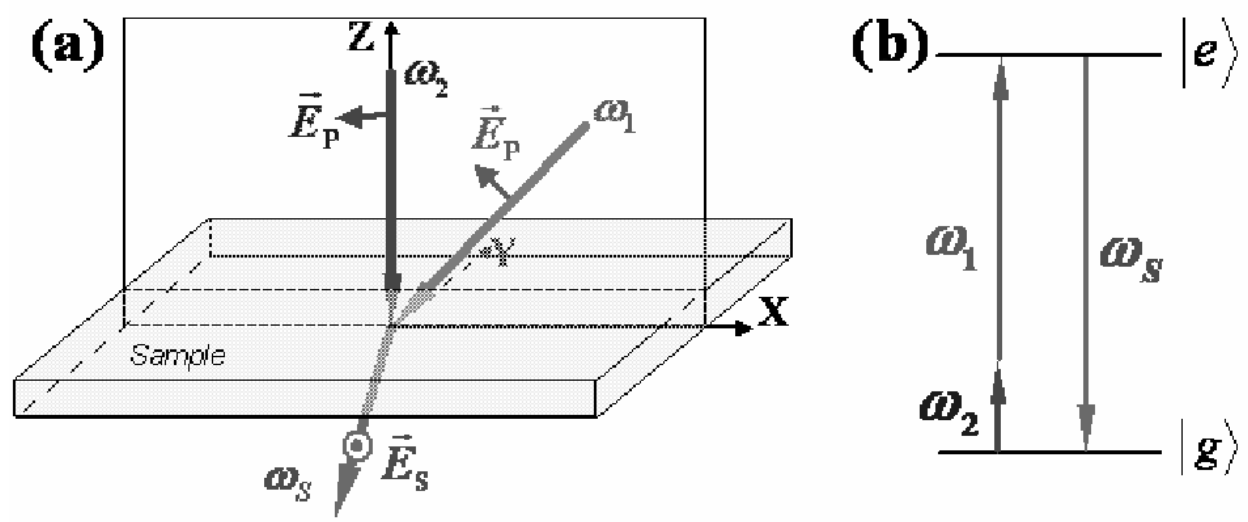

Figure 2.1: (a) Schematic view of the beam geometry at sample position and (b) energy level diagrams for typical electronic resonance optically active sum-frequency generation (OA-SFG) experiments. The beams at $\omega_{s}, \omega_{1}$ and $\omega_{2}$ have the same incidence plane defined by $\mathrm{X}$ and $\mathrm{Z}$ laboratory coordinate axes. Beam polarization is defined as $\mathrm{P}$ if the electric field vector $\vec{E}_{i}$ is parallel to the incidence plane, and $\mathrm{S}$ if $\vec{E}_{i}$ is perpendicular to the incidence plane. Here, electric fields are depicted in SPP polarization combination.

where

$$
\begin{aligned}
\alpha_{\text {chiral }}^{(2)} & \equiv \sum_{i, j, k} \alpha_{i j k}^{(2)}\langle(\hat{\mathrm{X}} \cdot \hat{i})(\hat{\mathrm{Y}} \cdot \hat{j})(\hat{\mathrm{Z}} \cdot \hat{k})\rangle_{\text {iso }} \\
& =\frac{1}{6}\left[\alpha_{x y z}^{(2)}-\alpha_{y x z}^{(2)}+\alpha_{y z x}^{(2)}-\alpha_{z y x}^{(2)}+\alpha_{z x y}^{(2)}-\alpha_{x z y}^{(2)}\right]
\end{aligned}
$$

Here $N_{\mathrm{B}}$ is the number density of chiral molecules, $\mathrm{X}, \mathrm{Y}$, and $\mathrm{Z}$ are the laboratory coordinates, $l_{\mathrm{B}}\left(\omega_{i}\right)$ is the bulk microscopic local field factor with $l_{\mathrm{B}}\left(\omega_{i}\right)=\left[\epsilon\left(\omega_{i}\right)+2\right] / 3\left(\epsilon\left(\omega_{i}\right)\right.$ being the dielectric constant of the liquid at $\left.\omega_{i}\right), i, j$, and $k$ are the labels for molecular coordinates $x, y$, and $z$, and the orientational average \langle\rangle$_{\text {iso }}$ is carried out for isotropic distribution. Experimentally, by using polarization combinations SPP (S-polarized $\omega_{s}, \mathrm{P}-$ polarized $\omega_{1}$, and P-polarized $\omega_{2}$ waves), PSP, and PPS, one can probe the value of $\chi_{\text {chiral }}^{(2)}$. 
(For the definition of $\mathrm{S}$ and $\mathrm{P}$ polarization, see Fig. 2.1(a).)

However, since in SFG we always measure $\left|\chi_{\text {eff }}^{(2)}\right|^{2}$, for a simple experiment where $\left|\chi_{\text {chiral }}^{(2)}\right|^{2}$ is measured, one only measures the amplitude of $\chi_{\text {chiral }}^{(2)}$. As a result, we can only determine whether the liquid is chiral, but cannot distinguish the enantiomers. We name this kind of measurement optically-active SFG (OA-SFG). Nevertheless, it is possible to distinguish the enantiomers. As shown in Chapter 4, we can distinguish the enantiomers and achieve chiral SFG measurement by deducing $\chi_{\text {chiral }}^{(2)}$ from interference measurements. In Chapter 6, by utilizing parity nonconservation, we are able to further devise a scheme using SFG to transmit chiral information via an achiral channel.

\subsubsection{Electronic resonance and vibrational resonance cases}

If sum frequency $\omega_{s}$ is in resonance with a transition from the electronic ground state $|g\rangle$ to the electronic excited state $|e\rangle$, as shown in Fig. 2.1(b), we have

$$
\chi_{\text {chiral }}^{(2)}=\frac{1}{\epsilon_{0}} \frac{N_{\mathrm{B}} l_{\mathrm{B}}\left(\omega_{s}\right) l_{\mathrm{B}}\left(\omega_{1}\right) l_{\mathrm{B}}\left(\omega_{2}\right)}{6 \hbar^{2}} \sum_{n} \frac{\left(\omega_{1}-\omega_{2}\right)}{\left(\omega_{s}-\omega_{n g}+i \Gamma_{n g}\right)} \sum_{n^{\prime}} \frac{\vec{\mu}_{g n} \cdot\left(\vec{\mu}_{n n^{\prime}} \times \vec{\mu}_{n^{\prime} g}\right)}{\left(\omega_{1}-\omega_{n^{\prime} g}\right)\left(\omega_{2}-\omega_{n^{\prime} g}\right)} .
$$

Here the two input frequencies are assumed to be far away from electronic resonances. If $\omega_{1}=\omega_{2}$, as in the case of second harmonic generation (SHG), $\chi_{\text {chiral }}^{(2)}$ vanishes, thus SHG is not allowed in chiral liquids.

If input beam $\omega_{2}$ is in resonance with a vibrational transition $|g, 0\rangle \rightarrow|g, 1\rangle$, we have

$$
\chi_{\text {chiral }}^{(2)}=\frac{1}{\epsilon_{0}} \frac{N_{\mathrm{B}} l_{\mathrm{B}}\left(\omega_{s}\right) l_{\mathrm{B}}\left(\omega_{1}\right) l_{\mathrm{B}}\left(\omega_{2}\right)}{6 \hbar} \frac{\vec{\mu}_{(g, 1)(g, 0)}}{\omega_{2}-\omega_{(g, 1)(g, 0)}+i \Gamma_{(g, 1)(g, 0)}} \cdot \vec{M}^{A}
$$


with

$$
\vec{M}^{A}=\frac{1}{\hbar} \sum_{n, \delta}\left[\frac{\vec{\mu}_{(g, 0)(n, \delta)} \times \vec{\mu}_{(n, \delta)(g, 1)}}{\omega_{s}-\omega_{(n, \delta)(g, 0)}+i \Gamma_{(n, \delta)(g, 0)}}+\frac{\vec{\mu}_{(g, 0)(n, \delta)} \times \vec{\mu}_{(n, \delta)(g, 1)}}{\omega_{s}-\omega_{(g, 1)(n, \delta)}+i \Gamma_{(g, 1)(n, \delta)}}\right]
$$

Here $|n, \delta\rangle$ denotes a vibronic state where $n$ labels the electronic state and $\delta$ the vibrational state. $\vec{M}^{A}$ is the antisymmetric part of the anti-Stokes Raman tensor [31].

Although the forms of $\chi_{\text {chiral }}^{(2)}$ appear different in Eqs. (2.15) and (2.16), they have some mutual features. They both show that $\chi_{\text {chiral }}^{(2)}$, as a pseudoscalar, changes sign under inversion. Therefore the $\chi_{\text {chiral }}^{(2)}$ 's of enantiomers have opposite signs, and racemic mixture has its $\chi_{\text {chiral }}^{(2)}$ equal to zero. This makes it a quantity sensitive to the presence of chirality [32]. Both equations show that $\chi_{\text {chiral }}^{(2)}$ is enhanced when their respective resonance conditions are satisfied. Thus by tuning the frequency of one of the input beams, one can obtain chiralitysensitive electronic and vibrational spectra.

The difference between the electronic resonance and vibrational resonance cases is that, in order for the vibrational resonance enhanced $\chi_{\text {chiral }}^{(2)}$ from chiral liquids to be nonzero, the anti-symmetric Raman coefficient $\vec{M}^{A}$ should not vanish. Corrections on Born-Oppenheimer approximation must be made so that $|n, \delta\rangle \neq|n\rangle|\delta\rangle$. In other words, only through vibronic coupling and nonadiabatic correction does the anti-symmetric Raman coefficient $\vec{M}^{A}$ become nonvanishing. When sum frequency is far from electronic resonances, corrections on the Born-Oppenheimer approximation are small, and it was found that the anti-symmetric Raman coefficient is two to three orders of magnitude less than its symmetric counterpart [31]. Since electronic resonance SFG does not have this limitation, vibrational resonance SFG from chiral liquids is generally weaker than the electronic one. 
However, if in the vibrational resonance case, sum frequency approaches an electronic transition, we have electronic-vibrational double-resonance sum-frequency generation. The double-resonance enhancement effect on chirality-sensitive SFG is more significant than the usual double-resonance enhancement in achiral systems. This is because the Born-Oppenheimer approximation becomes progressively worse as $\omega_{s}$ approaches electronic resonance. Therefore, in addition to the usual resonance enhancement, further enhancement of $\chi_{\text {chiral }}^{(2)}$ comes in through the corrections to the Born-Oppenheimer approximation. Near electronic resonances, the anti-symmetric and symmetric Raman coefficients become comparable [31]. As a result, chirality-sensitive SFG vibrational spectrum of a monolayer can be observed [19]. In this thesis, we focus on the electronic resonance cases. Vibrational resonance and electric-vibrational double-resonance OA-SFG have been reviewed elsewhere $[33,34]$.

\subsubsection{Comparison of SFG with conventional techniques}

One may ask why we need another technique to probe chirality when conventional techniques probing chirality, such as circular dichroism (CD), optical rotatory dispersion (ORD), vibrational circular dichroism (VCD), and Raman optical activity (ROA), are already well developed [3]. The reason lies in the sensitivity. The conventional techniques rely on the higher-order responses of the molecular system to the probing light. In other words, their responses would vanish if the interactions involved were only electric-dipole coupling between molecules and light. They become nonvanishing through the higher order interactions, such as the magnetic-dipole and electric-quadrupole couplings that are orders 


\subsection{SUM-FREQUENCY VIBRATIONAL SPECTROSCOPY (SFVS) OF} ACHIRAL INTERFACES

of magnitude weaker than the electric-dipole coupling. As a result, the sensitivity of these techniques, as determined by the ratio of the chiral response to the achiral response (from pure electric-dipole coupling), is usually not sufficient to detect chirality of monolayers and thin films.

In comparison, chirality-sensitive SFG in chiral liquids is allowed within electricdipole approximation, and $\chi_{\text {chiral }}^{(2)}$ can be selectively probed using polarization combinations SPP, PSP, and PPS without the electric-dipole allowed background contribution. Thus, whereas in conventional techniques a small difference between two large quantities (for the case of $\mathrm{CD}$ and ORD, $n_{\mathrm{L}}$ and $n_{\mathrm{R}}$, with $n$ being refractive index) needs to be measured, in SFG the existence of the signal alone is sufficient to ascertain that the sample is chiral. As a result, SFG has higher sensitivity and has been used to obtain optically active electronic and vibrational spectra of a monolayer of chiral molecules [19, 23]. SHG, although forbidden in chiral liquids, has been shown by Hicks et al. to be able to probe the chirality of a monolayer $[8,9,10,11,12]$.

\subsection{Sum-frequency vibrational spectroscopy (SFVS) of achi-}

\section{ral interfaces}

For a medium with inversion symmetry, at its surface the inversion symmetry is necessarily broken. Therefore, SFG is only allowed at its surface within the electricdipole approximation, thus is a surface-selective probe. ${ }^{1}$ By tuning the frequency of one

\footnotetext{
${ }^{1}$ Beyond the electric-dipole approximation, SFG is allowed in centrosymmetric systems if electric quadrupole and magnetic dipole contributions are considered. But the effect of these higher-order con-
} 


\subsection{SUM-FREQUENCY VIBRATIONAL SPECTROSCOPY (SFVS) OF} ACHIRAL INTERFACES
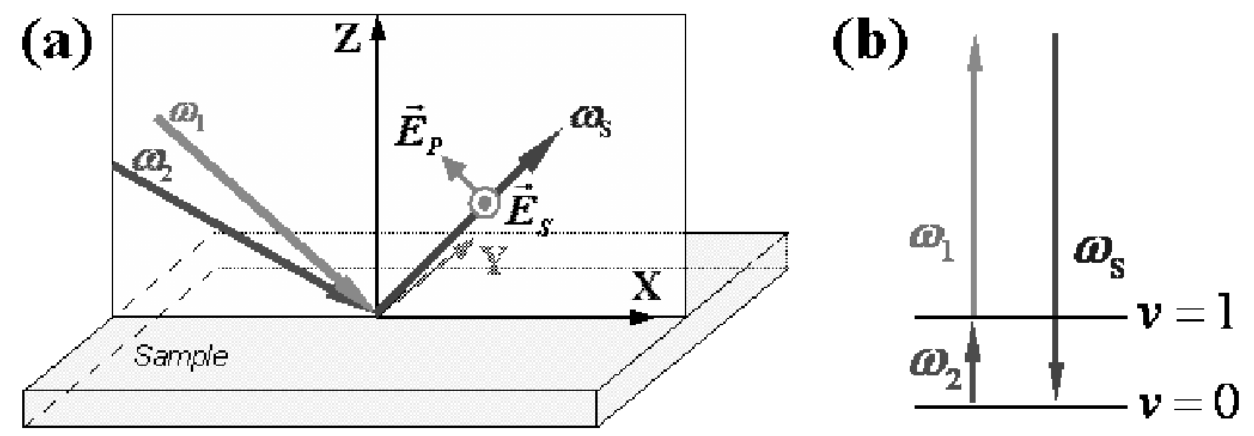

Figure 2.2: (a) Schematic view of the beam geometry at sample position and (b) energy level diagrams for typical sum-frequency vibrational spectroscopy (SFVS) experiments. Beam polarization is defined as $\mathrm{P}$ if the electric field vector $\vec{E}_{i}$ is parallel to the incidence plane, and $\mathrm{S}$ if $\vec{E}_{i}$ is perpendicular to the incidence plane.

input wave, we can obtain spectra of surface species through resonant enhancement. In particular, vibrational spectra of surface species are of most interest, because vibrational frequencies are sensitive to local environment. We name this type of measurement sumfrequency vibrational spectroscopy (SFVS). In a typical SFVS experiment, input beam $\omega_{1}$ is fixed at $532 \mathrm{~nm}$, while the other input beam $\left(\omega_{2}\right)$ is tuned across the vibrational resonances of the medium. This way, the generated sum-frequency photon is in the visible region and can be detected with high efficiency. The energy level diagram of SFVS is shown in Fig. 2.2(b).

For SFVS, Eq. (2.11) changes into

$$
\overleftrightarrow{\chi}^{(2)}=\overleftrightarrow{\chi}_{\mathrm{NR}}^{(2)}+\sum_{q} \frac{\overleftrightarrow{A}_{q}}{\omega_{2}-\omega_{q}+i \Gamma_{q}}
$$

where $q$ is the label of vibrational transitions, and the amplitude $\overleftrightarrow{A}_{q}$ is related to the tributions can be minimized by detecting the signal in the reflection direction [35]. 


\subsection{SUM-FREQUENCY VIBRATIONAL SPECTROSCOPY (SFVS) OF}

vibrational transition dipole moment derivative $\sqrt{\frac{\hbar}{2 \omega_{q}}} \frac{\partial \mu}{\partial Q_{q}}$ and Raman polarizability tensor

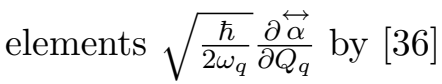

$$
A_{q, i j k}=-\frac{N_{\mathrm{S}}}{\epsilon_{0} \hbar} \sum_{i^{\prime} j^{\prime} k^{\prime}} \frac{\hbar}{2 \omega_{q}} \frac{\partial \alpha_{i^{\prime} j^{\prime}}}{\partial Q_{q}} \frac{\partial \mu_{k^{\prime}}}{\partial Q_{q}}\left\langle\left(i \cdot i^{\prime}\right)\left(j \cdot j^{\prime}\right)\left(k \cdot k^{\prime}\right)\right\rangle .
$$

Here $N_{\mathrm{S}}$ is the surface density of molecules, $i, j$, and $k$ refer to the laboratory coordinates, $i^{\prime}, j^{\prime}$, and $k^{\prime}$ to the molecular coordinates, $Q_{q}$ denotes the normal coordinate of vibrational mode $q$, and the angular brackets represent an average over molecular orientations.

Eq. (2.19) indicates that only vibrational modes that are both infrared and Raman active can be detected in SFVS. Since both the vibrational transition dipole moment and the Raman polarizability can be measured with other methods, by measuring the nonzero elements of $\overleftrightarrow{\chi}^{(2)}$, one can obtain both the density and the orientation of the surface species.

\subsubsection{Polarization combinations in SFVS}

In practice, to obtain the above information, one measures the SFVS spectra using different polarization combinations of the sum-frequency and the input waves. For an azimuthally isotropic surface, the second-order susceptibility tensor $\overleftrightarrow{\chi}_{\mathrm{S}}^{(2)}$ has 7 nonzero elements:

$$
\begin{aligned}
& \chi_{\mathrm{S}, \mathrm{XXZ}}^{(2)}=\chi_{\mathrm{S}, \mathrm{YYZ}}^{(2)} \\
& \chi_{\mathrm{S}, \mathrm{XZX}}^{(2)}=\chi_{\mathrm{S}, \mathrm{YZY}}^{(2)} \\
& \chi_{\mathrm{S}, \mathrm{ZXX}}^{(2)}=\chi_{\mathrm{S}, \mathrm{ZYY}}^{(2)} \\
& \chi_{\mathrm{S}, \mathrm{ZZZ}}^{(2)},
\end{aligned}
$$




\subsection{SUM-FREQUENCY VIBRATIONAL SPECTROSCOPY (SFVS) OF} ACHIRAL INTERFACES

where X, Y, Z are the laboratory coordinates defined previously and shown in Fig. 2.2(a). To deduce the value of the nonzero components, four polarization combinations are often needed: SSP, SPS, PSS, and PPP. The effective second-order nonlinear susceptibilities under these polarization combinations are

$$
\begin{aligned}
\chi_{\mathrm{eff}, \mathrm{SSP}}^{(2)}= & L_{\mathrm{YY}}\left(\omega_{s}\right) L_{\mathrm{YY}}\left(\omega_{1}\right) L_{\mathrm{ZZ}}\left(\omega_{2}\right) \sin \alpha_{1}\left(\omega_{2}\right) \chi_{\mathrm{S}, \mathrm{YYZ}}^{(2)} \\
\chi_{\mathrm{eff}, \mathrm{SPS}}^{(2)}= & L_{\mathrm{YY}}\left(\omega_{s}\right) L_{\mathrm{ZZ}}\left(\omega_{1}\right) L_{\mathrm{YY}}\left(\omega_{2}\right) \sin \alpha_{1}\left(\omega_{1}\right) \chi_{\mathrm{S}, \mathrm{YZY}}^{(2)} \\
\chi_{\mathrm{eff}, \mathrm{PSS}}^{(2)}= & L_{\mathrm{ZZ}}\left(\omega_{s}\right) L_{\mathrm{YY}}\left(\omega_{1}\right) L_{\mathrm{YY}}\left(\omega_{2}\right) \sin \alpha_{1}\left(\omega_{s}\right) \chi_{\mathrm{S}, \mathrm{ZYY}}^{(2)} \\
\chi_{\mathrm{eff}, \mathrm{PPP}}^{(2)}= & -L_{\mathrm{XX}}\left(\omega_{s}\right) L_{\mathrm{XX}}\left(\omega_{1}\right) L_{\mathrm{ZZ}}\left(\omega_{2}\right) \cos \alpha_{1}\left(\omega_{s}\right) \cos \alpha_{1}\left(\omega_{1}\right) \sin \alpha_{1}\left(\omega_{2}\right) \chi_{\mathrm{S}, \mathrm{XXZ}}^{(2)} \\
& -L_{\mathrm{XX}}\left(\omega_{s}\right) L_{\mathrm{ZZ}}\left(\omega_{1}\right) L_{\mathrm{XX}}\left(\omega_{2}\right) \cos \alpha_{1}\left(\omega_{s}\right) \sin \alpha_{1}\left(\omega_{1}\right) \cos \alpha_{1}\left(\omega_{2}\right) \chi_{\mathrm{S}, \mathrm{XZX}}^{(2)} \\
& +L_{\mathrm{ZZ}}\left(\omega_{s}\right) L_{\mathrm{XX}}\left(\omega_{1}\right) L_{\mathrm{XX}}\left(\omega_{2}\right) \sin \alpha_{1}\left(\omega_{s}\right) \cos \alpha_{1}\left(\omega_{1}\right) \cos \alpha_{1}\left(\omega_{2}\right) \chi_{\mathrm{S}, \mathrm{ZXX}}^{(2)} \\
& +L_{\mathrm{ZZ}}\left(\omega_{s}\right) L_{\mathrm{ZZ}}\left(\omega_{1}\right) L_{\mathrm{ZZ}}\left(\omega_{2}\right) \sin \alpha_{1}\left(\omega_{s}\right) \sin \alpha_{1}\left(\omega_{1}\right) \sin \alpha_{1}\left(\omega_{2}\right) \chi_{\mathrm{S}, \mathrm{ZZZ}}^{(2)},
\end{aligned}
$$

where $\alpha_{1}\left(\omega_{i}\right)$ is the incident angle of wave $\omega_{i}$ in the air. Because the anti-symmetric part of the Raman tensor away from electronic resonances is often small [31],

$$
\chi_{\mathrm{S}, \mathrm{XZX}}^{(2)}=\chi_{\mathrm{S}, \mathrm{YZY}}^{(2)}=\chi_{\mathrm{S}, \mathrm{ZXX}}^{(2)}=\chi_{\mathrm{S}, \mathrm{ZYY}}^{(2)}
$$

Under this condition, only SSP, SPS (or PSS), and PPP spectra are needed. For an anisotropic surface, more measurements are needed.

In this thesis, we show how SFVS can be used to obtain the surface density and orientation of adsorbed leucine molecules at air-water interface in Chapter 7. In Chapter 8, we use SFVS to study shear-aligned poly(tetrafluoroethylene) film, where the selection rule of SFVS is used to assign the symmetry of normal modes of poly(tetrafluoroethylene). 


\subsection{Experimental setup}

As a nonlinear optical process, SFG is a weak effect and needs the input waves to have high intensity. In most of the work described in this thesis, a pulsed picosecond laser system that generates coherent radiation with either fixed or tunable frequencies was used.

Briefly, we employed a high power picosecond Nd:YAG laser (Continuum Inc.) operated at $20 \mathrm{~Hz}$, and a homemade laser-pumped optical parametric generator/amplifier (OPG/OPA) system [37] generating tunable coherent output from $230 \mathrm{~nm}$ to $10 \mu \mathrm{m}$, with a $\sim 20$ ps pulse width. Two noncollinear beams of different frequencies were then overlapped spatially and temporally at the sample. Sum-frequency output is allowed in both transmission and reflection directions as defined by photon momentum conservation [1]. For measurements of achiral surfaces, the input beams were overlapped at the surface of interest and the sum-frequency output was detected in the reflection direction to minimize the surface-insensitive contribution due to the electric quadrupole and magnetic dipole contributions [35]. For chirality-sensitive SFG measurement from chiral liquids, sum-frequency signals were detected in the transmission direction to take advantage of the longer coherent length. The beams were overlapped at the bottom liquid-cell interface for electronic resonance SFG, for reasons explained in Appendix A.

Being spectrally distinct and spatially directional, sum-frequency signals can be detected without much background after proper spectral and spatial filtering. The detection system used was a Hamamatsu photomultiplier tube in connection with a Stanford Research SR250 gated integrator. For vibrational resonance studies, a $532 \mathrm{~nm}$ beam $\left(\omega_{1}\right)$ and a tunable 
IR beam $\left(\omega_{2}\right)$ were used as inputs. The typical power of the visible beam was on the order of a few mJ per pulse, and that of IR beam on the order of tens to hundreds of $\mu \mathrm{J}$. For chirality-sensitive SFG studies of electronic transitions, a tunable UV/visible beam from the OPG/OPA $\left(\omega_{1}\right)$ (hundreds of $\mu \mathrm{J}$ per pulse) and a 1064nm beam $\left(\omega_{2}\right)(2 \mathrm{~mJ}$ per pulse) from the Nd:YAG laser were the inputs. By varying $\omega_{1}$, sum frequency $\omega_{s}$ was tuned over the electronic transitions of the sample. The detected sum-frequency signal varied from $\sim 0.01$ to $\sim 10$ photons per pulse, depending on the experiment geometry, the sample, and its concentration. Accumulation of tens of minutes is needed to obtain a spectrum with the typical signal-to-noise ratio of the spectra shown in this thesis, whereas measurements at a single wavelength to determine the presence of a nonzero signal take only seconds, and can be further sped up by using lasers of higher repetition rates. 
Chapter 3

A dynamic coupling model for

sum-frequency chiral response

from liquids composed of molecules

with a chiral side chain and an

achiral chromophore

3.1 Introduction

Chirality is the property that an object cannot be superimposed by translation and rotation on its mirror image. In terms of symmetry, a chiral object does not have sym- 
metry planes, inversion centers or improper rotation axes. Chirality is a common property of objects in nature, with most natural biological molecules, from amino acids to hormones, being chiral. The study on molecular chirality has a history as long as that of modern chemistry itself. Since the early eighteenth century, optical properties have been used to study molecular chirality [38]. The most commonly employed one is linear optical activity such as optical rotatory dispersion (ORD) and circular dichroism (CD), which result from chiral media having different refractive indices for left- and right-circularly polarized light $[39,40]$. Rosenfeld was the first to give a quantum mechanical description for linear optical activity [41]. He pointed out that the strength of ORD and CD near an electronic transition $|g\rangle \rightarrow|n\rangle$ depends on the rotatory strength $R_{g n}$, with

$$
R_{g n}=\operatorname{Im}\left\{\vec{\mu}_{g n} \cdot \vec{m}_{n g}\right\}
$$

where $\vec{\mu}$ and $\vec{m}$ are the electric and magnetic dipole transition moments between states $|g\rangle$ and $|n\rangle$, respectively. Because the magnitude and directions of the dipole transition moments are determined by wave functions of the electronic states $|g\rangle$ and $|n\rangle$, the rotatory strength $R_{g n}$ is related to the structural characteristics of the molecule. It can be shown that $R_{g n}$ has opposite signs for the two enantiomers of a chiral molecule, and is zero for achiral molecules. This ability of detecting molecular chirality has made CD an important technique in fields ranging from organic structural chemistry to structural biology.

It was proposed by Giordmaine in 1965 that second-order nonlinear optical effects such as sum-frequency generation (SFG) can also be used to detect chirality in optically active liquids [16]. However, it was not until 2000 that this effect was observed unequivo- 
cally [17]. Since then, optically active sum-frequency generation (OA-SFG) spectra of vibrational, electronic and vibrational-electronic double resonances of various chiral molecules in solutions have been obtained $[18,19,20,21]$. In OA-SFG, the chiral response is allowed in liquids under electric dipole approximation. Consequently, it has a much higher sensitivity than $\mathrm{CD}$, where a magnetic dipole transition must be involved. Demonstrations of high sensitivity of OA-SFG can be found in literature: optically active electronic and vibrational spectra of monolayers of chiral molecules were observed $[19,23]$. (Second harmonic generation circular dichroism, which is forbidden in liquids but allowed at interfaces, was also observed from chiral monolayers in 1993 by Hicks et al [8].) This opens up the exciting possibility of using OA-SFG to monitor important biological and chemical processes involving chiral molecules that cannot be observed with conventional methods such as CD.

In order to use second-order nonlinear chiral optical processes to obtain information such as molecular configuration or conformation, we have to understand how the chiral responses are related to the molecular structure. As intrinsically different processes, electronically resonant OA-SFG and CD provide different information on the chiral molecular structure. For molecules composed of a set of twisted dimers such as binaphthal, a coupled-oscillator theory was proposed to describe the electronically resonant OA-SFG, and comparison with the coupled-oscillator theory for CD was made [42]. Most biological molecules, however, are composed of an intrinsically achiral chromophore and a chiral center, as do many pharmaceutical compounds. Given their importance, we need to understand the mechanism of chirality-sensitive SFG from these molecules near electronic resonances of their achiral chromophores. In this chapter, we describe our effort in applying OA-SFG 


\subsection{THEORETICAL BASIS OF SFG FROM ISOTROPIC CHIRAL} LIQUIDS

to this class of chiral molecules. We use amino acids as model systems and develop a general theory for electronically resonant OA-SFG from solutions of molecules made of an intrinsically achiral chromophore and a chiral side chain.

This chapter is organized as following: first, the general theory on OA-SFG from an isotropic solution of chiral molecules with a chiral center is outlined, followed by an electron correlation model first developed by Höhn and Weigang [43], from which a dynamic coupling model for OA-SFG is presented. Using amino acids as model systems, we then describe the formulism to evaluate the strength of the optically active second-order nonlinear optical effect. In particular, we focus on cases where the chiral side chain is made of nonpolar bonds. We further compare the experimental results with those predicted by our model. In the subsequent discussion, we propose a few tentative rules on the effectiveness of the chiralityinducing perturbations on achiral chromophores. Sum-frequency spectra from several amino acids with polar or charged side chains are also presented and discussed.

\subsection{Theoretical basis of SFG from isotropic chiral liquids}

For chiral liquids, their OA-SFG signal is governed by the second order nonlinear susceptibility $\chi_{\text {chiral }}^{(2)}$, which is

$$
\chi_{\text {chiral }}^{(2)} \equiv \chi_{\mathrm{XYZ}}^{(2)}=-\chi_{\mathrm{XZY}}^{(2)}=\chi_{\mathrm{YZX}}^{(2)}=-\chi_{\mathrm{YXZ}}^{(2)}=\chi_{\mathrm{ZXY}}^{(2)}=-\chi_{\mathrm{ZYX}}^{(2)}
$$

where $\mathrm{X}, \mathrm{Y}$ and $\mathrm{Z}$ are the laboratory coordinate axes, and $\chi_{\text {chiral }}^{(2)}$ is of opposite signs for the two enantiomers and zero for a racemic mixture. The OA-SFG signal is proportional to $\left|\chi_{\text {chiral }}^{(2)}\right|^{2}$, and can be detected when polarization combinations SPP, PSP, and PPS are 


\subsection{THEORETICAL BASIS OF SFG FROM ISOTROPIC CHIRAL LIQUIDS}

used.

Microscopically, $\chi_{\text {chiral }}^{(2)}$ is determined by the chiral molecular structure by

$$
\chi_{\text {chiral }}^{(2)} \propto N \frac{1}{6 \hbar^{2} \epsilon_{0}} \sum_{n} \frac{\left(\omega_{1}-\omega_{2}\right)}{\left(\omega_{s}-\omega_{n g}+i \Gamma_{n g}\right)} \sum_{n^{\prime}} \frac{\vec{\mu}_{g n} \cdot\left(\vec{\mu}_{n n^{\prime}} \times \vec{\mu}_{n^{\prime} g}\right)}{\left(\omega_{1}-\omega_{n^{\prime} g}\right)\left(\omega_{2}-\omega_{n^{\prime} g}\right)},
$$

where $N$ is the number density of the molecule, and $\omega_{i j}, \Gamma_{i j}$, and $\vec{\mu}_{i j}$ the transition frequency, the damping constant, and the electric-dipole matrix element of the transition between electronic states $|i\rangle$ and $|j\rangle$. Here the two input frequencies are assumed to be far away from electronic resonances. As seen from Eq. (3.3), scanning $\omega_{s}$ over an electronic transition by tuning one of the input beams yields an optically active electronic spectrum.

An important conclusion from Eq. (3.3) is that in order for OA-SFG to be allowed, the three electric-dipole transition moments $\vec{\mu}_{g n}, \vec{\mu}_{n n^{\prime}}$, and $\vec{\mu}_{n^{\prime} g}$ must not be coplanar. Intrinsically achiral chromophores have either a center of inversion or at least one plane of symmetry, so that the above condition cannot be satisfied, and hence $\chi_{\text {chiral }}^{(2)}$ is always zero. When the extrachromophoric molecular structures are chiral, their interactions with the chromophore lower the symmetry of its electronic states, making the above condition satisfied. In the language of perturbation theory, the extrachromophoric perturbations change the wavefunctions of the chromophore, and make the three transition moments nonplanar.

In principle, the magnitude and directions of electric-dipole transition moments could be obtained by ab initio calculation. In reality, however, it is a demanding task, for potential energy surfaces of excited electronic states need to be calculated with high precision. Furthermore, many important chiral systems, in particular the biologically important 
ones, are in aqueous solutions with the presence of ions. With the specific interactions between solvent and solute molecules taken into account, the calculation becomes very expensive even for molecules with moderate sizes [44]. An analytic method based on physical pictures is often more helpful to achieve an understanding of the origin of OA-SFG.

Fortunately, the problem of calculating transition moments that are revised by chiral perturbations is not a new one. In linear optical activity, the rotatory strength is related to the product of electric-dipole and magnetic-dipole transition moments as shown in Eq. (3.1). For an achiral chromophore, the two moments are either forbidden or perpendicular to each other. The nonzero CD and ORD signals near an electronic transition result from chiral perturbations, which make the transition moments nonzero and not perpendicular to each other. Many different theoretical approaches for such a perturbation calculation were developed [39]. In the following sections, we will show that the electron correlation model, originally proposed by Höhn and Weigang to calculate the rotatory strength of linear optical activity [43], can be directly adapted to calculate the nonlinear optical response in OA-SFG.

\subsection{Electron correlation model}

In calculating the perturbed electric-dipole transition moments, a chiral molecule composed of an achiral chromophore and a chiral molecular surrounding may be divided in the zeroth order into two electronically independent groups with negligible interaction. Then, for the molecular state with the chromophore (A) in its $m$ th eigenstate and the sur- 
rounding perturber (B) in its $n$th eigenstate, the wave function is $\left.\left.\left.\mid A_{m} B_{n}\right)=\mid A_{m}\right) \mid B_{n}\right)$. In the first-order correction, with the interaction $V$ between the chromophore and its surrounding taken into account, the wavefunction becomes [43]

$$
\left.\left.\left|A_{m} B_{l}\right\rangle=\mid A_{m} B_{l}\right)+\sum_{i} \sum_{j} \frac{\left(A_{i} B_{j}|V| A_{m} B_{l}\right)}{E_{m}^{\mathrm{A}}+E_{l}^{\mathrm{B}}-E_{i}^{\mathrm{A}}-E_{j}^{\mathrm{B}}} \mid A_{i} B_{j}\right),
$$

within the framework of nondegenerate perturbation theory. The electric-dipole transition moment between states $\left|A_{m} B_{0}\right\rangle$ and $\left|A_{n} B_{0}\right\rangle$ is thus

$$
\begin{aligned}
\left\langle A_{n} B_{0}|\vec{\mu}| A_{m} B_{0}\right\rangle & =\left(A_{n} B_{0}|\vec{\mu}| A_{m} B_{0}\right) \\
& +\sum_{i \neq m} \frac{\left(A_{i} B_{0}|V| A_{m} B_{0}\right)}{E_{m}^{\mathrm{A}}-E_{i}^{\mathrm{A}}}\left(A_{n} B_{0}|\vec{\mu}| A_{i} B_{0}\right) \\
& +\sum_{j \neq n} \frac{\left(A_{n} B_{0}|V| A_{j} B_{0}\right)}{E_{n}^{\mathrm{A}}-E_{j}^{\mathrm{A}}}\left(A_{j} B_{0}|\vec{\mu}| A_{m} B_{0}\right) \\
& +\sum_{l \neq 0} \frac{\left(A_{n} B_{l}|V| A_{m} B_{0}\right)}{E_{m}^{\mathrm{A}}-E_{n}^{\mathrm{A}}-E_{l}^{\mathrm{B}}}\left(A_{n} B_{0}|\vec{\mu}| A_{n} B_{l}\right) \\
& +\sum_{l \neq 0} \frac{\left(A_{n} B_{0}|V| A_{m} B_{0}\right)}{E_{n}^{\mathrm{A}}-E_{m}^{\mathrm{A}}-E_{l}^{\mathrm{B}}}\left(A_{m} B_{l}|\vec{\mu}| A_{m} B_{0}\right) .
\end{aligned}
$$

Because

$$
\vec{\mu}=\vec{\mu}_{\mathrm{A}}+\vec{\mu}_{\mathrm{B}}
$$


Eq. (3.5) becomes

$$
\begin{aligned}
\left\langle A_{n} B_{0}|\vec{\mu}| A_{m} B_{0}\right\rangle & =\left(A_{n}\left|\vec{\mu}_{A}\right| A_{m}\right) \\
& +\sum_{i \neq m} \frac{\left(A_{i} B_{0}|V| A_{m} B_{0}\right)}{E_{m}^{\mathrm{A}}-E_{i}^{\mathrm{A}}}\left(A_{n}\left|\vec{\mu}_{\mathrm{A}}\right| A_{i}\right) \\
& +\sum_{j \neq n} \frac{\left(A_{n} B_{0}|V| A_{j} B_{0}\right)}{E_{n}^{\mathrm{A}}-E_{j}^{\mathrm{A}}}\left(A_{j}\left|\vec{\mu}_{\mathrm{A}}\right| A_{m}\right) \\
& +\sum_{l \neq 0} \frac{\left(A_{n} B_{l}|V| A_{m} B_{0}\right)}{E_{m}^{\mathrm{A}}-E_{n}^{\mathrm{A}}-E_{l}^{\mathrm{B}}}\left(B_{0}\left|\vec{\mu}_{\mathrm{B}}\right| B_{l}\right) \\
& +\sum_{l \neq 0} \frac{\left(A_{n} B_{0}|V| A_{m} B_{0}\right)}{E_{n}^{\mathrm{A}}-E_{m}^{\mathrm{A}}-E_{l}^{\mathrm{B}}}\left(B_{l}\left|\vec{\mu}_{\mathrm{B}}\right| B_{0}\right)
\end{aligned}
$$

where term (a) is the unperturbed electric-dipole transition moment of the chromophore, terms (b) and (c) are moments "borrowed" from the other states $\left.\mid A_{l}\right)(l \neq m, n)$ of the chromophore, terms (d) and (e) are borrowed from the states of the perturber. The strength borrowed is related to the perturbation matrix elements of $V$.

The interaction potential $V$ between the chromophore and the perturber is Coulombic in nature, and can be expanded into static multipole-multipole interactions, as tabulated in the original paper by Höhn and Weigang [43]. For an electric-dipole forbidden transition, $\left(A_{n}\left|\vec{\mu}_{\mathrm{A}}\right| A_{m}\right)=0$. Furthermore, if the perturber is neither charged nor polar such that $\left(B_{0}|V| B_{0}\right)=0$, the leading interaction is the quadrupole-dipole term in $V$, and (d) and (e) are the only nonvanishing terms in the correction. Only when the perturber is polar or charged, will terms (b) and (c) contribute. Because (b) and (c) originate from the static charges and multipolar fields of the perturber, they are historically named as the static coupling terms, whereas (d) and (e) are referred to as the dynamic coupling terms due to the correlative electronic interactions between the chromophore and the perturber through 


\subsection{DYNAMIC COUPLING MODEL WITH AMINO ACIDS AS A MODEL SYSTEM}

$\left(A_{n} B_{l}|V| A_{m} B_{0}\right)$ and $\left(A_{n} B_{0}|V| A_{m} B_{l}\right)$.

With Eq. (3.7), the perturbed electric-dipole transition moments in OA-SFG can be evaluated. The calculation can often be simplified by symmetry arguments applied to the chromophore and the perturber. As an example, we apply this electron correlation model to a typical chiral system made of an achiral chromophore and a chiral perturber - amino acids. In particular, we shall focus on amino acids with nonpolar saturated side chains, for which only the dynamic coupling terms need to be considered in describing OA-SFG.

\subsection{Dynamic coupling model with amino acids as a model system}

Naturally occurred amino acids have the carboxyl group as their achiral chromophore, which is connected to an amide group, a hydrogen atom and a side chain through a carbon atom that is the chiral center. In alkaline solutions, the carboxyl group is deprotonated into a carboxylate anion $\left(-\mathrm{COO}^{-}\right)$with $C_{2 v}$ symmetry. We define the molecular coordinate $\hat{z}$ to be along the $C_{2}$ axis of $-\mathrm{COO}^{-}, \hat{x}$ perpendicular to the $-\mathrm{COO}^{-}$plane, and the origin at the center of gravity of $-\mathrm{COO}^{-}$charge distribution.

As discussed in the previous section, an amino acid molecule can be divided into two parts: $\mathrm{COO}^{-}$as the achiral chromophore and the rest as the chiral perturber. In the following, we show that the symmetry property of $-\mathrm{COO}^{-}$group allows us to obtain an expression, from which we can have a semi-quantitative estimation on the OA-SFG response

and compare it with the experimental result. We focus on the magnitude of $\chi_{\text {chiral }}^{(2)}$ as the 


\subsection{DYNAMIC COUPLING MODEL WITH AMINO ACIDS AS A

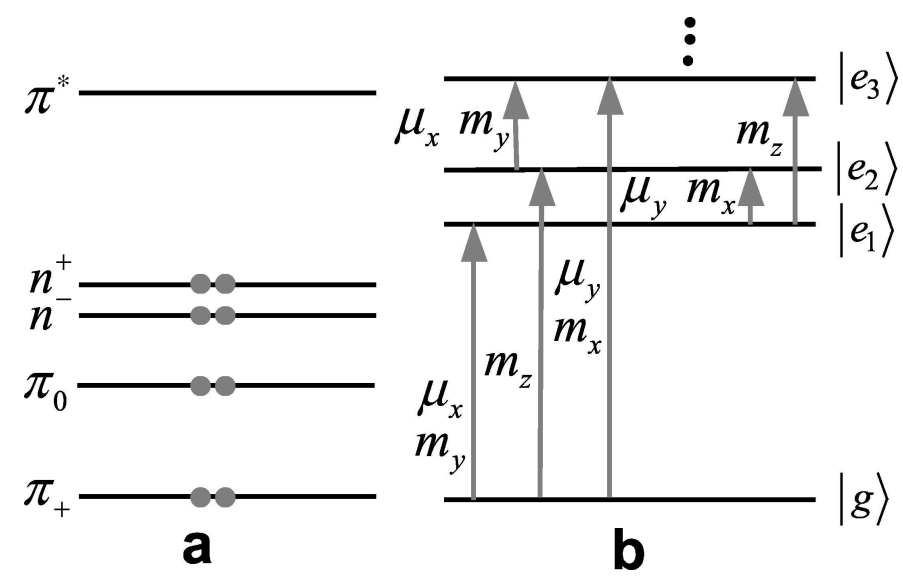

Figure 3.1: (a) Molecular orbitals of $-\mathrm{COO}^{-}$: dots are electrons arranged in the electron configuration of the ground electronic state $|g\rangle$. (b) Energy level diagram of -COO ${ }^{-}$group: ground state $|g\rangle$ and the first three excited states $\left|e_{1}\right\rangle,\left|e_{2}\right\rangle$, and $\left|e_{3}\right\rangle$. The arrows denote transitions between states, to the left of which are the allowed electric-dipole and magneticdipole transition moments.

sum frequency approaches the first electronic resonances of $-\mathrm{COO}^{-}$around $200 \mathrm{~nm}$.

We begin with the expression of $\chi_{\text {chiral }}^{(2)}$ for the unperturbed chromophore. From the molecular orbital theory, the ground electronic state $|g\rangle$ of $-\mathrm{COO}^{-}$has an electron configuration with the highest occupied molecular orbitals being $\pi_{+}, \pi_{0}, n^{-}$, and $n^{+}$, and the lowest unoccupied molecular orbital being the antibonding $\pi^{*}$, as shown in Fig. 3.1(a). If the configuration interaction is neglected, the lowest excited electronic states $\left|e_{1}\right\rangle,\left|e_{2}\right\rangle$ and $\left|e_{3}\right\rangle$ can be constructed by promoting one electron from $n^{+}, n^{-}$, and $\pi_{0}$ to $\pi^{*}$, respectively, as schematically shown in Fig. 3.1b. (From group theory, electronic states $\left.|g\rangle,\left|e_{1}\right\rangle, e_{2}\right\rangle$, and $\left.e_{3}\right\rangle$ have $A_{1}, B_{1}, A_{2}$, and $B_{2}$ symmetry, respectively.) The transitions from $|g\rangle$ to $\left.e_{1}\right\rangle$ and $\left.e_{2}\right\rangle$ are in the $180 \mathrm{~nm}$ to $210 \mathrm{~nm}$ region, and the $|g\rangle$ to $\left.e_{3}\right\rangle$ transition around $160 \mathrm{~nm}$ [45]. 


\subsection{DYNAMIC COUPLING MODEL WITH AMINO ACIDS AS A MODEL SYSTEM}

A more precise calculation on $\chi_{\text {chiral }}^{(2)}$ would need the inclusion of many excited states. However, with the next higher state around 110nm (corresponding to promoting an electron from $\pi_{+}$to $\pi^{*}$ ) [46], for $\chi_{\text {chiral }}^{(2)}$ near $200 \mathrm{~nm}$, a four-state model involving only states $|g\rangle,\left|e_{1}\right\rangle,\left|e_{2}\right\rangle$, and $\left|e_{3}\right\rangle$ should suffice to give a quantitative estimation.

Now, for this four-level system, we obtain from Eq. (3.3)

$$
\begin{aligned}
\chi_{\text {chiral }}^{(2)} & \propto \frac{1}{\omega_{s}-\omega_{e_{1} g}+i \Gamma_{e_{1} g}}\left[\frac{\vec{\mu}_{g e_{1}} \cdot\left(\vec{\mu}_{e_{1} e_{2}} \times \vec{\mu}_{e_{2} g}\right)}{\left(\omega_{1}-\omega_{e_{2} g}\right)\left(\omega_{2}-\omega_{e_{2} g}\right)}+\frac{\vec{\mu}_{g e_{1}} \cdot\left(\vec{\mu}_{e_{1} e_{3}} \times \vec{\mu}_{e_{3} g}\right)}{\left(\omega_{1}-\omega_{e_{3} g}\right)\left(\omega_{2}-\omega_{e_{3} g}\right)}\right] \\
& +\frac{1}{\omega_{s}-\omega_{e_{2} g}+i \Gamma_{e_{2} g}}\left[\frac{\vec{\mu}_{g e_{2}} \cdot\left(\vec{\mu}_{e_{2} e_{1}} \times \vec{\mu}_{e_{1} g}\right)}{\left(\omega_{1}-\omega_{e_{1} g}\right)\left(\omega_{2}-\omega_{e_{1} g}\right)}+\frac{\vec{\mu}_{g e_{2}} \cdot\left(\vec{\mu}_{e_{2} e_{3}} \times \vec{\mu}_{e_{3} g}\right)}{\left(\omega_{1}-\omega_{e_{3} g}\right)\left(\omega_{2}-\omega_{e_{3} g}\right)}\right] \\
& +\frac{1}{\omega_{s}-\omega_{e_{3} g}+i \Gamma_{e_{3} g}}\left[\frac{\vec{\mu}_{g e_{3}} \cdot\left(\vec{\mu}_{e_{3} e_{1}} \times \vec{\mu}_{e_{1} g}\right)}{\left(\omega_{1}-\omega_{e_{1} g}\right)\left(\omega_{2}-\omega_{e_{1} g}\right)}+\frac{\vec{\mu}_{g e_{3}} \cdot\left(\vec{\mu}_{e_{3} e_{2}} \times \vec{\mu}_{e_{2} g}\right)}{\left(\omega_{1}-\omega_{e_{2} g}\right)\left(\omega_{2}-\omega_{e_{2} g}\right)}\right]
\end{aligned}
$$

where $\vec{\mu}_{i j} \equiv\langle i|\vec{\mu}| j\rangle$. As shown in Fig. 3.1(b), the $-\mathrm{COO}^{-}$chromophore has only four nonvanishing electric-dipole transition matrix elements: $\vec{\mu}_{g e_{1}}$ and $\vec{\mu}_{e_{2} e_{3}}$ along $\hat{x}$, and $\vec{\mu}_{e_{1} e_{2}}$ and $\vec{\mu}_{g e_{3}}$ along $\hat{y}$, where the $-\mathrm{COO}^{-}$plane lies in $\hat{y}-\hat{z}$ plane and the symmetric axis of $-\mathrm{COO}^{-}$is along $\hat{z}$. Thus for the unperturbed $-\mathrm{COO}^{-}$, we have $\chi_{\text {chiral }}^{(2)}=0$ as expected. The first-order perturbation from the perturber makes $\vec{\mu}_{e_{2} g}$ and $\vec{\mu}_{e_{3} e_{1}}$ nonvanishing, and hence puts $\chi_{\text {chiral }}^{(2)}$ in the form

$$
\chi_{\text {chiral }}^{(2)} \propto f(\omega) \hat{x} \cdot\left(\hat{y} \times \vec{\mu}_{e_{2} g}\right)+g(\omega) \hat{x} \cdot\left(\hat{y} \times \vec{\mu}_{e_{3} e_{1}}\right) .
$$

As discussed in the previous section, because the perturber is neither charged nor polar, only the dynamic coupling terms appear, with quadrupole (chromophore)-dipole 


\subsection{DYNAMIC COUPLING MODEL WITH AMINO ACIDS AS A} MODEL SYSTEM

(perturber) interaction as the leading interaction:

$$
\begin{aligned}
V & =\frac{3}{2} R^{-7}\left\{\left[\left(3 R^{2} X-5 X^{3}\right) \mu_{x}(\mathrm{~B})+\left(R^{2} Y-5 X^{2} Y\right) \mu_{y}(\mathrm{~B})+\left(R^{2} Z-5 X^{2} Z\right) \mu_{z}(\mathrm{~B})\right] \Theta_{x x}(\mathrm{~A})\right. \\
& +\left[\left(R^{2} X-5 X Y^{2}\right) \mu_{x}(\mathrm{~B})+\left(3 R^{2} Y-5 Y^{3}\right) \mu_{y}(\mathrm{~B})+\left(R^{2} Z-5 Y^{2} Z\right) \mu_{z}(\mathrm{~B})\right] \Theta_{y y}(\mathrm{~A}) \\
& +\left[\left(R^{2} X-5 X Z^{2}\right) \mu_{x}(\mathrm{~B})+\left(R^{2} Y-5 Y Z^{2}\right) \mu_{y}(\mathrm{~B})+\left(3 R^{2} Z-5 Z^{3}\right) \mu_{z}(\mathrm{~B})\right] \Theta_{z z}(\mathrm{~A}) \\
& +2\left[\left(R^{2} Y-5 X^{2} Y\right) \mu_{x}(\mathrm{~B})+\left(R^{2} X-5 X Y^{2}\right) \mu_{y}(\mathrm{~B})-5 X Y Z \mu_{z}(\mathrm{~B})\right] \Theta_{x y}(\mathrm{~A}) \\
& +2\left[\left(R^{2} Z-5 X^{2} Z\right) \mu_{x}(\mathrm{~B})-5 X Y Z \mu_{y}(\mathrm{~B})+\left(R^{2} X-5 X Z^{2}\right) \mu_{z}(\mathrm{~B})\right] \Theta_{x z}(\mathrm{~A}) \\
& \left.+2\left[-5 X Y Z \mu_{x}(\mathrm{~B})+\left(R^{2} Z-5 Y^{2} Z\right) \mu_{y}(\mathrm{~B})+\left(R^{2} Y-5 Y Z^{2}\right) \mu_{z}(\mathrm{~B})\right] \Theta_{y z}(\mathrm{~A})\right\} .
\end{aligned}
$$

Here $R$ is the magnitude of $\vec{R}$ that describes the position of the center of gravity of the perturber's charge distribution with respect to the center of gravity of the chromophore's charge distribution, $X, Y$, and $Z$ are the components of $\vec{R}$ along the molecular coordinates of the chromophore, $\mu_{i}(\mathrm{~B})$ is the dipole operator of the perturber along the $i$ th molecular coordinate axis of the chromophore, and $\Theta_{i j}(\mathrm{~A})$ is the $(i j)$ tensor component of the quadrupole transition moment operator of the chromophore. With $-\mathrm{COO}^{-}$in the $\hat{y}-\hat{z}$ plane being of $C_{2 v}$ symmetry, only $\Theta_{x y}(\mathrm{~A})$ can contribute to the generation of $\vec{\mu}_{e_{2} g}$ and $\vec{\mu}_{e_{3} e_{1}}$. Equation (3.7) gives for the electric-dipole transition moments of the chromophore

$$
\begin{gathered}
\vec{\mu}_{e_{2} g}=\sum_{l \neq 0} \frac{6 E_{l}^{B} R^{-7}}{\left(E_{e_{2}}^{\mathrm{A}}-E_{g}^{\mathrm{A}}\right)^{2}-E_{l}^{\mathrm{B}^{2}}}\left[\left(R^{2} Y-5 X^{2} Y\right) \mu_{x}^{l 0}(\mathrm{~B})\right. \\
\left.+\left(R^{2} X-5 X Y^{2}\right) \mu_{y}^{l 0}(\mathrm{~B})-5 X Y Z \mu_{z}^{l 0}(\mathrm{~B})\right] \vec{\mu}^{0 l}(\mathrm{~B}) \Theta_{x y}^{e_{2} g}(\mathrm{~A}) \\
\vec{\mu}_{e_{3} e_{1}}=\sum_{l \neq 0} \frac{6 E_{l}^{B} R^{-7}}{\left(E_{e_{3}}^{\mathrm{A}}-E_{e_{1}}^{\mathrm{A}}\right)^{2}-E_{l}^{\mathrm{B}^{2}}}\left[\left(R^{2} Y-5 X^{2} Y\right) \mu_{x}^{l 0}(\mathrm{~B})\right. \\
\left.+\left(R^{2} X-5 X Y^{2}\right) \mu_{y}^{l 0}(\mathrm{~B})-5 X Y Z \mu_{z}^{l 0}(\mathrm{~B})\right] \vec{\mu}^{0 l}(\mathrm{~B}) \Theta_{x y}^{e_{3} e_{1}}(\mathrm{~A})
\end{gathered}
$$

It is noted from Eq. (3.9) that for OA-SFG of amino acids, the $z$ components of perturbation- 


\subsection{DYNAMIC COUPLING MODEL WITH AMINO ACIDS AS A MODEL SYSTEM}

induced $\vec{\mu}_{e_{2} g}$ and $\vec{\mu}_{e_{3} e_{1}}$ are responsible for the nonvanishing $\chi_{\text {chiral }}^{(2)}$. We therefore focus on these components in the following derivation.

Following Höhn and Weigang's treatment, we assume that the perturber has cylindrical symmetry and define its cylindrical axis as $\hat{z}^{\prime}$, which is oriented at a polar angle $\theta$ and an azimuthal angle $\phi$ with respect to the molecular coordinates $\hat{z}$ and $\hat{x}$ of the chromophore. Using Kirkwood's definition for polarizability [47]

$$
\overleftrightarrow{\alpha}(\nu)=\frac{2}{h} \sum_{n \neq 0} \frac{\nu_{n 0} \vec{\mu}_{0 n} \vec{\mu}_{n 0}}{\nu_{n 0}^{2}-\nu^{2}}
$$

we obtain the $z$ components of $\vec{\mu}_{e_{2} g}$ and $\vec{\mu}_{e_{3} e_{1}}$ as

$$
\begin{array}{r}
\mu_{e_{2} g, z}=\frac{3}{2} R^{-7}\left\{\left(\alpha_{\|}-\alpha_{\perp}\right)\left[X\left(5 Y^{2}-R^{2}\right) \sin 2 \theta \sin \phi+Y\left(5 X^{2}-R^{2}\right) \sin 2 \theta \cos \phi\right]\right. \\
\left.+10 X Y Z\left(\alpha_{\perp} \sin ^{2} \theta+\alpha_{\|} \cos ^{2} \theta\right)\right\} \Theta_{x y}^{e_{2} g}(\mathrm{~A}) \\
\mu_{e_{3} e_{1}, z}=\frac{3}{2} R^{-7}\left\{\left(\alpha_{\|}-\alpha_{\perp}\right)\left[X\left(5 Y^{2}-R^{2}\right) \sin 2 \theta \sin \phi+Y\left(5 X^{2}-R^{2}\right) \sin 2 \theta \cos \phi\right]\right. \\
\left.+10 X Y Z\left(\alpha_{\perp} \sin ^{2} \theta+\alpha_{\|} \cos ^{2} \theta\right)\right\} \Theta_{x y}^{e_{3} e_{1}}(\mathrm{~A}) .
\end{array}
$$

Here $\alpha_{\perp}$ and $\alpha_{\|}$are the perturber's polarizability components perpendicular and parallel to the cylindrical axis of the perturber, respectively. They are evaluated at $\nu=\nu_{e_{2} g}=$ $\left(E_{e_{2}}^{\mathrm{A}}-E_{g}^{\mathrm{A}}\right) / h$ and $\nu=\nu_{e_{3} e_{1}}=\left(E_{e_{3}}^{\mathrm{A}}-E_{e_{1}}^{\mathrm{A}}\right) / h$ for $\mu_{e_{2} g, z}$ and $\mu_{e_{2} e_{1}, z}$, respectively.

Combining the above equations with Eq. (3.9), for OA-SFG induced by the dynamic coupling between the achiral chromophore and the chiral side chain, we get

$$
\begin{aligned}
\chi_{\text {chiral }}^{(2)} \propto F(\omega) R^{-7}\left\{( \alpha _ { \| } - \alpha _ { \perp } ) \left[X\left(5 Y^{2}-R^{2}\right) \sin 2 \theta\right.\right. & \left.\sin \phi+Y\left(5 X^{2}-R^{2}\right) \sin 2 \theta \cos \phi\right] \\
& \left.+10 X Y Z\left(\alpha_{\perp} \sin ^{2} \theta+\alpha_{\|} \cos ^{2} \theta\right)\right\} .
\end{aligned}
$$




\subsection{DYNAMIC COUPLING MODEL WITH AMINO ACIDS AS A MODEL SYSTEM}

Equation (3.16) shows that the induced OA-SFG on the chromophore depends sensitively on the position of the perturber, and the farther away the perturber is from the chromophore, the weaker its ability in inducing chiral response.

Compared with the expression obtained by Höhn and Weigang for the rotatory strength of the carbonyl $n \rightarrow \pi^{*}$ transition [43], Eq. (3.16) has the same dependence on the perturber position and orientation, which is not accidental. Similar to - $\mathrm{COO}^{-}$, the carbonyl group has $C_{2 v}$ symmetry. Its $n \rightarrow \pi^{*}$ transition is magnetic-dipole allowed with the transition moment along $\hat{z}$-axis, and electric-dipole forbidden in all directions. The extrachromophoric perturber induces an electric-dipole transition moment along the $\hat{z}$ direction that determines the strength of $\mathrm{CD}$, just as in the case of OA-SFG from - $\mathrm{COO}^{-}$.

If the nonpolar perturber has an isotropic polarizability with $\alpha_{\|}=\alpha_{\perp} \equiv \alpha$, then Eq. (3.16) can be simplified to the form

$$
\chi_{\text {chiral }}^{(2)} \propto G(\omega) R^{-7} X Y Z \alpha
$$

The relation of $\chi_{\text {chiral }}^{(2)} \propto X Y Z$ shows that the OA-SFG response follows the same octant rule as $\mathrm{CD}[48]$.

To calculate from Eqs. (3.9) and (3.16), we still need to know the values of allowed electric-dipole and quadrupole transition moments of $-\mathrm{COO}^{-}$, which are not quantities generally available. However, we can apply our theory to molecules with the same achiral chromophore but different side chains, such as a series of amino acid molecules, and estimate their relative strength of $\chi_{\text {chiral }}^{(2)}$. In this case, the differences in $\chi_{\text {chiral }}^{(2)}$ of different amino acids come from their different chiral perturbers. The theoretical prediction can then be 


\subsection{EXPERIMENTAL RESULTS FROM AMINO ACIDS WITH} SATURATED SIDE CHAIN

compared with the experimental result.

Before focusing on amino acids, we need to justify the assumption that the perturber has cylindrical symmetry, which apparently is not true for side chains of amino acids. Therefore, in our calculation, instead of treating the extrachromophoric part as a single perturber, we consider each bond in the side chain an independent nonpolar cylindrically symmetric perturber. We then have

$$
\begin{array}{r}
\chi_{\text {chiral }}^{(2)} \propto \Omega \equiv \sum_{\sigma} R_{\sigma}^{-7}\left\{( \alpha _ { \sigma \| } - \alpha _ { \sigma \perp } ) \left[X_{\sigma}\left(5 Y_{\sigma}^{2}-R_{\sigma}^{2}\right) \sin 2 \theta_{\sigma} \sin \phi_{\sigma}\right.\right. \\
\left.\left.+Y_{\sigma}\left(5 X_{\sigma}^{2}-R_{\sigma}^{2}\right) \sin 2 \theta_{\sigma} \cos \phi_{\sigma}\right]+10 X_{\sigma} Y_{\sigma} Z_{\sigma}\left(\alpha_{\sigma \perp} \sin ^{2} \theta_{\sigma}+\alpha_{\sigma \|} \cos ^{2} \theta_{\sigma}\right)\right\}
\end{array}
$$

with $\sigma$ denoting individual bonds. Because $\alpha_{\sigma \|}$ and $\alpha_{\sigma \perp}$, the longitudinal and transverse polarizability components of bond $\sigma$, respectively, were measured with various methods, $\Omega$ can be calculated from Eq. (3.18), if the position and the orientation of each bond are known, and the relative OA-SFG strength from different amino acids can be predicted.

\subsection{Experimental results from amino acids with saturated side chain}

To test the validity of the theory, we measured the OA-SFG of a series of Lamino acid molecules. A picosecond OPG/OPA system pumped by a Nd:YAG laser operated at $20 \mathrm{~Hz}$ was employed to generate tunable UV output $\left(\omega_{1}\right)$ from 250 to $340 \mathrm{~nm}$ (50 $\mu \mathrm{J} /$ pulse), which was directed to overlap with a $1064 \mathrm{~nm}$ beam $\left(\omega_{2}\right)(2 \mathrm{~mJ} / \mathrm{pulse})$ at the exit window of a liquid cell containing amino acids dissolved in $4 \mathrm{M} \mathrm{NaOH}$ solutions. 
The two beams had their incident angles separated by $45^{\circ}$. The SPP polarization combination was used to selectively probe the OA-SFG in the transmission direction. The output sum frequency $\left(\omega_{s}\right)$ wavelength ranged from 207 to $260 \mathrm{~nm}$, which is near the edge of the first absorption band of $-\mathrm{COO}^{-}$. The obtained OA-SFG spectra in terms of the sum frequency wavelength for L-enantiomers of alanine $\left(\mathrm{CH}_{3}-\mathrm{CH}\left(\mathrm{NH}_{2}\right)-\mathrm{COO}^{-}\right)$, valine $\left(\left(\mathrm{CH}_{3}\right)_{2^{-}}\right.$ $\left.\mathrm{CH}-\mathrm{CH}\left(\mathrm{NH}_{2}\right)_{-} \mathrm{COO}^{-}\right)$, leucine $\left(\left(\mathrm{CH}_{3}\right)_{2}-\mathrm{CH}-\mathrm{CH}_{2}-\mathrm{CH}\left(\mathrm{NH}_{2}\right)_{-} \mathrm{COO}^{-}\right)$, isoleucine $\left(\mathrm{CH}_{3}-\mathrm{CH}_{2^{-}}\right.$ $\left.\mathrm{CH}\left(\mathrm{CH}_{3}\right)-\mathrm{CH}\left(\mathrm{NH}_{2}\right)-\mathrm{COO}^{-}\right)$, serine $\left(\mathrm{HO}-\mathrm{CH}_{2}-\mathrm{CH}\left(\mathrm{NH}_{2}\right)-\mathrm{COO}^{-}\right)$, threonine $\left(\mathrm{CH}_{3}-\mathrm{CH}(\mathrm{OH})-\right.$ $\left.\mathrm{CH}\left(\mathrm{NH}_{2}\right)_{-} \mathrm{COO}^{-}\right)$and lysine $\left(\mathrm{H}_{2} \mathrm{~N}-\left(\mathrm{CH}_{2}\right)_{4}-\mathrm{CH}\left(\mathrm{NH}_{2}\right)_{-} \mathrm{COO}^{-}\right)$are shown in Fig. 3.2. The spectral intensity has been normalized by the amino acid concentration. OA-SFG signals from glycine $\left(\mathrm{NH}_{2}-\mathrm{CH}_{2}-\mathrm{COO}^{-}\right.$, an achiral amino acid) and racemic mixtures of chiral amino

acids (not shown here) were below the detection limit, as expected. The $\chi_{\text {chiral }}^{(2)}$ values of all amino acids were found to have the same sign.

Figure 3.2 shows that isoleucine has the strongest OA-SFG response, and the signal intensity of alanine, valine, leucine and isoleucine follows the order of alanine $<$ leucine $<$ valine $<$ isoleucine, uncorrelated with the size of the side chain. Here we adopt the dynamic coupling model to explain the observed sequence more quantitatively, but we first need to know the conformations of these amino acids.

\subsection{Conformation analysis of amino acids}

Although the conformations of amino acids in their crystalline states are known from x-ray crystallography, their conformations in solution, especially for the anionic struc- 


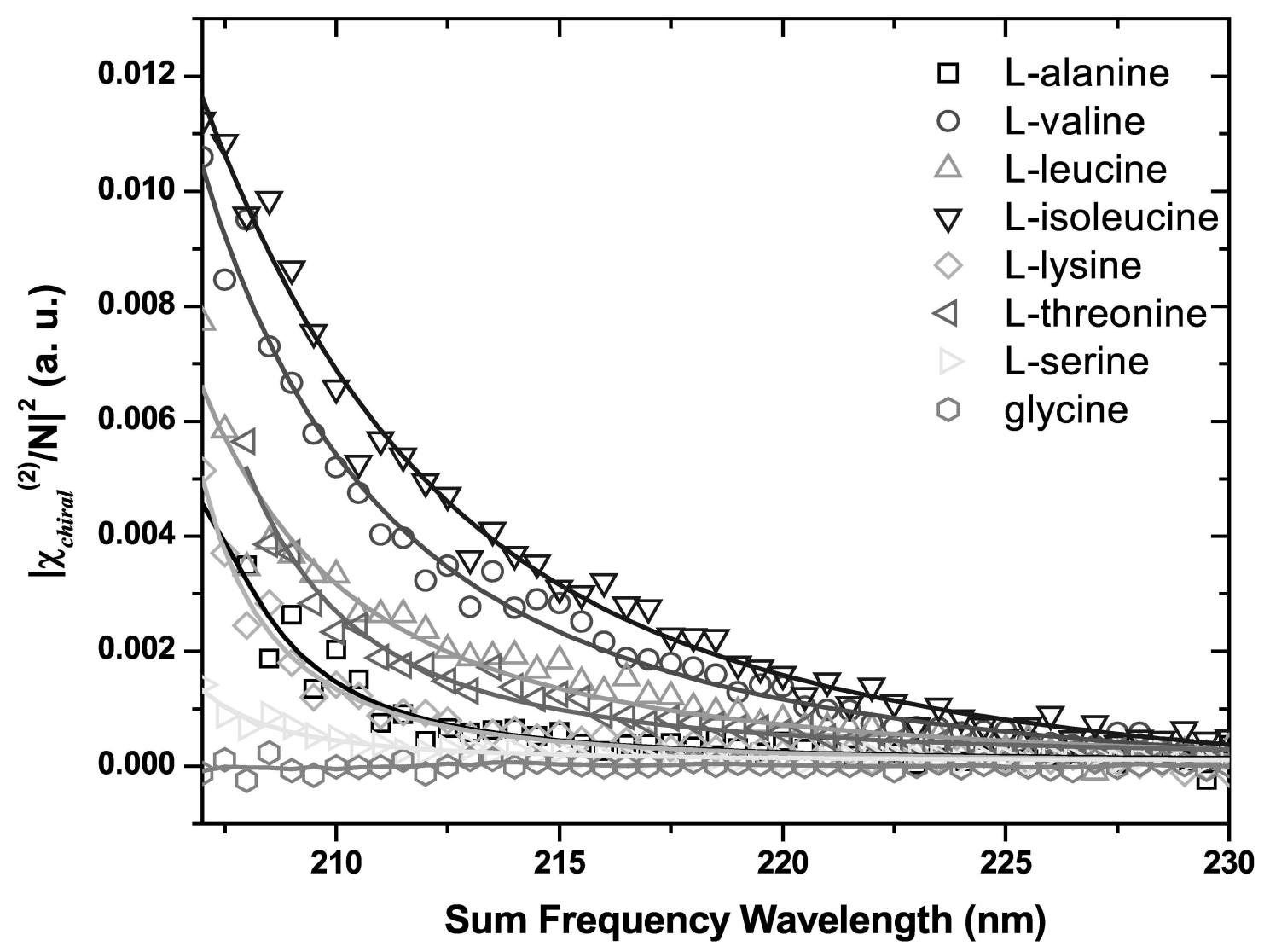

Figure 3.2: OA-SFG spectra, versus the sum-frequency wavelength, of L-enantiomers of alanine, valine, leucine, isoleucine, lysine, threonine and serine, with intensities normalized by the concentration. The OA-SFG signal from the achiral glycine is below our detection limit as expected. Lines are guides to the eye. The polarization combination used is SPP. 
tures we measured here, are not generally known. In principle, molecular conformation can be calculated. For flexible molecules like amino acids, there are many local minima on their potential energy surfaces, corresponding to various possible conformers. All these conformers should be taken into account weighted by their probability distribution in the calculation of molecular properties.

Because amino acids are the building blocks of proteins, studies on the conformers of amino acids have been very active. The gas phase conformations of small amino acids such as glycine and alanine have been calculated by $a b$ initio and density functional methods at different levels $[49,50]$. In aqueous solution, specific interactions between water molecules and the hydrophilic groups of amino acids need to be taken into consideration [44]. Of the most thorough approach to date is to consider a solvation shell made of explicit water molecules, approximate the rest of the solvent molecules as a continuous dielectric environment, find all possible conformers, and calculate their energies. Tajkhorshid et al. [51] and Frimand et al. [52] used density functional methods to obtain the conformational isomers of alanine molecules with 4 and 9 explicit water molecules, respectively. Their results showed that for zwitterionic alanine, the HHO conformer, where one of the oxygen atoms in the $-\mathrm{COO}^{-}$group interacts with two hydrogen atoms in the $-\mathrm{NH}_{3}^{+}$group, has the lowest energy. Comparison between theory and experiment on infrared absorption spectra and vibrational circular dichroism spectra confirmed that the HHO conformer is indeed the dominant species in solution.

For our calculation, instead of attempting to treat the amino acid molecules coupled with the solvent molecules quantum mechanically, we devise an approach that utilizes 

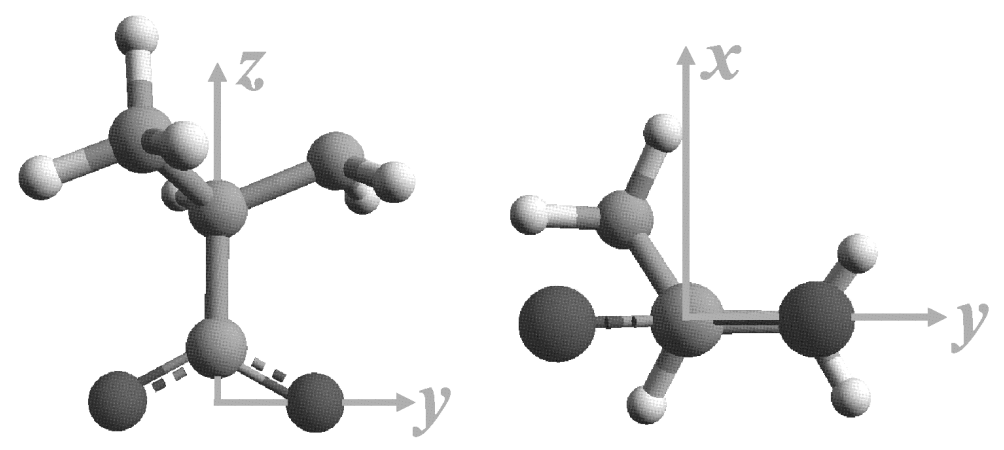

Figure 3.3: Conformation adopted by the hydrophilic $-\mathrm{NH}_{2}$ group and $-\mathrm{COO}^{-}$group on amino acids. Here, L-alanine is presented as an example. Arrows define the molecular coordinate system $x, y, z$.

results from the previous studies. We assume that the hydrophilic groups of the anionic amino acids, similar to their zwitterionic counterparts, adopt the HHO conformation, with an oxygen atom of $-\mathrm{COO}^{-}$group interacting with the two hydrogen atoms in the $-\mathrm{NH}_{2}$ group. We use the same structure parameters - bond lengths, bond angles and dihedral angles - calculated by the B3LYP/6-31G* level theory for the zwitterionic structures in reference [52] to construct our anionic structures, without considering the water molecules explicitly. This is shown in Fig. 3.3 with alanine as an example. Because the hydrophilic groups are those affected most by the specific interactions with water, these parameters should approximate the conformation adopted by these groups in solution reasonably well, and are taken for all the amino acids studied in this paper. Here we assume that the conformation of the hydrophilic groups would not vary much with side chain structures. This assumption is supported by gas phase studies on amino acids, which concluded that changing the side chain of amino acids only has a rather small effect on the conformation 


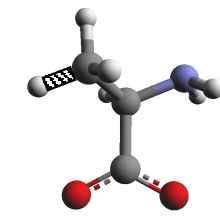

Ala-1

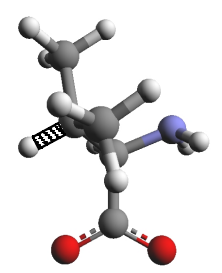

Val-1

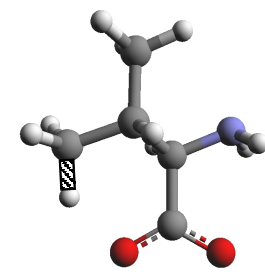

Val-2

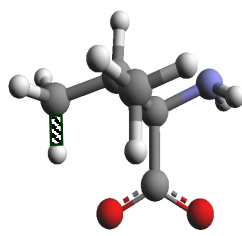

Val-3

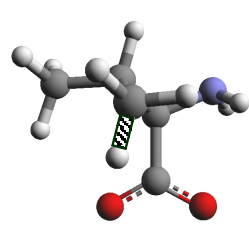

Val-4

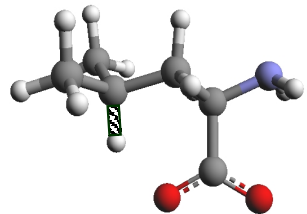

Leu-1

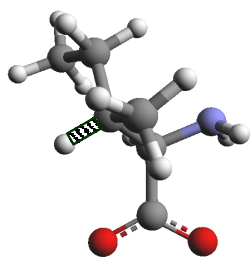

Ile-2

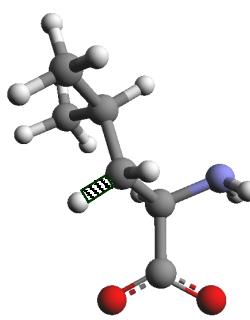

Leu-2

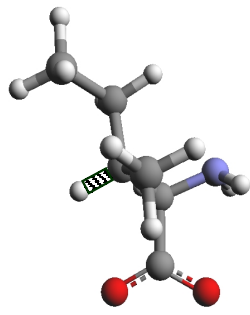

Ile-3

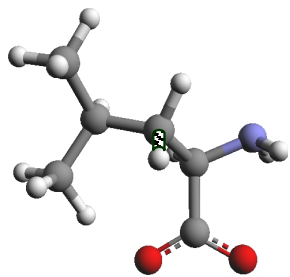

Leu-3

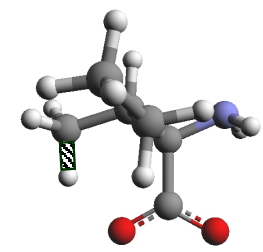

Ile-4

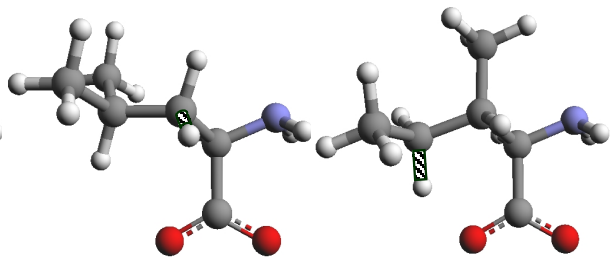

Leu-4

Ile-1

Figure 3.4: Conformational isomers of alanine, valine, leucine and isoleucine. The bonds highlighted with parallel lines are the most effective perturbers in each conformer.

of the hydrophilic groups $[53,54]$. For the hydrophobic side chains, the semiclassical PM3 theory [55] is used to obtain the low-energy conformers without considering the effects of solvation. Considering the weak interactions between hydrophobic side chains and water, the error in neglecting the solvent effects should be small. Indeed, studies on bipeptides $[56,57]$ and glycine amide [58] consistently showed that the lowest energy structures in water and in gas phase are the same. The structures obtained from the above procedure, although to a large extent approximate, should reflect the solvated amino acid structures reasonably well and suffice for our semi-quantitative analysis. 
By assuming the dominant conformation for the hydrophilic groups as the one shown in Fig. 3.3, we only need to identify the low-energy conformational isomers of the hydrophobic side chains that should be included in our calculation. For simplicity, we include only conformers of energies within $1.5 \mathrm{kcal} / \mathrm{mol}\left(2.5 k_{B} T\right.$ at $\left.300 \mathrm{~K}\right)$ above that of the lowest energy conformer identified by the PM3 method. Using the conformation search module in Hyperchem 7 [59], we find that for the hydrophobic side chain, one conformer of alanine, four conformers of valine, four conformers of leucine and six conformers of isoleucine should be included. These conformers are shown in Fig. 3.4 and their coordinates are given in Supporting Information of reference [21].

The above approach is not applicable to serine, threonine, and lysine, due to the presence of hydrophilic groups in their side chains. As will be shown later, without knowing the details of their conformations, we can still explain their OA-SFG intensity qualitatively following the dynamic coupling model.

With their conformations and hence, the position and orientation of each bond in the side chains known, we can calculate from Eq. (3.18) the chiral perturbation strength $\Omega$ for alanine, valine, leucine, and isoleucine. We present our calculation in the following section. 
3.7. APPLICATION OF THE DYNAMIC COUPLING MODEL TO ALANINE, VALINE, LEUCINE, AND ISOLEUCINE

Table 3.1: Polarizabilities of C-C and C-H bonds from literature (in units of $\AA^{3}$ )

\begin{tabular}{ccccc}
\hline \hline & Debign & LeFevre et al. & Allen et al. & Amos et al. \\
\hline$\alpha_{\|}(\mathrm{C}-\mathrm{C})$ & 1.88 & 0.99 & 1.00 & 0.72 \\
$\alpha_{\perp}(\mathrm{C}-\mathrm{C})$ & 0.02 & 0.27 & 0.33 & 0.38 \\
$\alpha_{\|}(\mathrm{C}-\mathrm{H})$ & 0.79 & 0.64 & 0.77 & 0.87 \\
$\alpha_{\perp}(\mathrm{C}-\mathrm{H})$ & 0.58 & 0.64 & 0.70 & 0.49 \\
\hline \hline
\end{tabular}

\subsection{Application of the dynamic coupling model to alanine, valine, leucine, and isoleucine}

As can be seen from the structures in Figs. 3.3 and 3.4, the $\mathrm{C}-\mathrm{N}$ bond lies in the plane of $-\mathrm{COO}^{-}$group, and the two $\mathrm{N}-\mathrm{H}$ bonds of the $-\mathrm{NH}_{2}$ group situate above and below the $-\mathrm{COO}^{-}$plane symmetrically. As a result, their contribution to the perturbation strength $\Omega$ is zero. The only chiral perturbers in these amino acids are the $\mathrm{C}-\mathrm{H}$ and $\mathrm{C}-\mathrm{C}$ bonds in their hydrophobic side chains. If their bond polarizabilities are known, we can use Eq. (3.18) to calculate $\Omega$. Being important parameters for organic molecules, the bond polarizabilities of C-C and C-H bonds have been measured by various groups since the 1930s. The values obtained are more or less consistent for $\mathrm{C}-\mathrm{H}$, but not for $\mathrm{C}-\mathrm{C}$ [60]. Because it is difficult for us to judge which one is correct, we calculate for all the conformers displayed in Fig. 3.4 using four different sets of bond polarizability values from Debign [61], LeFevre et al. [60], Allen et al. [62], and Amos et al. [63] listed in Table 3.1. The results are presented in Table 3.2.

For all four sets of polarizability values, all the calculated $\Omega$ values and, hence, 
Table 3.2: Calculated perturbation strength $\left(\Omega\right.$ in units of $\left.\AA^{-1}\right)$ for 15 conformers of Lalanine, L-valine, L-leucine, and L-isoleucine using different bond polarizability values ${ }^{a}$

\begin{tabular}{cccccc}
\hline \hline structure & $\Delta E(\mathrm{kcal} / \mathrm{mol})^{b}$ & Debign & LeFevre et al. & Allen et al. & Amos et al. \\
\hline Ala-1 $^{c}$ & $\mathbf{0 . 0 0}$ & $\mathbf{- 0 . 0 1 1}$ & $\mathbf{- 0 . 0 1 6}$ & $\mathbf{- 0 . 0 1 8}$ & $\mathbf{- 0 . 0 1 8}$ \\
\hline val-1 $^{c}$ & 0.00 & -0.012 & -0.017 & -0.020 & -0.019 \\
Val-2 $^{c}$ & $\mathbf{0 . 3 7}$ & $\mathbf{- 0 . 0 3 0}$ & $\mathbf{- 0 . 0 3 3}$ & $\mathbf{- 0 . 0 3 8}$ & $\mathbf{- 0 . 0 3 7}$ \\
val-3 $^{\text {val-4 }}$ & 0.81 & -0.021 & -0.028 & -0.034 & -0.038 \\
\hline Leu-1 $^{c}$ & 0.87 & -0.019 & -0.024 & -0.029 & -0.035 \\
Leu-2 $^{\text {Leu-3 }}$ & $\mathbf{0 . 0 0}$ & $\mathbf{- 0 . 0 2 7}$ & $\mathbf{- 0 . 0 3 1}$ & $\mathbf{- 0 . 0 3 6}$ & $\mathbf{- 0 . 0 3 6}$ \\
Leu-4 $_{\text {Ile-1 }}$ & 0.71 & -0.017 & -0.019 & -0.022 & -0.021 \\
Ile-2 & 1.05 & -0.027 & -0.029 & -0.034 & -0.034 \\
Ile-3 & 1.17 & -0.019 & -0.023 & -0.027 & -0.027 \\
Ile-4 & $\mathbf{0 . 0 0}$ & $\mathbf{- 0 . 0 3 3}$ & $\mathbf{- 0 . 0 3 7}$ & $\mathbf{- 0 . 0 4 3}$ & $-\mathbf{0 . 0 4 2}$ \\
Ile-5 & 0.41 & -0.010 & -0.015 & -0.017 & -0.017 \\
Ile-6 & 0.77 & -0.015 & -0.019 & -0.023 & -0.023 \\
\hline \hline
\end{tabular}

${ }^{a}$ The center of gravity for the charge distribution on $-\mathrm{COO}^{-}$is approximated as the midpoint between the two oxygen atoms; the centers of gravity for $\mathrm{C}-\mathrm{C}$ and $\mathrm{C}-\mathrm{H}$ bonds are assumed to be the midpoints of the bonds. ${ }^{b} \Delta E$ is the relative energy of individual conformer compared to the most stable conformer of each amino acid calculated by PM3 theory.

${ }^{c}$ The structures highlighted with bold fonts are those conformers with largest $\Omega$ for each amino acid. 


\subsection{APPLICATION OF THE DYNAMIC COUPLING MODEL TO ALANINE, VALINE, LEUCINE, AND ISOLEUCINE}

$\chi_{\text {chiral }}^{(2)}$ values for L-alanine, L-valine, L-leucine and L-isoleucine have the same sign, in agreement with the experimental observation. Quantitatively, independent of the set of polarizabilities used, the magnitude of $\Omega$ (and hence $\chi_{\text {chiral }}^{(2)}$ ) increases in the order of Ala-1, Leu-1, Val-2 and Ile-1, which are the conformers with the largest $\Omega$ for each amino acids. All being low energy conformers, they are the dominant species in solution. Comparison with Fig. 3.2 shows that the calculated intensity sequence agrees with our experimental result.

As seen from Table 3.2, $\Omega$ varies significantly for different molecular conformations of the same amino acid. An internal rotation along a single $\mathrm{C}-\mathrm{C}$ bond is capable of changing $\Omega$ by three times. This is not surprising, as Eq. (3.18) shows that the chiral perturbation is sensitive to the positions of the perturbers relative to the achiral chromophore. On the other hand, $\Omega$ depends on the linear superposition of the polarizabilities of various bonds and therefore is not very sensitive to the variations in polarizability values. The disagreement in literature on bond polarizability values often originated from the different assumptions used in deducing the values from measurements of the polarizability of whole molecules. We also note that the semiclassical calculation with the PM3 parameter set cannot predict the energies of conformational isomers with high precision. In addition, in aqueous solutions, due to the large dielectric constant of water, the energy differences as well as the inter-conversion energy barriers between different conformers are lowered by the decreased nonbonding interactions between neighboring groups [56, 57, 64]. Considering the uncertainty in the relative energies of the different conformers, we find it satisfying that even if we take just a simple average of $\Omega$ over all conformers of an amino acid, the strength of $\Omega$ still follows the order of alanine $<$ leucine $<$ valine $<$ isoleucine. This strongly suggests 
the validity of our theoretical model.

\subsection{Perturbers most effective in inducing chirality}

Having shown that our dynamic coupling model produces results consistent with our measurements, in the following we wish to explore further and identify the kind of bond perturbers that are most effective in inducing chirality. This knowledge would allow us to predict OA-SFG responses of similar molecules.

From Eq. (3.18), an effective bond perturber should have the following properties:

1. Although the bond must be tilted away from the $-\mathrm{COO}^{-}$plane $(\hat{y}-\hat{z})$, it should also be away from the $\hat{x}-\hat{z}$ plane. In other words, it should be away from both symmetry planes of $-\mathrm{COO}^{-}$to break the achiral symmetry of the chromophore effectively.

2. It should not be too far away from the chromophore, because its perturbation strength from the dynamic coupling between perturber and chromophore decreases rapidly with distance $\left(\propto R^{-4}\right)$.

3. A bond with large polarizability is a more effective perturber, with its perturbation ability depending on its polarizability anisotropy according to the two rules listed below in 4 and 5 .

4. For a bond with isotropic polarizability $\left(\alpha_{\perp}=\alpha_{\|}\right)$, its chiral perturbation strength depends solely on the product of its position coordinates $X Y Z$, leading to an octant rule for OA-SFG, similar to that for CD.

5. For a bond with large polarizability anisotropy (e.g., $\alpha_{\|} \gg \alpha_{\perp}$ ), in addition 
to the dependence on its position, the perturbation strength also depends strongly on its orientation. For $-\mathrm{COO}^{-}$, a bond with large axial polarizability would induce chirality most effectively if the bond orients along the $\hat{z}$-axis. This is because in order to induce a transition dipole moment along $\hat{z}$ for $-\mathrm{COO}^{-}$to have a nonzero chiral response, the perturber should have a polarizability component along the same direction.

The manifestation of the above rules in our system can be appreciated by looking at the perturbation strength of the individual bond. The $\mathrm{C}-\mathrm{C}$ and $\mathrm{C}-\mathrm{H}$ bond perturbation strength for each conformer is listed in Table 2 of Supporting Information in reference [21]. We identify in each conformer the most effective perturber and highlight the bond with parallel lines in Fig. 3.4. Interestingly, all these bonds are $\mathrm{C}-\mathrm{H}$ bonds, with almost all of their $\Omega$ values larger than $0.010 \AA^{-1}$, constituting more than $30 \%$ of the overall perturbation strength for most conformers. These strong perturbers all have properties following the above-mentioned rules; they are tilted sufficiently away from the two mirror planes of the chromophore, while at the same time sufficiently close to the chromophore, with all of them being $\mathrm{C}-\mathrm{H}$ bonds attached to either $\beta$ - or $\gamma$-carbon atoms. This comes as no surprise: the tetrahedral bonding among $\alpha^{-}, \beta$ - and $\gamma$ - carbon atoms moves the $\mathrm{C}-\mathrm{H}$ bonds away from the symmetry planes, whereas keeping them close to the chromophore. Because the polarizability of $\mathrm{C}-\mathrm{H}$ bond is nearly isotropic, its orientation does not affect its perturbation ability much.

The fact that all of these strong perturbers are C-H bonds does not mean that C-C bond is intrinsically weaker perturber. On the contrary, if the $\mathrm{C}-\mathrm{H}$ bond were replaced by $\mathrm{C}-\mathrm{C}$ bond, the perturbation strength would be larger, due to the larger axial polarizability 
of $\mathrm{C}-\mathrm{C}$ bond. In reality, however, the $\mathrm{C}-\mathrm{C}$ bonds at the hydrophobic end of the amino acids are always connected to $\mathrm{C}-\mathrm{H}$ bonds. As a result, steric repulsion prevents them from occupying positions close to the chromophore. The $\mathrm{C}_{\alpha}-\mathrm{C}_{\beta}$ bond is, on the other hand, too close to the symmetry planes of the chromophore and has too large an angle with the $\hat{z}$-axis to contribute significantly.

Now that we understand how C-H and C-C bonds induce chirality in the achiral chromophore, we can go back to Table 3.2 and explain why certain conformers give larger chiral responses than others. As an example, we consider valine, for which Val-2 is the conformer with the largest $\Omega$. As shown in Fig. 3.4, compared with the other conformers of valine, both methyl groups on Val-2 are away from the two symmetry planes, whereas all the other conformers have one of the methyl groups close to the $\hat{x}-\hat{z}$ symmetry plane. For the same reason, the chiral response from Ile- 1 is the strongest among conformers of isoleucine. In fact, Ile-1 has the same structure as Val-2, except that one hydrogen atom on Val-2 is replaced with a methyl group pointing away from the symmetry planes (instead of towards the planes as in Ile-6). Because of the extra methyl group, Ile- 1 has a larger $\Omega$ than Val-2. The lower perturbation strength of leucine conformers can be explained by the absence of methyl substitution at the $\beta$-carbon, resulting in larger distances between the perturbers and the chromophore, and hence less effective chiral perturbation. 


\subsection{Discussion on serine, threonine, and lysine}

$$
\text { Serine }\left(\mathrm{HO}-\mathrm{CH}_{2}-\mathrm{CH}\left(\mathrm{NH}_{2}\right)-\mathrm{COO}^{-}\right) \text {and threonine }\left(\mathrm{CH}_{3}-\mathrm{CH}(\mathrm{OH})-\mathrm{CH}\left(\mathrm{NH}_{2}\right)-\mathrm{COO}^{-}\right)
$$

have hydrophilic polar O-H bonds on their side chains, therefore our dynamic coupling model and procedure for finding favorable conformations are not applicable here. Nevertheless, the model can explain the relative chiral responses of serine and threonine qualitatively. Because serine is transformed to threonine by replacing a hydrogen atom on the $\beta$-carbon with a methyl group, with the assumption of the two having similar conformations in their side chains [54], the difference in their chiral responses should come from the terminal methyl group on threonine. This is similar to replacing one of the hydrogen atoms attached to the $\alpha$-carbon of achiral glycine $\left(\mathrm{NH}_{2}-\mathrm{CH}_{2}-\mathrm{COO}^{-}\right)$with a methyl group to form alanine $\left(\mathrm{CH}_{3}-\mathrm{CH}\left(\mathrm{NH}_{2}\right)-\mathrm{COO}^{-}\right)$. Thus, the difference in chiral responses from threonine and serine is likely to be similar to the chiral response of alanine, as indeed confirmed by the experimental results shown in Fig. 3.2.

Lysine $\left(\mathrm{H}_{2} \mathrm{~N}-\left(\mathrm{CH}_{2}\right)_{4}-\mathrm{CH}\left(\mathrm{NH}_{2}\right)-\mathrm{COO}^{-}\right)$is an amino acid with a $-\mathrm{NH}_{2}$ polar group at the end of the alkyl side chain. Because there is no bulky substitution on its side chain, lysine is very flexible and has many conformational isomers. Without knowing much about its conformation and the role of the polar $-\mathrm{NH}_{2}$ group, based on the number of perturbers

in the vicinity of the chromophore, we estimate $\chi_{\text {chiral }}^{(2)}$ of lysine to be smaller than those of valine, leucine and isoleucine, but comparable to that of alanine. The prediction agrees with the experimental results in Fig. 3.2. 


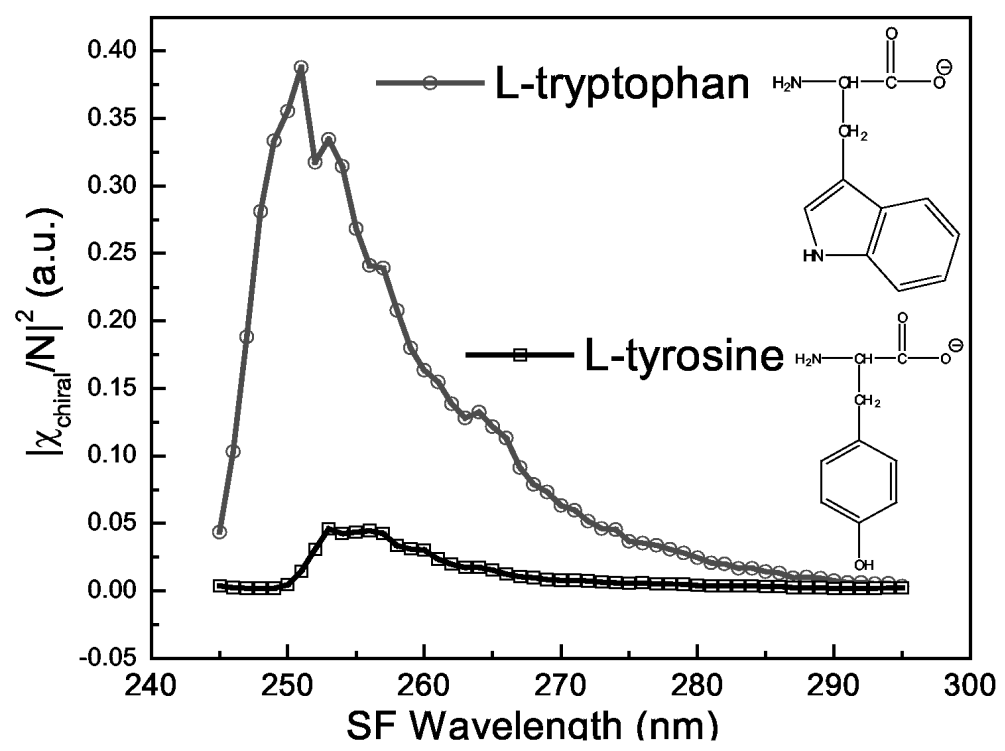

Figure 3.5: OA-SFG spectra of L-tryptophan and L-tyrosine normalized to concentration. Polarization combination is SPP.

\subsection{Experimental results on other amino acids}

We also measured OA-SFG spectra from L-tyrosine and L-tryptophan with the sum frequency in resonance with the electronic transitions of their aromatic side chains. Their concentration-normalized spectra are presented in Fig. 3.5. Similar to the ${ }^{-\mathrm{COO}^{-}}$ group, these intrinsically achiral aromatic groups also show induced OA-SFG from their neighboring chiral perturbers. Tryptophan has a much larger response than tyrosine, which can be partially explained by its more delocalized electrons and the resultant stronger coupling with its chiral perturbers. 


\subsection{Additional considerations}

In our calculation, the bond polarizability values from literature were directly used without the correction for their frequency dependence. For our semi-quantitative evaluation, this approach suffices [65]. However, if we are interested in probing OA-SFG spectra over a wide range, we would have to include the dispersion of the bond polarizability. This is particularly true if the sum frequency approaches resonances of the bonds.

We have also neglected static coupling (see Eq. (3.7)) in our evaluation of amino acids. It gives rise to the one-electron mechanism contributing to the linear optical activity [66], but for systems with saturated side chain, it has been shown to be rather small [67]. Similarly, its contribution to OA-SFG should also be small for the amino acids we have studied.

Although induced chirality in the - $^{-} \mathrm{OO}^{-}$group has been used in our discussion, our model can be employed to predict induced chirality in achiral chromophores by chiral environment in general. Because in this model, all interactions between the achiral chromophore and its chiral perturbers are through space rather than through chemical bonds, it can also be used to predict chirality induced in an achiral molecule by neighboring chiral molecules. Although not yet observed, induced OA-SFG strength from intrinsically achiral molecules could be comparable to that of an achiral chromophore in a chiral molecule.

The CD measurements on the same series of amino acids in the same spectral range $(\sim 210 \mathrm{~nm})$ gave different relative chiral strengths from those of OA-SFG. Instead of alanine $<$ leucine $<$ valine $<$ isoleucine as in $\mathrm{OA}-\mathrm{SFG}, \mathrm{CD}$ has alanine $<$ valine $<$ 
isoleucine $<$ leucine $[65,68,69,70,71]$. In this spectral range, $|g\rangle \rightarrow\left|e_{1}\right\rangle$ and $|g\rangle \rightarrow\left|e_{2}\right\rangle$ are the dominant transitions in CD spectra. As shown in Fig. 3.1(b), $|g\rangle \rightarrow\left|e_{2}\right\rangle$ transition of $-\mathrm{COO}^{-}$is magnetic-dipole allowed in the $\hat{z}$ direction, but electric-dipole forbidden. The presence of nonpolar chiral side chain induces nonzero electric-dipole transition moment between $|g\rangle$ and $\left|e_{2}\right\rangle$ through the same dynamic coupling mechanism, with its $\hat{z}$ component determining the CD magnitude. This would make the CD signal strength depend on the side chain structure the same way as OA-SFG signal strength, if it were not for the additional contribution from transition $|g\rangle \rightarrow\left|e_{1}\right\rangle$. For this transition, in an unperturbed $-\mathrm{COO}^{-}$, the electric- and magnetic-dipole transition moments are allowed but orthogonal. The expression of rotatory strength for this kind of transition has been worked out, in which the dipole-dipole interaction terms between the chromophore and perturbers now contribute, because of the nonzero electric transition dipole moment of the chromophore [67]. This gives rise to a different dependence on the spatial characteristics of the perturbers from that of transition $|g\rangle \rightarrow\left|e_{2}\right\rangle$, thus an overall different relative CD signal strength from that of OA-SFG.

Note that the model here yields a spectrum of $\chi_{\text {chiral }}^{(2)}$ with both magnitude and phase. Because $\chi^{(2)}$ can be obtained by interference methods [72], this model in principle allows us to determine the absolute configuration and conformation for chiral molecules composed of achiral chromophore and chiral side chain. 


\subsection{Summary}

In summary, we present here a general theory for OA-SFG from molecules made of a chiral center and an achiral chromophore in solutions. Adapting an electron correlation model first proposed by Höhn and Weigang for linear optical activity and using a bondadditive model, we are able to quantitatively explain the experimentally observed OA-SFG from amino acids near the electronic resonance of their intrinsically achiral chromophore $\mathrm{COO}^{-}$. The nonlinear chiroptical response is induced by the correlative electron interactions between the achiral chromophore and the chiral side chain, and its magnitude is determined by the spatial arrangement of the chiral perturbers. Some simple rules on the effectiveness of the extrachromophoric structure in inducing chirality in achiral chromophores are proposed. 


\section{Chapter 4}

\section{Towards chiral sum-frequency}

\section{spectroscopy with information on both magnitude and phase}

\subsection{Introduction}

In Chapter 3, we show how sum-frequency generation (SFG) can be used to detect molecular chirality. Because it is electric-dipole allowed, SFG has higher sensitivity than the conventional methods such as circular dichroism (CD) and optical rotatory dispersion (ORD). However, unlike $\mathrm{CD}$ and $\mathrm{ORD}$, which can distinguish enantiomers and produce chiral spectra to provide information on molecular structure and conformation, the usual optically active SFG (OA-SFG) experiment measures only the amplitude but not the phase 


\subsection{PROBLEM OF OPTICALLY ACTIVE SUM-FREQUENCY GENERATION}

of the chiral response, because $\left|\chi_{\text {chiral }}^{(2)}\right|^{2}$ is measured instead of $\chi_{\text {chiral }}^{(2)}$. This is equivalent to obtaining a CD spectrum with only the absolute magnitude but not the sign of the dichroism. As a result, the application of OA-SFG in studying molecular configuration is limited. In this chapter, we describe a general interference scheme that can provide both the magnitude and the phase of the nonlinear optical responses. We apply the scheme to SFG from chiral liquids and present the first nonlinear chiral spectra.

\subsection{Problem of optically active sum-frequency generation}

Under the electric-dipole approximation, the second-order susceptibility $\overleftrightarrow{\chi}^{(2)}$ of an isotropic chiral liquid has six nonzero elements

$$
\chi_{\text {chiral }}^{(2)} \equiv \chi_{\mathrm{XYZ}}^{(2)}=-\chi_{\mathrm{XZY}}^{(2)}=\chi_{\mathrm{YZX}}^{(2)}=-\chi_{\mathrm{YXZ}}^{(2)}=\chi_{\mathrm{ZXY}}^{(2)}=-\chi_{\mathrm{ZYX}}^{(2)}
$$

which have opposite signs for the two enantiomers and zero for a racemic mixture. In conventional OA-SFG, the measured signal has intensity

$$
I_{1}=C\left|\chi_{\text {chiral }, \text { eff }}^{(2)}\right|^{2} \text { with } \chi_{\text {chiral }, \text { eff }}^{(2)}=F_{1} \chi_{\text {chiral }}^{(2)} l_{c}
$$

where $C$ is a constant determined by the experimental geometry, and $\chi_{\text {chiral,eff }}^{(2)}$ the effective chiral susceptibility, which is related to $\chi_{\text {chiral }}^{(2)}$ by the local field correction factor $F_{1}$ and the coherence length $l_{c}$. Electronic or vibrational spectra of the system can be obtained by scanning either one of the input frequencies or the output sum frequency over the transitions, with $\chi_{\text {chiral }}^{(2)}$ in the form of

$$
\chi_{\text {chiral }}^{(2)}=\sum_{n} \frac{A_{n}}{\omega-\omega_{n}+i \Gamma_{n}}+\chi_{\text {chiral,NR }}^{(2)} .
$$




\subsection{PROBLEM OF OPTICALLY ACTIVE SUM-FREQUENCY

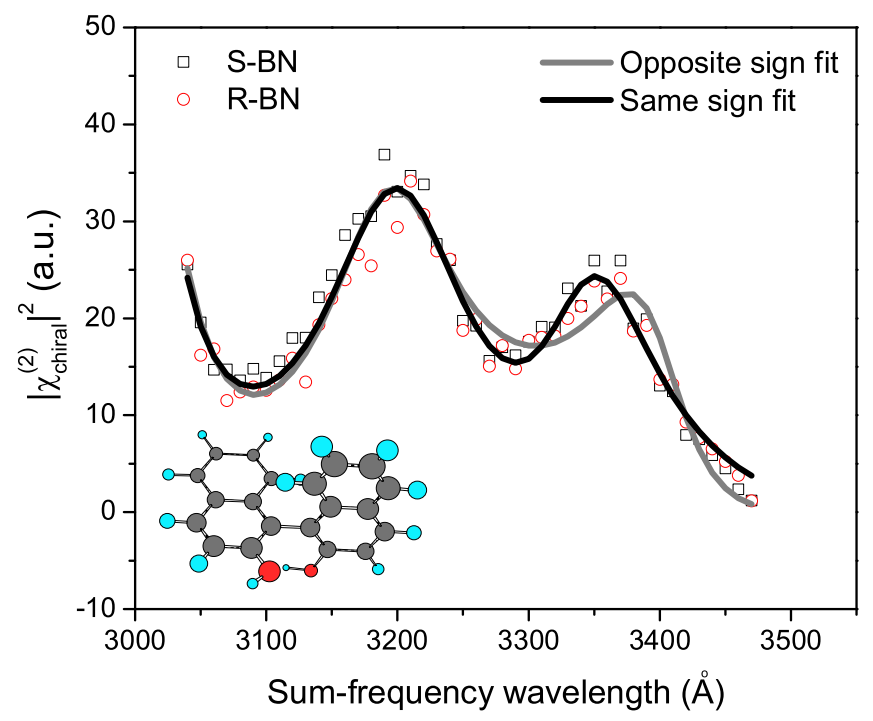

Figure 4.1: $\left|\chi_{\text {chiral }}^{(2)}\right|^{2}$ versus sum frequency wavelength from solutions of S- and R-BN. Black and gray curves are fits to the data points using Eqs. (4.2) and (4.3) with $A_{n}$ 's of the two transitions having the same and opposite signs, respectively. The polarization combination used was SPP. The molecular structure of BN is depicted in the inset.

Here $A_{n}, \omega_{n}$, and $\Gamma_{n}$ denote the amplitude, the resonant frequency, and the damping constant for the $n$th transition, and $\chi_{\text {chiral,NR }}^{(2)}$ describes the nonresonant background. Equation (4.2) shows that the sum-frequency signal is independent of the sign of $\chi_{\text {chiral }}^{(2)}$. Therefore, unlike CD or ORD, OA-SFG can only determine whether the sample is chiral, but not its handedness.

The lack of phase information makes it difficult to interpret the obtained OASFG spectra unequivocally, as demonstrated in the following for a model system. Shown in Fig. 4.1 are the optically active SFG spectra obtained from solutions of S-(-)-1,1'-bi-2- 
naphthol (S-BN) and R-(+)-1,1'-bi-2-naphthol (R-BN) with the sum frequency tuned across the first two excitonic electronic transitions [73]. The spectra from S- and R-BN are identical and can be fit well by Eqs. (4.2) and (4.3) with two different parameter sets - one having the two transition amplitudes of the same sign, the other of opposite signs. Since the sign of the transition amplitude is directly related to the chiral molecular configuration, the lack of phase information here makes it difficult to have an unequivocal interpretation on the configuration of these molecules.

\subsection{Theory of chiral sum-frequency spectroscopy}

Interference schemes have been introduced to generate sum-frequency spectra that can distinguish enantiomers. By interference between chiral and achiral elements of $\overleftrightarrow{\chi}^{(2)}$ $[17,11,74]$, the two enantiomers exhibited different spectra. However, because the achiral elements of $\overleftrightarrow{\chi}^{(2)}$ 's are often also resonantly enhanced in the same frequency region, the deduced spectrum is not the chiral spectrum of the system, but distorted by the unknown magnitude and phase variations of the achiral $\stackrel{\leftrightarrow}{\chi}^{(2)}$ elements over the resonances. As a result, the magnitude and the phase, or equivalently, the real and imaginary parts of $\chi_{\text {chiral }}^{(2)}$ could not be obtained. Chiral spectra similar to those in CD are yet to be obtained.

To obtain the spectrum of $\chi_{\text {chiral }}^{(2)}=\operatorname{Re}\left(\chi_{\text {chiral }}^{(2)}\right)+i \operatorname{Im}\left(\chi_{\text {chiral }}^{(2)}\right)$, one should interfere $\chi_{\text {chiral }}^{(2)}$ with a dispersionless background with a constant phase. This background can be supplied by a quartz crystal in contact with the chiral liquid. The idea and experimental arrangement are similar to those in the scheme developed for phase-sensitive SFVS [72]. 
The sum-frequency signal generated at the quartz-chiral liquid interface region is given by

$$
I=C\left|\chi_{\text {chiral,eff }}^{(2)}-\chi_{\text {Quartz,eff }}^{(2)}\right|^{2}
$$

where $\chi_{\text {Quartz,eff }}^{(2)}$ is real and denotes contribution from quartz, and the negative sign before $\chi_{\mathrm{Quartz}, \mathrm{eff}}^{(2)}$ originates from the phase difference between the sum-frequency signals of the chiral liquid and the quartz (see Appendix A). Because quartz crystal is anisotropic, $\chi_{\text {Quartz,eff }}^{(2)}$ can be adjusted by orienting the quartz crystal properly. By setting $\chi_{\text {Quartz,eff }}^{(2)}=0$, we have

$$
I_{0}=C\left|\chi_{\text {chiral,eff }}^{(2)}\right|^{2}
$$

If the chiral liquid is replaced by an achiral liquid, we have

$$
I_{Q}=C\left|\chi_{\mathrm{Quartz}, \mathrm{eff}}^{(2)}\right|^{2}
$$

We then find

$$
\begin{aligned}
\operatorname{Re}\left(\chi_{\text {chiral }}^{(2)}\right) & =-\frac{I-I_{0}-I_{Q}}{2 I_{Q} F_{1} l_{c}} \chi_{\text {Quartz,eff }}^{(2)} \\
\left|\operatorname{Im}\left(\chi_{\text {chiral }}^{(2)}\right)\right|^{2} & =\left[\left|\chi_{\text {chiral }}^{(2)}\right|^{2}-\left|\operatorname{Re}\left(\chi_{\text {chiral }}^{(2)}\right)\right|^{2}\right]^{1 / 2} .
\end{aligned}
$$

Here, $\operatorname{Re}\left(\chi_{\text {chiral }}^{(2)}\right)$ can be obtained directly from Eq. (4.7), while only the magnitude of $\operatorname{Im}\left(\chi_{\text {chiral }}^{(2)}\right)$ can be deduced from Eq. (4.8). For systems with simple spectral features such as $\mathrm{BN}$, the sign of $\operatorname{Im}\left(\chi_{\text {chiral }}^{(2)}\right)$ can be deduced from the resonant behavior of $\operatorname{Re}\left(\chi_{\text {chiral }}^{(2)}\right)$. For more complicated spectra with multiple overlapping resonances, simultaneous fit on $I_{0}$ and $\operatorname{Re}\left(\chi_{\text {chiral }}^{(2)}\right)$ gives the transition amplitudes, from which the sign of $\operatorname{Im}\left(\chi_{\text {chiral }}^{(2)}\right)$ could be determined unambiguously. 


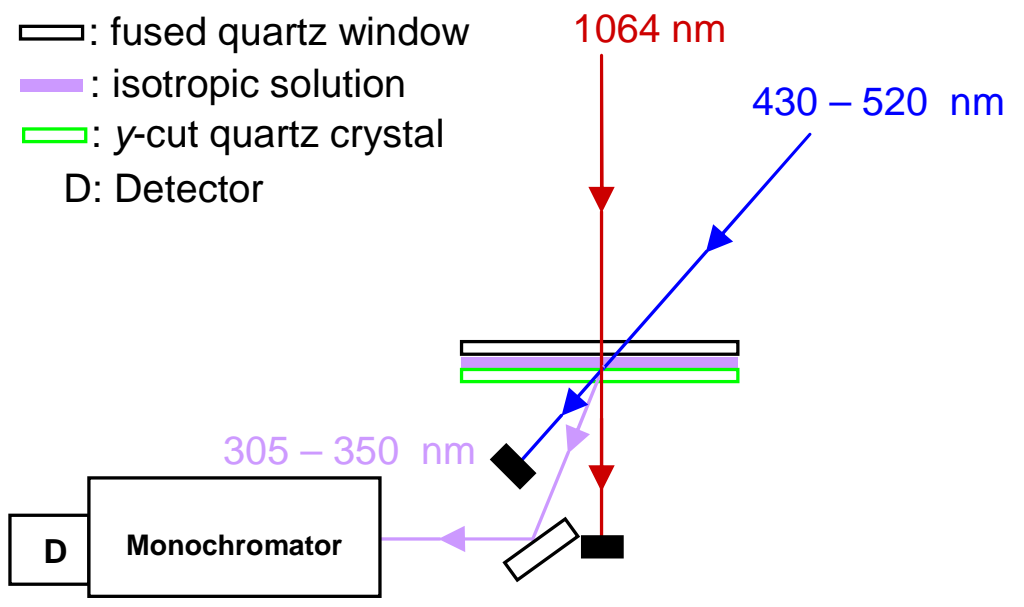

Figure 4.2: Beam geometry of the experimental setup. Input beams were overlapped at chiral solution-quartz interface, and sum-frequency signal was detected in the transmission direction. Polarization combination was SPP.

\subsection{Experimental arrangement}

As a demonstration, we measured the chiral electronic sum-frequency spectra from S- and R-BN solutions. The experimental arrangement is schematically shown in Fig. 4.2. The tunable visible beam was generated by a home-made picosecond OPG/OPA pumped by a Nd:YAG laser (PY61C-20, Continuum Inc.) operated at $20 \mathrm{~Hz}$. The $1064 \mathrm{~nm}$ beam was the direct output of the Nd:YAG laser. The typical pulse width was around 20 picoseconds, and the energies per pulse were $2.0 \mathrm{~mJ}$ for $1064 \mathrm{~nm}$ and $300 \mu \mathrm{J}$ for visible beams. The sample solution was sandwiched between a fused quartz window and a y-cut crystalline quartz plate, which was used as the source of the dispersionless background. The two input beams were overlapped spatially and temporally at the solution-y-cut-quartz interface. The incident angles are $0^{\circ}$ and $45^{\circ}$ for $1064 \mathrm{~nm}$ and visible beams, respectively. Spectra of $I$, 
$I_{0}$, and $I_{Q}$ versus sum-frequency wavelength were recorded with a detector in the phasematched transmission direction after spatial and spectral filtering. The input beams were P-polarized, while S-polarized sum frequency was detected.

S-BN, R-BN and tetrahydrofuran were purchased from Sigma-Aldrich. S-BN and R-BN solutions were prepared by dissolving BN samples in tetrahydrofuran. Racemic mixture was prepared by dissolving equal amount of S-BN and R-BN, and was used as the achiral liquid in the measurement of $I_{Q}$. The concentration used was $0.3 \mathrm{M}$.

\subsection{Results and discussion}

From the measured spectra, $\operatorname{Re}\left(\chi_{\text {chiral }}^{(2)}\right)$ and $\operatorname{Im}\left(\chi_{\text {chiral }}^{(2)}\right)$ for S- and R-BN were deduced and presented in Fig. 4.3. S-BN and R-BN have opposite signs in both their real and imaginary components of $\chi_{\text {chiral }}^{(2)}$, as expected. These are the first nonlinear optical chiral spectra ever reported. The spectra were fitted by Eq. (4.3) with two resonant peaks at $320 \mathrm{~nm}$ and $340 \mathrm{~nm}$, arising from transitions between the ground state and the first pair of exciton-split excited states of BN [23]. Unlike the conventional OA-SFG spectra in Fig. 4.1, the chiral sum-frequency spectra in Fig. 4.3 show unequivocally that the amplitudes of the exciton-split peaks have opposite signs.

It is known in CD that pairs of exciton-split transitions in chiral molecules consisting of two identical chromophores generally give rise to bisignate CD spectra with peaks showing consecutive positive and negative Cotton effects [3]. The locations of the peak and the trough have been used to derive the absolute configuration and conformation of many 


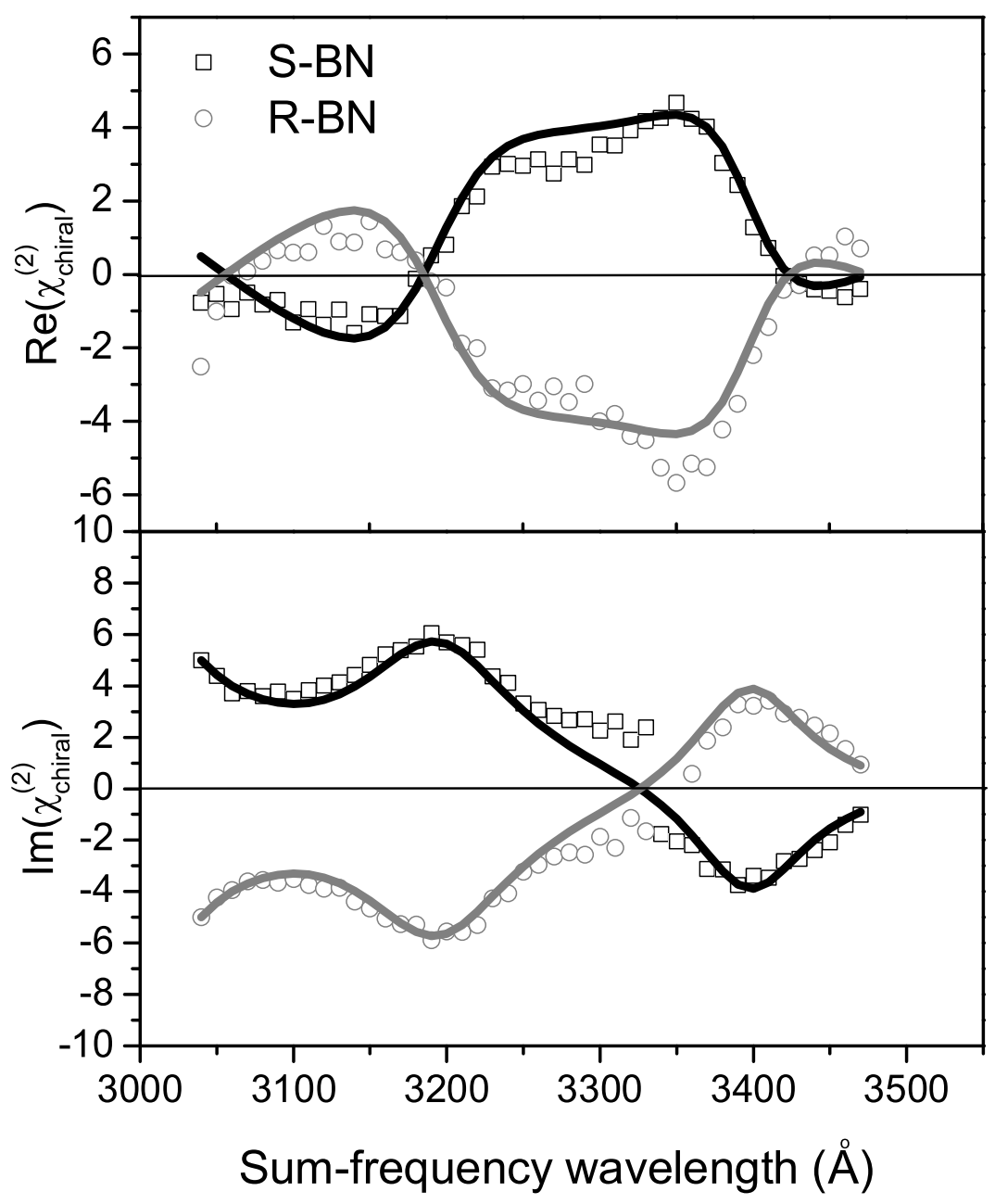

Figure 4.3: Spectra of real and imaginary components of $\chi_{\text {chiral }}^{(2)}$ over the first two excitonsplit transitions of ( $\square$ ) S- and (O) R-BN. The curves are fits to the data with Eq. (4.3) with the same set of parameters that yield the gray curve in Fig. 4.1. 
organic compounds. Similarly, in SFG, a theory based on the coupled-oscillator model predicts that pairs of exciton-split peaks should also exhibit the bisignate feature in $\chi_{\text {chiral }}^{(2)}$, although the structural parameters that produce the linear and nonlinear chiral responses are different [42]. As shown in Fig. 4.3, this bisignate behavior is indeed observed in $\chi_{\text {chiral }}^{(2)}$. In our experiment, we assume the $\chi_{\text {Quartz,eff }}^{(2)}$ in Eq. (4.7) to be positive. At first look, this assumption seems arbitrary. However, since it is difficult to determine the sign of $\chi_{\text {Quartz,eff }}^{(2)}($ even for the same piece of quartz, flipping the quartz upside down would generate a $\chi_{\text {Quartz,eff }}^{(2)}$ with opposite sign), BN molecules with the sign of $\chi_{\text {Quartz,eff }}^{(2)}$ so defined may serve as references to calibrate the sign of $\chi^{(2)}$ from other dispersionless background. Since the conformations of S- and R-BN are known, for other chiral molecules with their chiral sum-frequency spectra obtained similarly, we can now determine their configuration or conformation.

The chiral sum-frequency spectroscopic method described here is generally applicable to all chiral media, but is more convenient in the spectral region where the quartz crystal is transparent so that the dispersion of $\chi_{\text {Quartz,eff }}^{(2)}$ is weak and smooth. Note that analogous to the heterodyne technique, our sensitivity can also be significantly enhanced through interference of $\chi_{\text {chiral }}^{(2)}$ with an appreciably larger $\chi_{\text {Quartz,eff }}^{(2)}$, as can be seen by comparing $I-I_{0}-I_{Q}$ with $I_{0}$. 


\subsection{Summary}

We develop a phase-sensitive SFG method that yields both real and imaginary components of $\chi_{\text {chiral }}^{(2)}$, equivalent in linear optics to obtaining results from both ORD and CD. Considering also its intrinsically high sensitivity, we expect that this chiral sumfrequency spectroscopy could become a powerful analytical tool for studying molecular chirality. 


\section{Chapter 5}

\section{Three-dimensional chiral imaging}

\section{by sum-frequency generation}

\subsection{Introduction}

The majority of biologically important chemical species are chiral. Their functionality is often determined by their spatial arrangements, which are almost always homochiral for each species: Proteins are made of right-handed $\alpha$-helix and left-handed $\beta$-sheets; DNA exists as right-handed double helix in stable physiological conditions [7]. The detection and characterization of chirality on both molecular and conformational levels provide important structural and functional information of biomolecules [75]. Visualization of the three-dimensional (3D) arrangements and placements of these molecules in cells would allow one to directly relate their conformational architectures to biological processes. To achieve this goal, an imaging technique capable of probing chirality is needed. 
Many optical methods have been developed to characterize chirality [3]. Familiar examples include those based on optical rotatory dispersion, circular dichroism, and Raman optical activity. These techniques make use of the differential responses (e.g., refractive indices, absorption coefficients, Raman scattering cross sections) that left- and right-circularly polarized light may experience in chiral media, and have made enormous contributions to chemical analysis and to our understanding of molecular conformations. Microscopy based on circular dichroism contrast was developed, and has been used to obtain images of cells and inorganic chiral crystals $[76,77,78]$. The conventional chirality probes, however, involve higher-order interactions between molecules and light such as magnetic-dipole and electricquadrupole interactions. As a result, the signal tends to be orders-of-magnitude weaker than the chirality-insensitive, electric-dipole allowed background, making them inefficient contrast mechanisms in microscopy.

Recently, optically active sum-frequency generation (OA-SFG) processes have been demonstrated as novel probes of chirality. It was used to obtain optically active vibrational and electronic spectra from chiral liquids [17, 18, 19, 20, 21], thin films [22], and even monolayers[19, 23]. Being electric-dipole allowed, it has intrinsically good contrast to image chiral objects against an achiral background, such as water in a cell, and therefore has the potential of affording stain-free cellular imaging. In this chapter, we report the first 3D OA-SFG microscopy. We used 1,1'-bi-2-naphthol (BN) in tetrahydrofuran as a model system to demonstrate that optically active images with a spatial resolution close to the diffraction limit can be obtained.

The theory of OA-SFG has been described in Chapter 2. Briefly, under the electric- 
dipole approximation, SFG is allowed in a medium without inversion symmetry, as in the case for a chiral material. The sum-frequency signal at $\omega_{s}$ generated by overlapping incoming beams of frequencies $\omega_{1}$ and $\omega_{2}$ in a chiral liquid is proportional to $\left|\chi_{\text {chiral }}^{(2)}\right|^{2}$, where $\chi_{\text {chiral }}^{(2)}$ is the nonlinear susceptibility of the chiral liquid and the beam polarizations are taken to be SPP, PSP, or PPS. $\chi_{\text {chiral }}^{(2)}$ is of opposite signs for enantiomers, and is zero for racemic mixture and achiral media. With resonant enhancement, OA-SFG can provide chemical selectivity.

\subsection{Materials and sample preparation}

R-BN, S-BN and tetrahydrofuran (THF) were purchased from Aldrich and used as received. The $0.45 \mathrm{M} \mathrm{BN}$ solution was prepared by dissolving $7.1 \mathrm{mg}$ solid $\mathrm{BN}$ in $54 \mu \mathrm{L}$ THF, followed by vigorous shaking to ensure a complete dissolution. Racemic BN solution was prepared by mixing equal amount of $0.45 \mathrm{M}$ R- and S-BN solutions. For intensity measurements, pure BN solution was sandwiched between a glass slide and a clean fused quartz slide. For images, silica beads were immobilized on the fused quartz slide to introduce negative contrast. One milliliter solution of $2.4 \mu \mathrm{m}$ diameter silica beads, purchased from Bangs Laboratory, was cleaned using the centrifugal apparatus (Sorvall RT7) with a filter (YM-100 centricon from Millipore) spinning at 3000 RPM for 5 minutes. The clean beads were collected by reversing the centricon and again spinning at 3000 RPM for 2 min. By adding $100 \mu \mathrm{L}$ filtered water (NANOPure Diamond UV/UF, PH 5.5-6), a solution of clean beads was formed. Beads from $5 \mu \mathrm{L}$ of the solution were then spin-coated on a clean quartz 


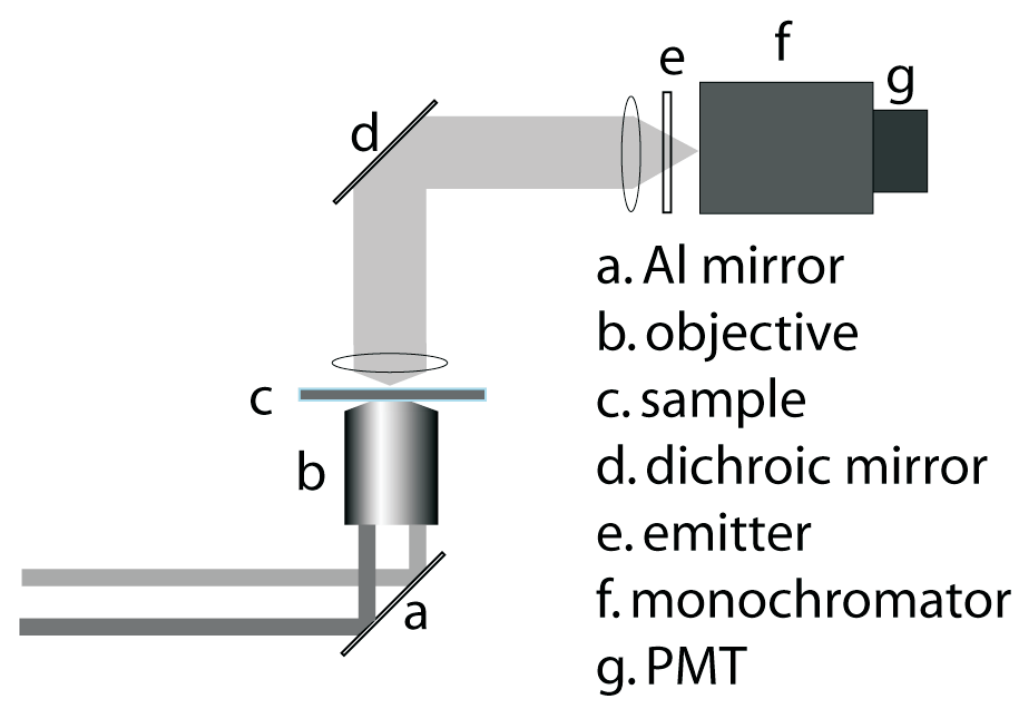

Figure 5.1: Schematic of the microscope.

slide (Technical Glass) at 3500 RPM for 10 seconds. This procedure typically generated a surface density of about $2-4$ beads per $10 \times 10 \mu \mathrm{m}^{2}$. A typical sample cell had $3 \mu \mathrm{L}$ of $\mathrm{BN}$ solution sandwiched between a clean glass slide and a clean fused quartz slide, and Valap (1:1:1 Vaseline/paraffin/ lanolin) was used to seal the cell.

\subsection{Microscope setup}

The optical microscope was similar to that described by Zhang et al. [79]. It is schematically shown in Fig. 5.1. Briefly, $\sim 70$-fs excitation pulses at $830 \mathrm{~nm}$ were generated by a broad-band Ti:sapphire oscillator (Spectral Physics Tsunami) pumped by a diode laser (Spectral Physics Millennia XsJ). The output was split into 2 beams, one of which was frequency doubled by $\alpha$-BBO crystal to generate pulses at $415 \mathrm{~nm}$. The beams were collimated and directed into a microscope with a center-to-center parallel displacement of $3.2 \mathrm{~mm}$. A home-made microscope was used for the two-dimensional(2D) imaging scan, whereas a 
modified commercial microscope (Olympus IX71) was used for 3D sectioning. For the former, the two beams were reflected by an $\mathrm{Al}$ mirror (New Focus, 5108) and focused on the sample through a microscope objective (Olympus PlanApo, 60X, 1.4 NA, oil immersion). The SFG output from the sample was collected in the transmission direction by a CaF2 UV objective, sent through a short-pass filter (Chroma, UG11) and a monochromator (Jobin Yvon-SPEX, H10 D UV), and detected by a UV-sensitive photon-counting PMT (Hamamatsu, H6240). A piezoelectric-controlled stage (Physik Instrumente, P731) was used to raster-scan images. For 3D sectioning, a microscope objective (Optics for Research, LMU40X) was used to collect the SFG output. For spectral analysis of the sum-frequency output, a spectrograph (Acton Research, SP2155) was used. The sectioning was performed using a 3D piezo translation stage (Physik Instrumente, P573.3CL).

\subsection{Results and discussion}

OA-SFG from enantiomeric BN solutions is confirmed spectroscopically. With the input beam wavelengths centered at $415 \mathrm{~nm}\left(\omega_{1}\right)$ and $830 \mathrm{~nm}\left(\omega_{2}\right)$, the sum-frequency output $\left(\omega_{s}\right)$ should be centered at $277 \mathrm{~nm}$. Since $\omega_{s}$ is in resonance with an electronic transition of $\mathrm{BN}$ [73], the OA-SFG signal was resonantly enhanced and therefore easily detectable with the SPP polarization combination. The frequency scan in Fig. 5.2(a) shows that the detected SFG signal indeed has a spectral distribution centered at $277 \mathrm{~nm}$, as expected from the spectral distributions of the input waves displayed in Figs. 5.2(b) and 5.2(c). Figure 5.3 shows that the signal has a linear dependence on input intensities $I_{1}$ and $I_{2}$, as expected. 


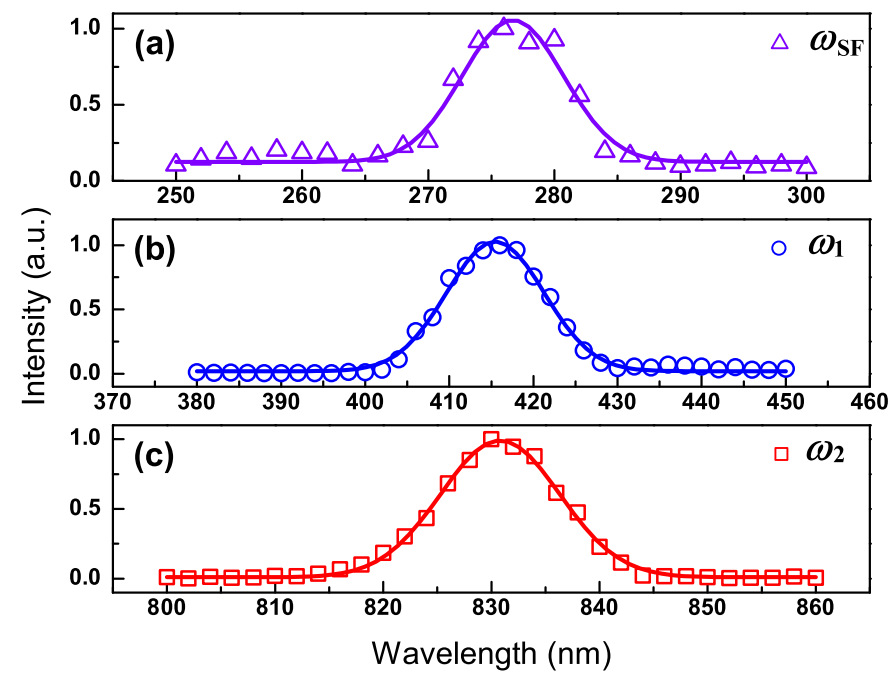

Figure 5.2: Spectra of (a) output sum frequency signal $\left(\omega_{s}\right)$, (b) input wave $\omega_{1}$, and (c) input wave $\omega_{2}$ from R-BN solutions. The polarization combination is SPP. The inset in (a) is the structure of a $\mathrm{R}-\mathrm{BN}$ molecule.
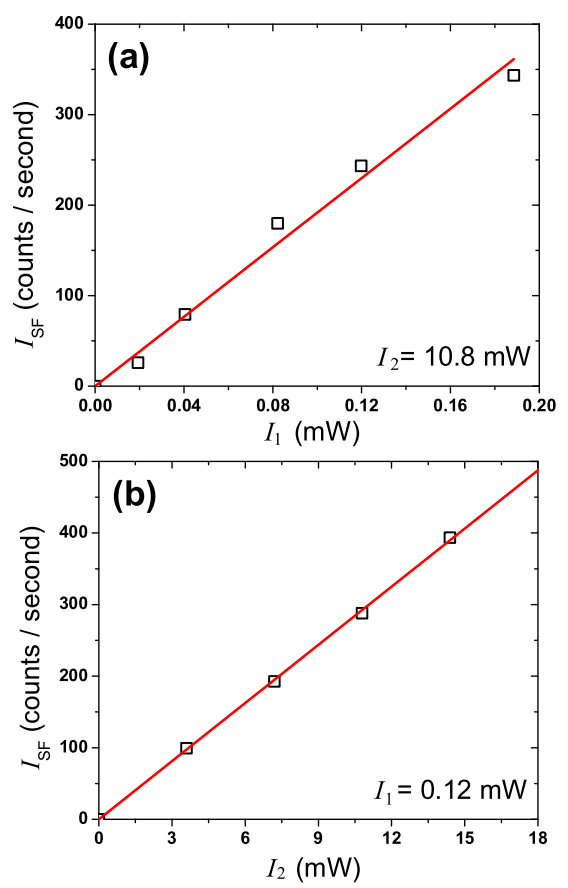

Figure 5.3: OA-SFG signal dependence on (a) $I_{1}$ with $I_{2}$ constant and (b) $I_{2}$ with $I_{1}$ constant. The polarization combination is SPP. 
Under our experimental condition, when the entire effective interaction region is in the chiral solution, sum-frequency signal vanishes. The maximal sum-frequency signal occurs when only half of the interaction region is in the solution. This seemingly surprising behavior is generally true for wave mixing in tight focusing geometry and has been well documented $[80,81,82]$. Similar to the case of third harmonic generation, for example [27, 83], the SFG output can be written as

$$
P_{\mathrm{SF}} \propto|J|^{2}
$$

with

$$
J=\int_{-\infty}^{\infty} \frac{\chi^{(2)}(z) e^{i \Delta k b z}}{1+2 i z} d z
$$

where $z$ is the axial position with $z=0$ being the focal plane, $b$ is the depth of the focal region, $\Delta \vec{k}=\vec{k}_{1}+\vec{k}_{2}-\vec{k}_{s}$ is the wavevector mismatch, and the denominator in the integrand of $J$ is a characteristic of Gaussian beam propagation through the focal region. ${ }^{1}$ For an isotropic medium, it can be shown that $J$ vanishes when $\Delta k$ is smaller than $0[27,83]$, which is the case for BN. Thus when the focal region is in chiral BN solution, SFG vanishes. When only half of the focal region is in the chiral BN solution, $J$ is maximized [83], and thus SFG signal is maximized.

To demonstrate the chiral sensitivity of our SFG imaging, we prepared a sample in which an enantiomeric solution and a racemic solution of $\mathrm{BN}$ are separated by a $55-\mu \mathrm{m}$ wide spacer made of a melted glass fiber. The microscopic image in Fig. 5.4(a) is obtained

\footnotetext{
${ }^{1}$ The formulation here is for collinear sum-frequency generation. For our noncollinear scheme, there would be an extra coefficient in the integration describing the overlapping of the two input beams. The conclusion, however, is the same, as shown in Appendix A.
} 

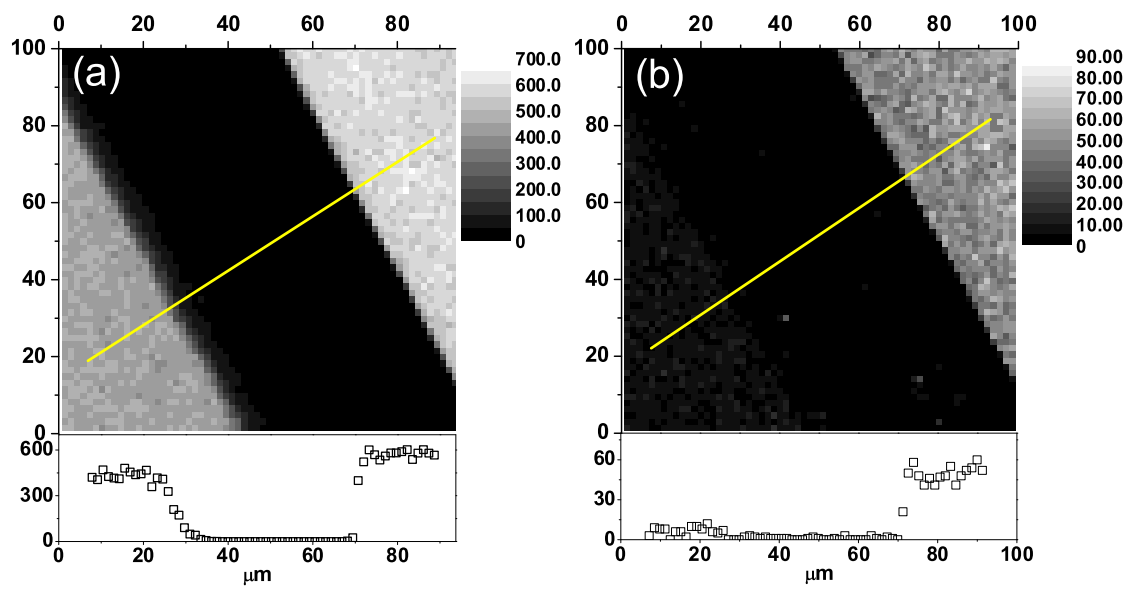

Figure 5.4: (a) Fluorescence image of a racemic BN solution and a R-BN solution separated by a $55-\mu \mathrm{m}$ glass spacer. (b) OA-SFG image of the same sample measured with SPP polarization combination. Bottom panels are signal profiles along the lines in the images. The $100 \mu \mathrm{m} \times 100 \mu \mathrm{m}$ images were taken with collection times of (a) $4 \mathrm{~ms} /$ pixel and (b) 78 $\mathrm{ms} /$ pixel and power levels of $0.24 \mathrm{~mW}\left(\omega_{1}\right)$ and $18 \mathrm{~mW}\left(\omega_{2}\right)$.

by detecting fluorescence from $\mathrm{BN}$ at $350 \mathrm{~nm}$. Since the fluorescence is not sensitive to chirality, both enantiomeric and racemic sections appear bright, as shown in the figure. When the same sample is imaged by OA-SFG, only the enantiomeric section generates strong signal, as shown in Fig. 5.4(b). The achiral racemic solution does generate minimal yet detectable output at $\omega_{s}$, which may be due to the resonantly enhanced parametric light scattering from BN [84].

To characterize the spatial resolution of our microscope, we imaged enantiomeric BN solutions sandwiched between slides decorated with $2.4 \mu \mathrm{m}$ diameter silica beads. A reduction in signal is observed when the achiral beads are in the focal area. Two typical images, one with a single bead and the other with three beads, are displayed in Figs. 5.5(a) 

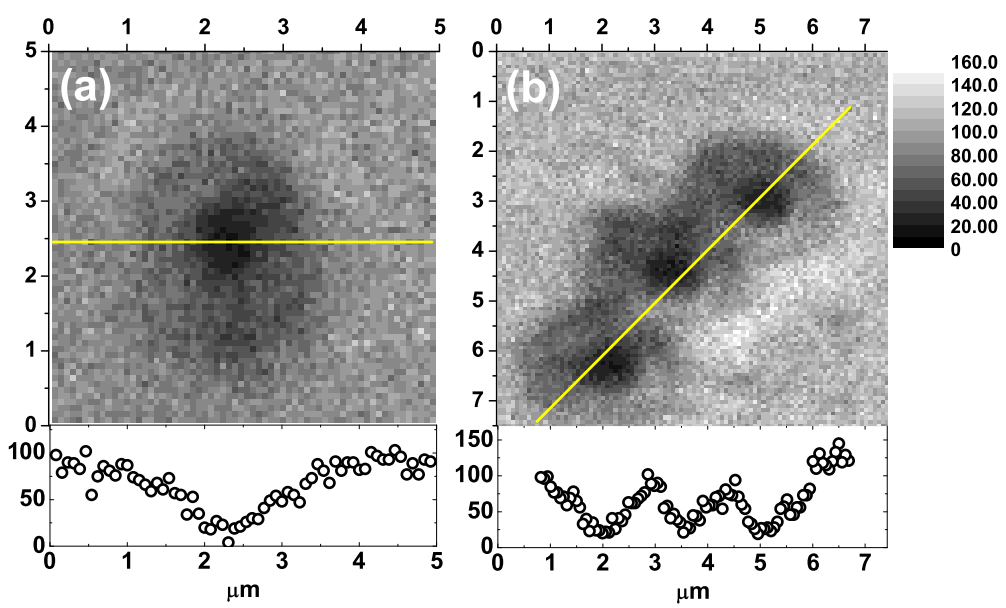

Figure 5.5: SFG images of (a) one and (b) three 2.4- $\mu \mathrm{m}$ diameter silica beads in R-BN solutions. Bottom panels show signal variations along the lines in the images. The polarization combination used was SPP and the collection time per image was $78 \mathrm{~ms} /$ pixel at power levels $0.18 \mathrm{~mW}\left(\omega_{1}\right)$ and $14 \mathrm{~mW}\left(\omega_{2}\right)$.

and 5.5(b), respectively. From the intensity profiles and the focal profile of the objective lens [85], we estimate that the lateral resolution of our microscopy is below $400 \mathrm{~nm}$. This sub-micron resolution makes it easy to resolve the three beads in Fig. 5.5(b).

As a nonlinear optical process where signal is generated only in regions with high enough intensity, OA-SFG microscopy allows 3D sectional imaging. Figure 5.6 presents three sectional images of a sample at different vertical positions, together with cartoons illustrating the interaction regions of light with the sample. As explained previously, when half of the interaction region is in solution, as in Fig. 5.6(b), the chiral signal is the strongest. The signal greatly reduces if a bead appear in the interaction region, giving rise to an image of the beads similar to those seen in Fig. 5.5. Lowering the sample decreases the interaction region of light with solution and accordingly, the signal drops sharply. As illustrated in 

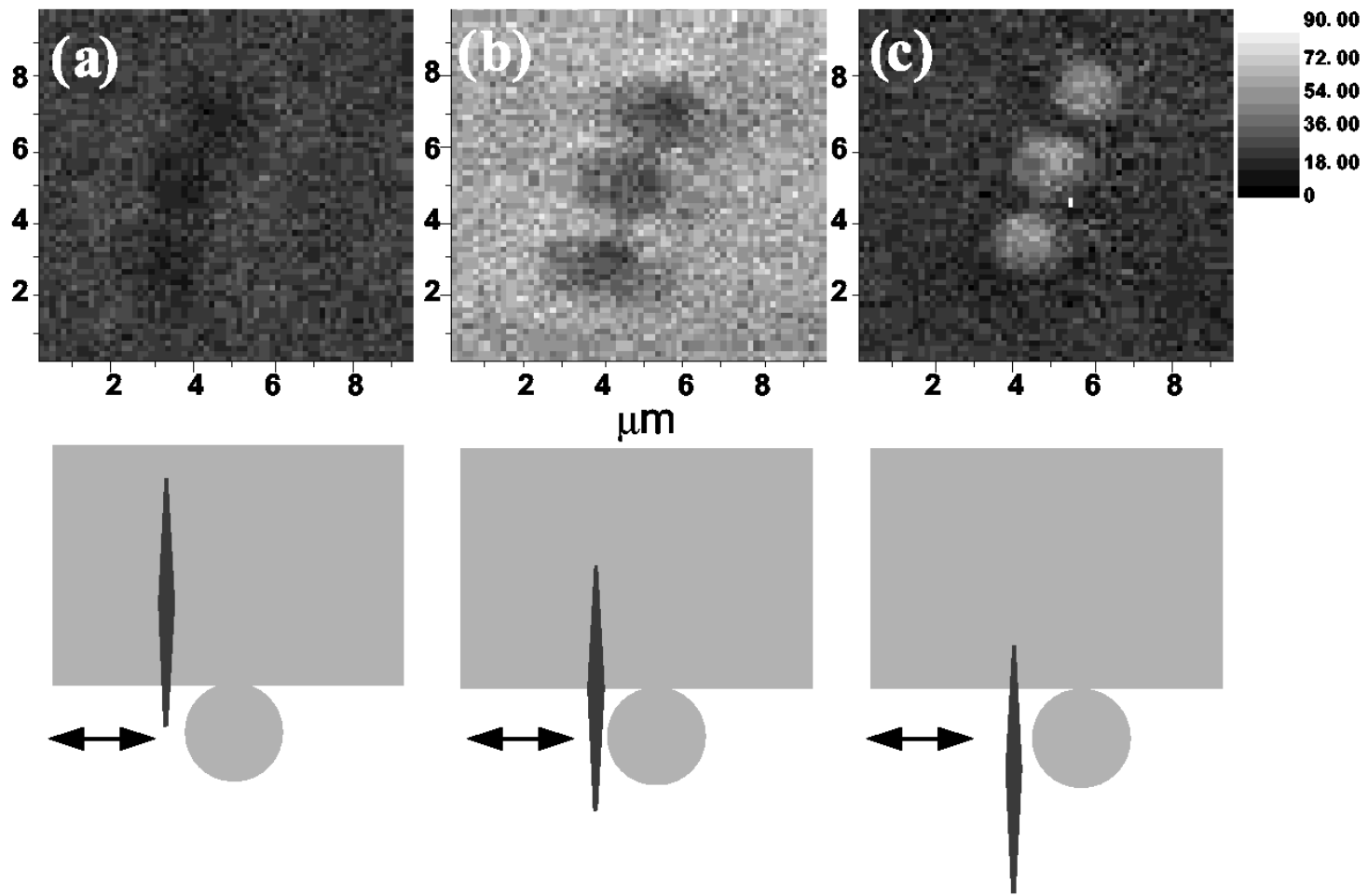

Figure 5.6: SFG images of three 2.4- $\mu$ m diameter silica beads in R-BN solutions with the sample at different vertical positions. Image (b) was obtained with the SFG signal from solution maximized; Images (a) and (c) were obtained with the sample vertically displaced by $+2 \mu \mathrm{m}$ and $-2 \mu \mathrm{m}$ relative to (b), respectively. At bottom are cartoons showing the relative positions of the interaction region (black) and the sample (grey for fused silica slides and beads, white for R-BN solutions). Black arrows denote scanning direction. The polarization combination is SPP and the collection time is $78 \mathrm{~ms} /$ pixel at power $0.24 \mathrm{~mW}$ $\left(\omega_{1}\right)$ and $18 \mathrm{~mW}\left(\omega_{2}\right)$. 
Fig. 5.6(a), although at a much lower contrast the beads could still be resolved. Raising the sample to have the focal region well immersed in solution also sharply decreases the chiral signal from the solution, as shown in Fig. 5.6(c). This is because the sum-frequency signal generated in the focal region experiences destructive interference. Scanning the focal region over the beads now enhances the sum-frequency signal because with part of the focal region in the bead, the total cancelation of sum-frequency signal due to destructive interference is avoided. This allows the beads to stand out as positively contrasted objects in the image.

Second harmonic generation (SHG), a special case of SFG in which two input photons are from the same beam, has been applied to image interfacial systems [86] or non-centrosymmetric ordered structures (e.g., cytoskeleton) [87, 88]. Even though SHG is not allowed in bulk chiral liquids because of the degenerate nature of the input beams, ${ }^{2}$ it can be used to probe chirality from an interfacial layer. This effect has been demonstrated by Petralli-Mallow et al. with a monolayer of BN molecules [8]. More recently, a chiral SHG (C-SHG) microscope has been developed by Kriech et al., with which chiral images of BN monolayers have been obtained [89]. This technique, however, cannot be used to probe bulk chirality, because C-SHG is forbidden in isotropic chiral media. Our microscopic setup actually permits simultaneous measurements of chiral SHG and SFG from a sample, and therefore would allow both surface and bulk chiral imaging of the same chiral system. This is important because chiral molecular arrangements and compositions at the surface and in the bulk are expected to be very different.

Other higher-order, nonlinear optical processes that have been utilized in mi-

\footnotetext{
${ }^{2}$ Note in Eq. (2.15), if $\omega_{1}=\omega_{2}$ as in the case of SHG, $\chi_{\text {chiral }}^{(2)}=0$.
} 
croscopy but are not sensitive to chirality include third harmonic generation (THG) [81] and coherent anti-Stokes Raman scattering (CARS) [90, 91]. These approaches also produce images with sub-micron resolution, are capable of 3D sectioning, and can provide spectroscopic information. In addition to these salient features in microscopy, our OA-SFG microscopy probes only the optically active response of the sample and therefore has the unique advantage that background signal from achiral species such as water is suppressed. Just as CD is a much better tool of distinguishing protein secondary structure than simple absorption, OA-SFG is expected to be more sensitive to 3D conformational changes in biological macromolecules. This makes it a potentially very useful technique for imaging of biological samples.

More research efforts are clearly needed, however, to press forward chiral SFG microscopy beyond this initial stage of development. When applied to eukaryotic cells, for example, one expects that signals will be complicated by the anisotropic sub-cellular structures in such a way that the polarization selection rules used to isolate the optically active signal no longer hold rigorously. While more research on the sensitivity and selectivity of OA-SFG to probe biological macromolecules is still needed, OA-SFG microscopy, being free from achiral background, has the potential to become a powerful imaging technique for biological samples. 


\subsection{Summary}

In summary, we describe in this chapter the first SFG microscopy that is sensitive to molecular chirality. Using chiral BN solutions as model systems, we demonstrate that our OA-SFG microscope can produce chirality-sensitive images with sub-micron spatial resolution and $3 \mathrm{D}$ sectioning capability. 


\title{
Chapter 6
}

\section{Atomic and molecular parity}

\author{
nonconservation and
}

sum-frequency generation solutions

to the Ozma problem

\subsection{Introduction}

Martin Gardner coined the phrase "Ozma problem" to describe how two advanced civilizations could agree on the definition of left and right without the ability to transmit chiral information, and showed that this cannot be achieved in the absence of parity nonconservation [92]. Gardner and Feynman showed how the Ozma problem could be solved 


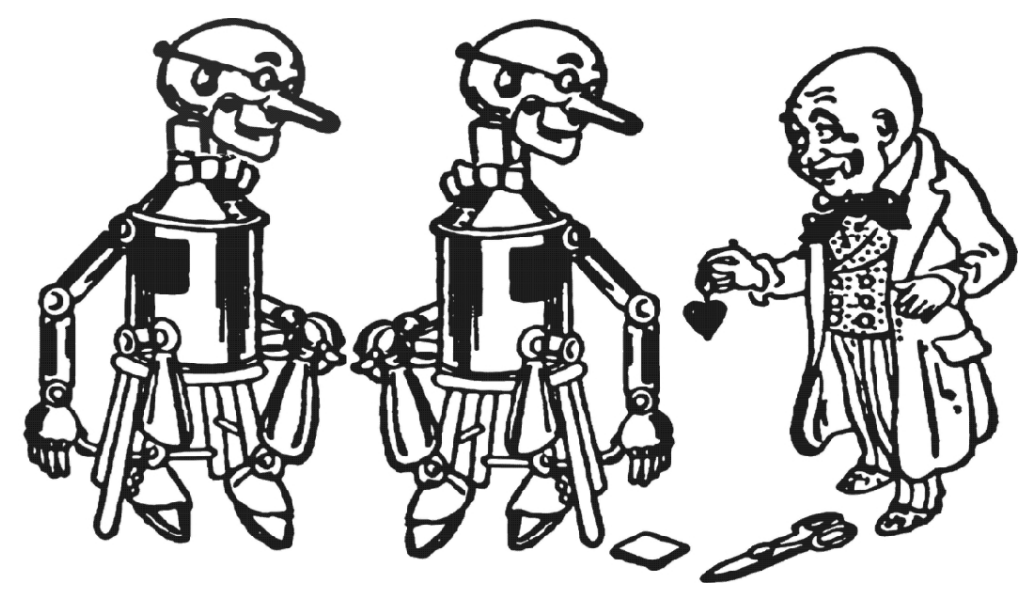

Figure 6.1: Wizard of OZ needs to decide which side of tin woodman's chest to put the heart in. Image adapted from the original drawing by W. W. Denslow in "The Wizard of $\mathrm{Oz} "$

using parity violating aspects of $\beta$-decay $[92,93]$. Atomic optical rotation was proposed as a solution to the problem by Harris [94].

In this chapter we define and solve two Ozma problems. In order to reduce imagery to a minimum we imagine the following scenario, which is taken from the "Wizard of Oz" [95]. A map of $\mathrm{Oz}$ reveals that it is surrounded and separated from Earth by the "Deadly Desert". This desert is assumed to be chirally opaque in that nothing chiral can pass the desert. In the Emerald City of $\mathrm{Oz}$, the Wizard of $\mathrm{Oz}$ is trying to place a heart into the chest of the tin woodman. (See Fig. 6.1.) The Wizard also has a laboratory which analyzes amino acids. We assume the Wizard of $\mathrm{Oz}$ is not from Earth, but from Oz. In addition, Dorothy and Toto are not present, for the presence of creatures from earth would violate the chiral opacity condition. On the earth side of the Deadly Desert an earthly wizard is performing the same heart-implanting operation on a tin woodman. 
Given the above scenario, the Ozma problems are:

The first Ozma problem: Can the two wizards communicate such that the tin woodman will have their hearts placed on the same side, for example, earth's left side?

The second Ozma problem: Suppose both wizards have amino acids which are chemically identical, and of course, chiral. How can the wizards unambiguously transmit the handedness of their amino acid to each other?

In other words, the first Ozma problem is to communicate our definition of left and right. The second Ozma problem is to agree upon the definition of handedness. It is obvious that the solution to the first Ozma problem using $\beta$-decay or atomic optical activity provides the solution to the second Ozma problem. We shall, however, answer these questions using sum-frequency generation (SFG) and parity nonconservation (PNC). SFG provides a new way of exhibiting the interplay of symmetry and parity nonconservation. Indeed, the solution to the second Ozma problem points the way to obtaining, linearly, a parameter which is a manifestation of parity violations in a chiral molecule.

The micro-device built by Skelley et al. is our inspiration for the second Ozma problem [96]. This apparatus is designed to fit on a Martian Rover. Its purpose is to measure net amino acid chirality on Mars. It is a simple leap of the imagination to go from Mars to Oz!

This chapter is organized as follows: After a brief review of SFG, we describe how PNC modifies SFG, and use PNC-modified SFG to answer the Ozma problems. In Appendix B we show another way PNC-modified SFG can measure both chirality and handedness and provide a positive solution to the first Ozma problem. We end the chapter 


\subsection{A BRIEF INTRODUCTION TO SUM-FREQUENCY GENERATION} (SFG)

with a "pedagogical" Feynman joke.

\subsection{A brief introduction to sum-frequency generation (SFG)}

That SFG in three dimensional isotropic chiral media is not zero was proposed by Giordmaine in 1965 [16]. A general theory for SFG was described in Chapter 2. Here we present a simplified version, with the emphasis on its chiral sensitivity.

In an isotropic, homogeneous medium consisting of $N$ identical atoms or molecules, two impinging linearly polarized beams of wave vectors and polarizations $\vec{k}_{1}, \vec{\epsilon}_{1}$ and $\vec{k}_{2}, \vec{\epsilon}_{2}$ result in an outgoing beam, $\vec{k}_{3}, \vec{\epsilon}_{3}$.

In the absence of external fields, and neglecting higher multipoles, the intensity of the outgoing beam for a coherent experiment takes the form,

$$
I\left(\omega_{3}\right) \propto\left(\vec{\epsilon}_{3} \cdot \vec{\epsilon}_{2} \times \vec{\epsilon}_{1}\right)\left|\chi_{\text {chiral }}^{(2)}\left(\omega_{1}, \omega_{2}\right)\right|^{2} .
$$

Here $\chi_{\text {chiral }}^{(2)}\left(\omega_{1}, \omega_{2}\right)$ is a (complex) pseudoscalar and

$$
\chi_{\text {chiral }}^{(2)}\left(\omega_{1}, \omega_{2}\right)=-\chi_{\text {chiral }}^{(2)}\left(\omega_{2}, \omega_{1}\right),
$$

and, dropping the frequency dependence,

$$
\chi_{\text {chiral }}^{(2)}(\mathrm{L})=-\chi_{\text {chiral }}^{(2)}(\mathrm{R})
$$

where $\mathrm{L}$ and $\mathrm{R}$ correspond to left $(\mathrm{L})$ and right $(\mathrm{R})$ handed molecules. $\chi_{\text {chiral }}^{(2)}$ vanishes for achiral molecules, atoms, and racemic mixtures.

The profound thing to notice is that SFG, as described, measures whether a molecule is chiral, but not whether the molecule is L or R. Hence, unlike linear measures of 
chirality, SFG a priori appears useless as a way to answer Ozma problems. However, there are a number of ways to measure the sign of $\chi_{\text {chiral }}^{(2)}$. In particular, three experiments have been carried out to distinguish $\chi_{\text {chiral }}^{(2)}(\mathrm{L})$ and $\chi_{\text {chiral }}^{(2)}(\mathrm{R})$. Two of the experiments involve adding an achiral response to the chiral susceptibility. One method introduces an external electric field [74]. The other uses polarization interferometry in order to add susceptibilities linear in magnetic dipole and electric quadrupole matrix elements [17]. The third method introduces a nonlinear-noncentrosymmetric crystal as a "local oscillator", as described in Chapter 4 [97]. All are germane to the solutions of the Ozma problems.

The knowledge of the sign of $\chi_{\text {chiral }}^{(2)}$ is a necessary but not sufficient condition for the determination of handedness of a chiral molecule. We assume that the additional considerations which relate the sign of $\chi_{\text {chiral }}^{(2)}$ to handedness are available.

\subsection{PNC and SFG}

The manifestations of PNC in atoms and chiral molecules have been reviewed many times with the exception of SFG $[98,99,100]$. Exactly like atomic optical rotation, there is atomic SFG. In the absence of interferometry, SFG intensity of, for example, Thallium (Tl) atoms would be,

$$
I_{\mathrm{Tl}} \sim\left|\chi_{\mathrm{Tl}}^{(2)}\right|^{2}
$$

$\chi_{\mathrm{Tl}}^{(2)}$ is the SFG response of $\mathrm{Tl}$, whose explicit form is irrelevant to what follows. The important point is that $\chi_{\mathrm{Tl}}^{(2)}$, for each pair of input frequencies, has a given sign. This is just like the optical rotation angle, $\theta_{\mathrm{Tl}}$. Because these quantities are manifestations of 
PNC, the opposite signs, $-\chi_{\mathrm{Tl}}^{(2)}$ and $-\theta_{\mathrm{Tl}}$, do not exist. In other words, for given pair of frequencies, $\chi_{\mathrm{Tl}}^{(2)}\left(\omega_{1}, \omega_{2}\right)$ is universal in sign (as long as the universe is made of matter, instead of anti-matter). We shall use this point to solve the Ozma problems.

We now turn to chiral molecules. The presence of PNC is, crudely, akin to adding static electric field with fixed sign. Such a weak electric field generates an achiral response which is added to $\chi_{\text {chiral }}^{(2)}$. PNC is manifested in SFG in the same way. An achiral, or scalar, susceptibility due to PNC is added to the pseudoscalar $\chi_{\text {chiral }}^{(2)}$. To be precise, we define the overall susceptibilities of L- and R- chiral molecules as

$$
\begin{aligned}
\chi^{(2)}(\mathrm{L}) & \equiv \chi_{\text {chiral }}^{(2)}+\Delta \\
\chi^{(2)}(\mathrm{R}) & \equiv-\chi_{\text {chiral }}^{(2)}+\Delta
\end{aligned}
$$

where $\Delta$ is the scalar response due to PNC, and is the same on earth and Oz.

\subsection{Solutions to the second Ozma problem}

We now answer the second Ozma problem in two ways. The first way uses $\chi_{\mathrm{Tl}}^{(2)}$. The second method uses $\Delta$. That is, one way uses $\mathrm{Tl}$ as the source of PNC, the other uses the PNC correction to the $\chi_{\text {chiral }}^{(2)}$ of an amino acid. In both experiments the three polarizations are mutually perpendicular,

$$
\overrightarrow{\epsilon_{3}} \cdot \overrightarrow{\epsilon_{2}} \times \overrightarrow{\epsilon_{1}}= \pm 1
$$

In this configuration achiral and external field contributions to SFG vanish to first order.

In the first experiment we, the wizards on earth, have a gas of $\mathrm{Tl}$ atoms that mixes 
with a gas of amino acid molecules and measure their SFG intensity. On earth we carry out the experiment with $\chi^{(2)}(\mathrm{L})$, say, and obtain a SFG intensity $I_{\mathrm{L}+\mathrm{Tl}}$. We then subtract out the amino acid SFG intensity $I_{\mathrm{L}}$ in the absence of $\chi_{\mathrm{Tl}}^{(2)}$. The result is

$$
I_{\mathrm{L}+\mathrm{Tl}}-I_{\mathrm{L}} \sim 2 \operatorname{Re} \chi_{\mathrm{Tl}}^{(2)} \chi_{\text {chiral }}^{(2) *}
$$

Suppose $\chi_{\mathrm{Tl}}^{(2)}>0$, and $\operatorname{Re} \chi_{\text {chiral }}^{(2)^{*}}$ is greater than zero.

We send $\mathrm{Oz}$ the result of our experiment, which is that the net intensity is greater than 0. If Oz's net intensity is greater than zero, then their amino acid is the same as ours, even if they define handedness differently and call it $\mathrm{R}$. If the net intensity is less than zero, then their amino acid is equivalent to our R, even if they call it L. Hence we have determined the handedness of their amino acid and they have determined ours.

Perhaps there is no $\mathrm{Tl}$ available, then both amino acid enantiomers must be used.

On earth, we obtain for SFG intensities,

$$
\begin{aligned}
& I_{\mathrm{L}} \sim\left|\chi_{\text {chiral }}^{(2)}\right|^{2}+2 \operatorname{Re} \chi_{\text {chiral }}^{(2) *} \Delta+0\left(\Delta^{2}\right) \\
& I_{\mathrm{R}} \sim\left|\chi_{\text {chiral }}^{(2)}\right|^{2}-2 \operatorname{Re} \chi_{\text {chiral }}^{(2) *} \Delta+0\left(\Delta^{2}\right) .
\end{aligned}
$$

Depending on the relative signs of $\Delta$ and $\chi_{\text {chiral }}^{(2)}, I_{\mathrm{L}}$ will either be greater or less than $I_{\mathrm{R}}$. The same obtains on $\mathrm{Oz}$, though what is defined as $\mathrm{L}$ and $\mathrm{R}$ may be reversed. The second Ozma problem is solved.

Interestingly, SFG provides a linear measure of $\Delta$ multiplied by a larger quantity, $\operatorname{Re} \chi_{\text {chiral }}^{(2)^{*}}$. This result is unlike other proposed measurements of PNC which are linear in the parity violating potential $[99,100,101,102]$. Quantitatively, we see that

$$
I_{\mathrm{L}}-I_{\mathrm{R}} \propto 4 \operatorname{Re} \chi_{\mathrm{chiral}}^{(2)^{*}} \Delta .
$$




\subsection{Solutions to the first Ozma problem}

Once both wizards agree on the handedness of an amino acid, many methods are available to solve the first Ozma problem. One is to use optical activity, another is to build a model. We, however, measure all aspects of chirality using SFG. In the absence of Tl we will use one of the now agreed upon amino acid enantiomers. We assume that Wizard of $\mathrm{Oz}$ is to place the tin woodman of Oz's heart on earth's definition of the left side using $\chi^{(2)}(\mathrm{L})$. With that caveat in mind, we use SFG to solve the first Ozma problem.

Both wizards construct an apparatus which we describe using the usual terminology of SFG. The polarizations are defined relative to the plane of the wave vectors $\vec{k}_{1}$ and $\vec{k}_{2}$ (and $\vec{k}_{3}$ by phase matching). Electric field vectors parallel to the plane are defined as $\vec{P}$, those perpendicular are defined as $\vec{S}$. We shall use an external field $\vec{E}$ to supply an extra vector which adds an achiral response to the SFG amplitude.

The plane of wave vectors is taken to be perpendicular to the standing bodies of Wizards and tin woodman. $\vec{k}_{3}$ is in the direction of the tin woodman. The polarizations are $\vec{S}_{1}, \vec{P}_{2}$ and $\vec{P}_{3}$ with $\vec{E}$ perpendicular to the plane of wave vectors and parallel to $\vec{S}_{1}$. We shall consider two configurations on earth, as shown in Fig. 6.2.

Under each experimental configuration, sum-frequency intensity is [74]

$$
I \propto\left|\vec{S}_{1} \cdot \vec{P}_{2} \times \vec{P}_{3} \chi^{(2)}(\mathrm{L})+\left(\vec{S}_{1} \cdot \vec{E}\right) \vec{P}_{2} \cdot \vec{P}_{3} \chi^{(3)}\right|^{2}
$$

where $\chi^{(3)}$ is the achiral response to the $\vec{E}$ field. The two configurations reflect the flipping of wave vectors $\vec{k}_{1}$ and $\vec{k}_{2}$. Note that $\vec{P}_{2}$ and $\vec{P}_{3}$, as defined in Fig. 6.2 , have different directions in configurations 1 and 2. We may write $I_{1}$ and $I_{2}$ in terms of the angle $\theta$ between $\vec{P}_{2}$ and 


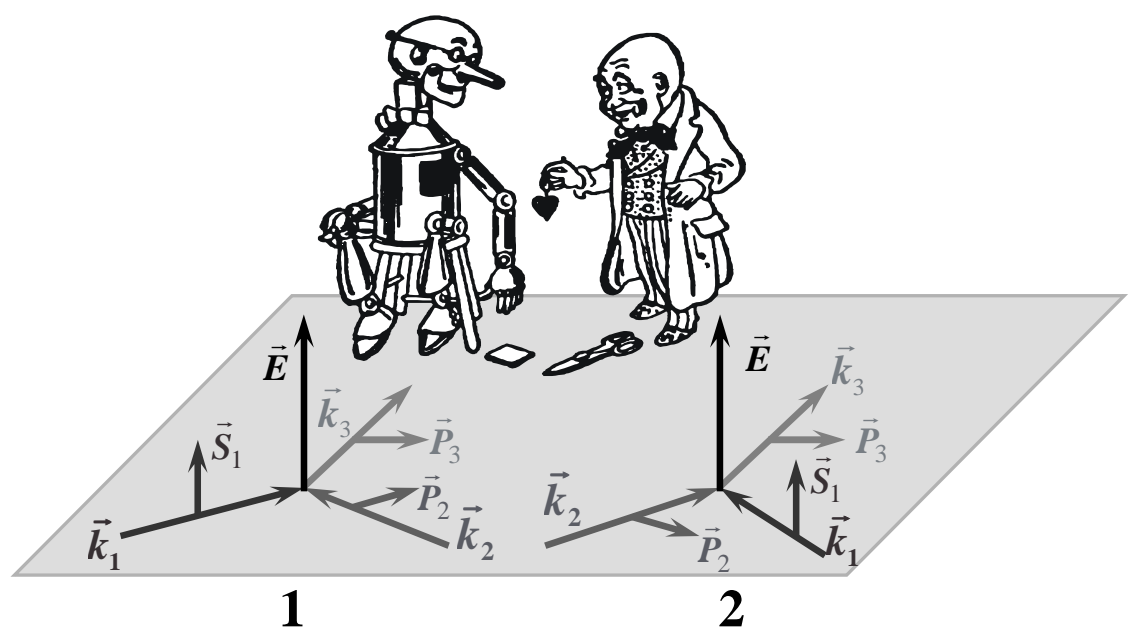

Figure 6.2: Experimental configurations 1 and 2. The electric field $\vec{E}$ is normal to the incident plane defined by the wave vectors $\vec{k}_{1}, \vec{k}_{2}$, and $\vec{k}_{3}$. Polarizations for the three waves are $\vec{S}_{1}, \vec{P}_{2}$ and $\vec{P}_{3}$, respectively, as defined in each configuration. Image adapted from the original drawing by W. W. Denslow in "The Wizard of Oz".

$\vec{P}_{3}$ :

$$
\begin{aligned}
& I_{1} \propto\left|\sin \theta \chi^{(2)}(\mathrm{L})+E \cos \theta \chi^{(3)}\right|^{2} \\
& I_{2} \propto\left|-\sin \theta \chi^{(2)}(\mathrm{L})+E \cos \theta \chi^{(3)}\right|^{2} .
\end{aligned}
$$

Depending on the signs of $\chi^{(2)}(\mathrm{L})$ and $\chi^{(3)}, I_{1}$ will be greater or less than $I_{2}$. Since the amino acid used by the Wizard of $\mathrm{Oz}$ has been determined to be $\mathrm{L}$, then the Wizard can carry out the same two experiments. Because the wave vectors have different frequencies, there will be an unambiguous positioning of left and right. Hence the Wizard of $\mathrm{Oz}$ will be able to place a heart on the tin woodman's left side - the same side as the tin woodman on earth. The first Ozma problem is solved using SFG.

Earlier we described three methods for obtaining the sign of $\chi^{(2)}$. We showed 
how one of the methods, the inclusion of an external electric field, allowed the first Ozma problem to be solved. In Appendix B we carry a complete analysis of a second method that provides an unambiguous solution to the first Ozma problem.

\subsection{Conclusions}

In this chapter we have proposed two Ozma problems using imagery from the "Wizard of Oz". We emphasize that there are many ways to solve the Ozma problems. We have solved both using SFG. The second Ozma problem is, of course, meaningless in the case of atoms. We described two ways of solving the second Ozma problem using SFG and PNC. A byproduct of our solutions is a new way of measuring, linearly, a PNC parameter times a larger quantity.

Our discussions on solving the first Ozma problem, at least partly, depend on equivalent, up to a mirror image, laboratory configurations. We can well imagine experiments as shown in Fig. 6.2 taking place in a spaceship. The results will be the same as long as all relative orientations are unchanged.

Our goal is to provide new "gedanken" solutions to the Ozma problem. We believe that we have succeeded. We have not supplied orders of magnitude of the various responses. They are certainly tiny. As far as we know there have been no calculations of atomic SFG, or the PNC contribution to the SFG of chiral molecules.

Everything in our analysis depends upon $\mathrm{Oz}$ being made of matter rather than antimatter. Our scenario fails in the latter case, as described by a "pedagogical" version of 
a Feynman joke [93]:

Suppose two tin woodmen meet. One is the mirror image of the other.

(i) If P (parity) is conserved and they attempt to shake hands, allow them to do it. Nothing will happen.

(ii) If $\mathrm{P}$ is not conserved, the two tin woodmen will not be strict mirror images.

(iii) If $\mathrm{P}$ and $\mathrm{C}$ (charge conjugation) are not conserved, but $\mathrm{PC}$ is conserved, and one tin woodman puts out his left hand, run! 


\section{Chapter 7}

\section{Sum-frequency vibrational}

\section{spectroscopy of leucine molecules \\ adsorbed at air-water interface}

\subsection{Introduction}

Study of amino acid adsorption at air-water interfaces has two fold significance.

Amino acid molecules are elementary building blocks of proteins and they can appear as anionic, cationic or zwitterionic species depending on the $\mathrm{pH}$ value of the solution. Understanding of their adsorption at air-water interfaces in different charge states can shed light on how various interactions, e.g., electrostatic interaction, Van der Waals interaction, and hydrogen bonding come into play at such interfaces. It can help in search for a better 
understanding of interfacial activities of biomolecules, such as protein folding-unfolding at interfaces [103]. Another aspect of the study is to learn how the interfacial water structure is affected by adsorbed amino acid molecules. Amino acid molecules are expected to adsorb at an air-water interface with their hydrophobic side chain protruding into the air and their hydrophilic terminal into the aqueous solution [104]. They can be considered as a primitive model for studying water structure surrounding protein molecules in physiological conditions that are in a folded configuration with their hydrophilic ends wrapping around the hydrophobic part.

To study adsorption of amino acids at an interface from solution, sum-frequency vibrational spectroscopy (SFVS) is an ideal tool because of its high surface specificity. Watry and Richmond have reported the successful use of the technique to probe adsorption of a number of amino acids at $\mathrm{CCl}_{4}$-water interfaces [105]. However, they have not tried to extract from the spectra detailed information about adsorption characteristics and interfacial structures. In this chapter, we describe our work on the adsorption of amino acid molecules at air-water interfaces, with the emphasis on extracting detailed information from a thorough set of measurements. We chose leucine $\left(\left(\mathrm{CH}_{3}\right)_{2} \mathrm{CHCH}_{2} \mathrm{CH}\left(\mathrm{NH}_{2}\right) \mathrm{COOH}\right)$ as our model amino acid molecules. We studied their adsorption from acidic, near-neutral, and basic solutions of different concentrations, where they behave as cations $\left(\left(\mathrm{CH}_{3}\right)_{2} \mathrm{CHCH}_{2} \mathrm{CH}\left(\mathrm{NH}_{3}^{+}\right) \mathrm{COOH}\right)$, zwitterions $\left(\left(\mathrm{CH}_{3}\right)_{2} \mathrm{CHCH}_{2} \mathrm{CH}\left(\mathrm{NH}_{3}^{+}\right) \mathrm{COO}^{-}\right)$, and anions $\left(\left(\mathrm{CH}_{3}\right)_{2} \mathrm{CHCH}_{2} \mathrm{CH}\left(\mathrm{NH}_{2}\right) \mathrm{COO}^{-}\right)$, respectively, and determined their orientation and surface density in each case. We also observed enhancement and suppression of polar orientation of interfacial water molecules under different $\mathrm{pH}$ conditions. Flipping of the polar orientation occurred when surface charge was 
reversed.

\subsection{Experiment}

Our SFVS setup was described in Chapter 2. The visible input beam at $\omega_{1}$ was fixed at $532 \mathrm{~nm}$ with an intensity of $0.8 \mathrm{~mJ}$ per pulse while the infrared input beam at $\omega_{2}$ was tunable from 2800 to $3800 \mathrm{~cm}^{-1}$ with an energy of $120 \mu \mathrm{J} /$ pulse. Both beams were generated by a picosecond mode-locked Nd:YAG laser pumping an optical parametric system. The input angles of the $\omega_{1}$ and $\omega_{2}$ beams at air-water interfaces were $59^{\circ}$ and $42^{\circ}$, respectively. The reflected SF signal was detected by a gated photo-detection system. A z-cut crystalline quartz plate was used as the reference sample to obtain the absolute value of $\chi_{\mathrm{eff}}^{(2)}$. The leucine aqueous solutions of different bulk concentrations with $\mathrm{pH}$ values of 0.1, 5.9 and 13.1 were prepared. High purity $\mathrm{HCl}$ (20 wt.\% in water, double distilled PPB/Teflon grade, purchased from Sigma-Aldrich, used as received) and $\mathrm{NaOH}$ (pellets, purity 99.998\% purchased from Sigma-Aldrich, used as received) were mixed with ultra-pure water (resistivity $\cong 18.3 \mathrm{M} \Omega$ ) to adjust the $\mathrm{pH}$ values, and checked with SFVS to ensure negligible presence of impurities at surfaces. Racemic leucine used was purchased from Sigma-Aldrich (microselect with purity $\geq 99.5 \%$ ), and used without further purification. In solutions with $\mathrm{pH} \sim 0.1$ (acidic), $\mathrm{pH} \sim 5.9$ (near-neutral), and $\mathrm{pH} \sim 13.1$ (basic), leucine molecules existed as cations, zwitterions and anions, respectively [106]. Due to hydrophobicity of their isopropyl end groups, they tend to adsorb at air-water interfaces with isopropyl groups pointing towards the air. 
Table 7.1: Frequencies, damping constants, and assignment of modes

\begin{tabular}{ccl}
\hline \hline$\omega_{q}\left(\mathrm{~cm}^{-1}\right)$ & $\Gamma_{q}\left(\mathrm{~cm}^{-1}\right)$ & Assignment \\
\hline 2850 & 6 & $\mathrm{CH}_{2}$ symmetric stretch $\mathrm{d}^{+}$ \\
2873 & 5.7 & $\mathrm{CH}_{3}$ symmetric stretch $\mathrm{r}^{+}$ \\
2889 & 7.2 & Fermi resonance of $\mathrm{d}^{+}$or Raman active $\mathrm{d}^{-}$ \\
2902 & 8.0 & $\mathrm{CH}$ symmetric stretch \\
2917 & 7.9 & $\mathrm{CH}_{2}$ asymmetric stretch $\mathrm{d}^{-}$ \\
2938 & 8.3 & Fermi resonance of $\mathrm{r}^{+}$ \\
2953 & 12.0 & $\mathrm{CH}_{3}$ asymmetric stretch $\mathrm{r}_{b}^{-}$: out of plane \\
2963 & 7.8 & $\mathrm{CH}_{3}$ asymmetric stretch $\mathrm{r}_{a}^{-}$: in plane \\
\hline \hline
\end{tabular}

\subsection{Results and discussion}

\subsubsection{Leucine adsorption from acidic solutions}

SFVS spectra of leucine molecules adsorbed at the air-water interface from acidic solutions with different bulk concentrations are shown in Fig. 7.1 for polarization combinations SSP, SPS and PPP. With the increase of bulk concentration, the signal increases. When bulk concentration approaches the saturation value, SFG signal reaches a plateau. We used Eqs. (2.3), (2.4) and (2.18) to fit the spectra. The solid curves in Fig. 7.1 were obtained from the fits. Frequencies and damping constants for different modes deduced from the fits are listed in Table 7.1. They have an accuracy of $\pm 0.5 \mathrm{~cm}^{-1}$.

The observed resonance peaks in the $2800-3000 \mathrm{~cm}^{-1}$ region are due to the $\mathrm{CH}$ stretching modes of leucine molecules. The peaks at $2850 \mathrm{~cm}^{-1}, 2873 \mathrm{~cm}^{-1}, 2902 \mathrm{~cm}^{-1}$, $2917 \mathrm{~cm}^{-1}, 2938 \mathrm{~cm}^{-1}, 2953 \mathrm{~cm}^{-1}$, and $2963 \mathrm{~cm}^{-1}$ can be assigned to the symmetric stretch of the methylene group $\left(\mathrm{d}^{+}\right)$, the symmetric stretch of the two methyl groups $\left(\mathrm{r}^{+}\right)$, the $\mathrm{CH}$ 


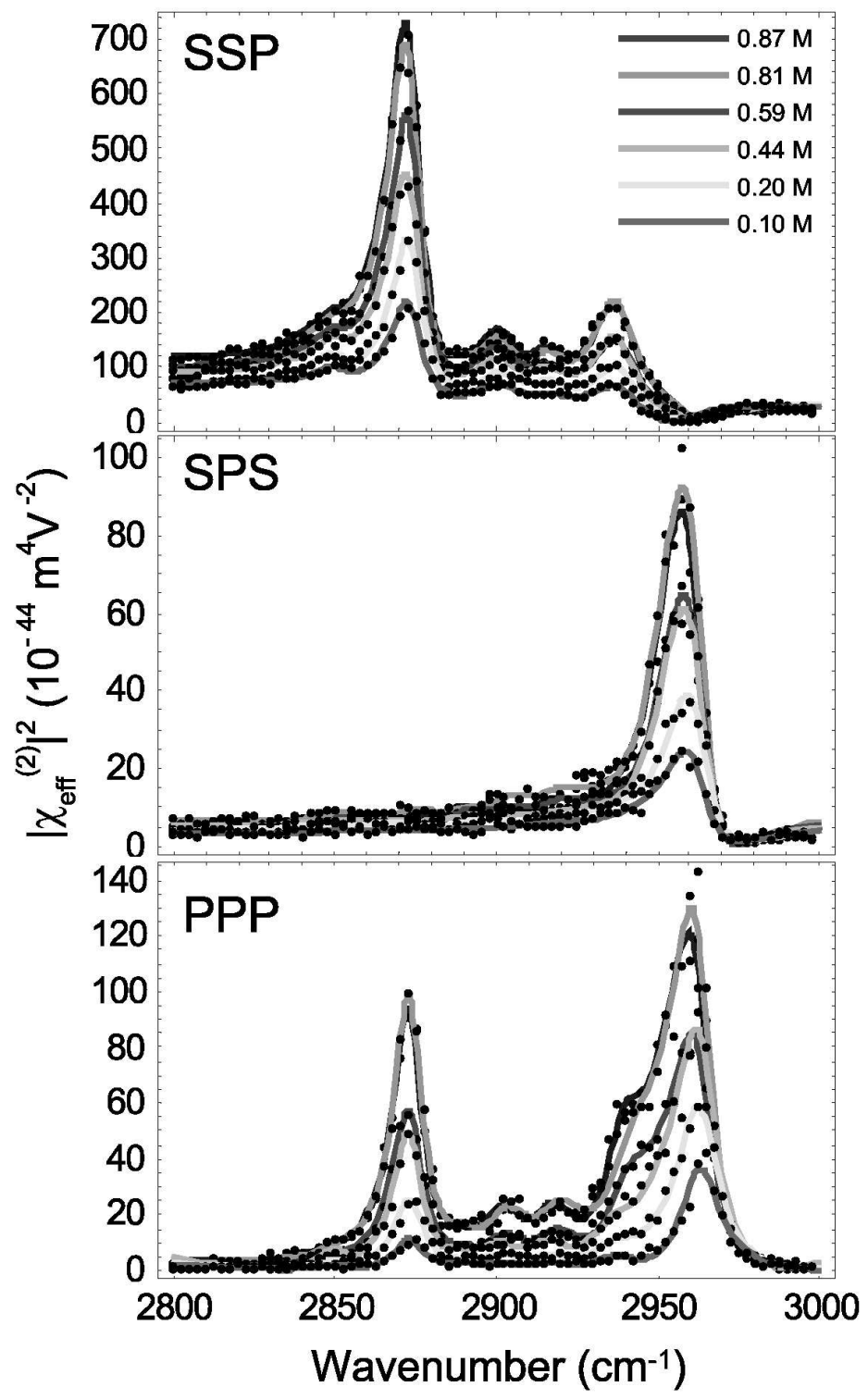

Figure 7.1: SFVS spectra of leucine molecules adsorbed at air-water interface from acidic ( $\mathrm{pH} \sim 0.1)$ solutions with different concentrations. The polarization combinations used were S(SF)S(visible)P(IR), SPS and PPP. Lines are the fitted curves using Eqs. (2.3), (2.4) and $(2.18)$. 
stretch of the methine group, the antisymmetric stretch of the methylene group $\left(\mathrm{d}^{-}\right)$, the Fermi resonance of the methyl symmetric stretch mode $\left(\mathrm{r}_{\mathrm{FR}}^{+}\right)$, and the antisymmetric stretch modes of the methyl groups $\left(\mathrm{r}_{a}^{-}\right.$and $\left.\mathrm{r}_{b}^{-}\right)$, respectively $[107,108,109]$. The small feature at $2889 \mathrm{~cm}^{-1}$ may result from either the Fermi resonance component of $\mathrm{d}^{+}$or the methylene antisymmetric stretch.

The antisymmetric stretch modes $\mathrm{r}_{a}^{-}$and $\mathrm{r}_{b}^{-}$of the methyl groups would be degenerate if the methyl groups were freely rotating. However, in a real environment, the degeneracy is often lifted when the rotation is hindered by interaction with the surrounding, and thus the symmetry of methyl groups is lowered from $\mathrm{C}_{3 \mathrm{v}}$ to $\mathrm{C}_{\mathrm{S}}$. For methyl groups with $\mathrm{C}_{\mathrm{S}}$ symmetry, the transition dipoles for $\mathrm{r}_{a}^{-}$and $\mathrm{r}_{b}^{-}$should be parallel and perpendicular to the symmetry plane, respectively, with $\mathrm{r}_{a}^{-}$being the one with higher frequency. In IR bulk absorption studies, the splitting of these modes has been resolved at room temperature [110]. In surface SFVS, the existence of the non-degenerate antisymmetric stretch modes has been confirmed from fitting and reported in some SFVS studies $[111,112,113]$. The amplitude $\overleftrightarrow{A}_{q}$ 's of these two modes often have opposite signs, which results in a single peak in the intensity profile. For determination of orientations of molecules with a single methyl group, it is often not necessary to analyze the polarization dependence of these antisymmetric modes by simply assuming the methyl group is freely rotating $[114,115,116,117]$. However, as will be shown later, for molecules like leucine with two methyl groups, an analysis on the antisymmetric modes is essential in determining the molecular orientation.

The orientation of the head group of leucine at a surface can be described by three angles, $\theta, \psi$ and $\phi$, as shown in Fig. 7.2, where $\theta$ is the angle between the surface normal $\hat{z}$ 


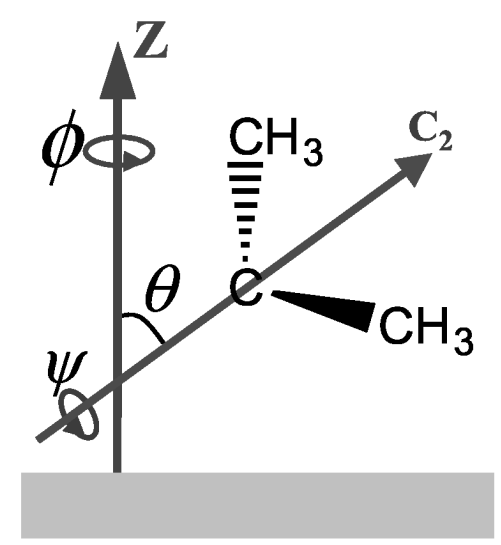

Figure 7.2: Geometry that defines the orientational angles $\phi, \theta$ and $\psi$ of the head groups of leucine molecules $\left(\mathrm{CH}_{3}\right)_{2} \mathrm{CHCH}_{2} \mathrm{CH}\left(\mathrm{NH}_{2}\right) \mathrm{COOH}$ adsorbed at air-water interfaces.

and the $\mathrm{C}_{2}$ symmetric axis of $-\mathrm{C}\left(\mathrm{CH}_{3}\right)_{2}$ group, $\phi$ is the azimuthal angle of the $\mathrm{C}_{2}$ axis on the $\hat{x}-\hat{y}$ plane, and $\psi$ is the "twist" angle between the plane formed by the $\mathrm{C}_{2}$ axis and the surface normal and the plane defined by the three carbon atoms in $-\mathrm{C}\left(\mathrm{CH}_{3}\right)_{2}$. On water surface the leucine monolayer is azimuthally isotropic, therefore $\phi$ is uniformly distributed from 0 to $2 \pi$. For simplicity, we shall assume $\delta$-function distributions for both $\theta$ and $\psi$, and a bond-additive model for calculating the vibrational transition dipole moments and Raman polarizabilities of CH stretch modes in Eq. (2.19) [1, 25, 118].

In principle, we could determine $\theta$ and $\psi$ as well as surface density $N_{\mathrm{S}}$, using Eq. (2.19), from the SFVS measurements of three different polarization combinations, SSP, SPS, and PPP, on the methyl symmetric stretch mode. We found, however, that under our assumptions, the equation for the PPP polarization obtained from Eq. (2.19) is not independent of the equations for SSP and SPS. We need help from measurements of the antisymmetric modes. In our analysis, we took $A_{\mathrm{r}^{+}}(\mathrm{SSP}), A_{\mathrm{r}^{+}}(\mathrm{SPS}), A_{\mathrm{r}_{a}^{-}}(\mathrm{SPS})$ and $A_{\mathrm{r}_{b}^{-}}(\mathrm{SPS})$ 

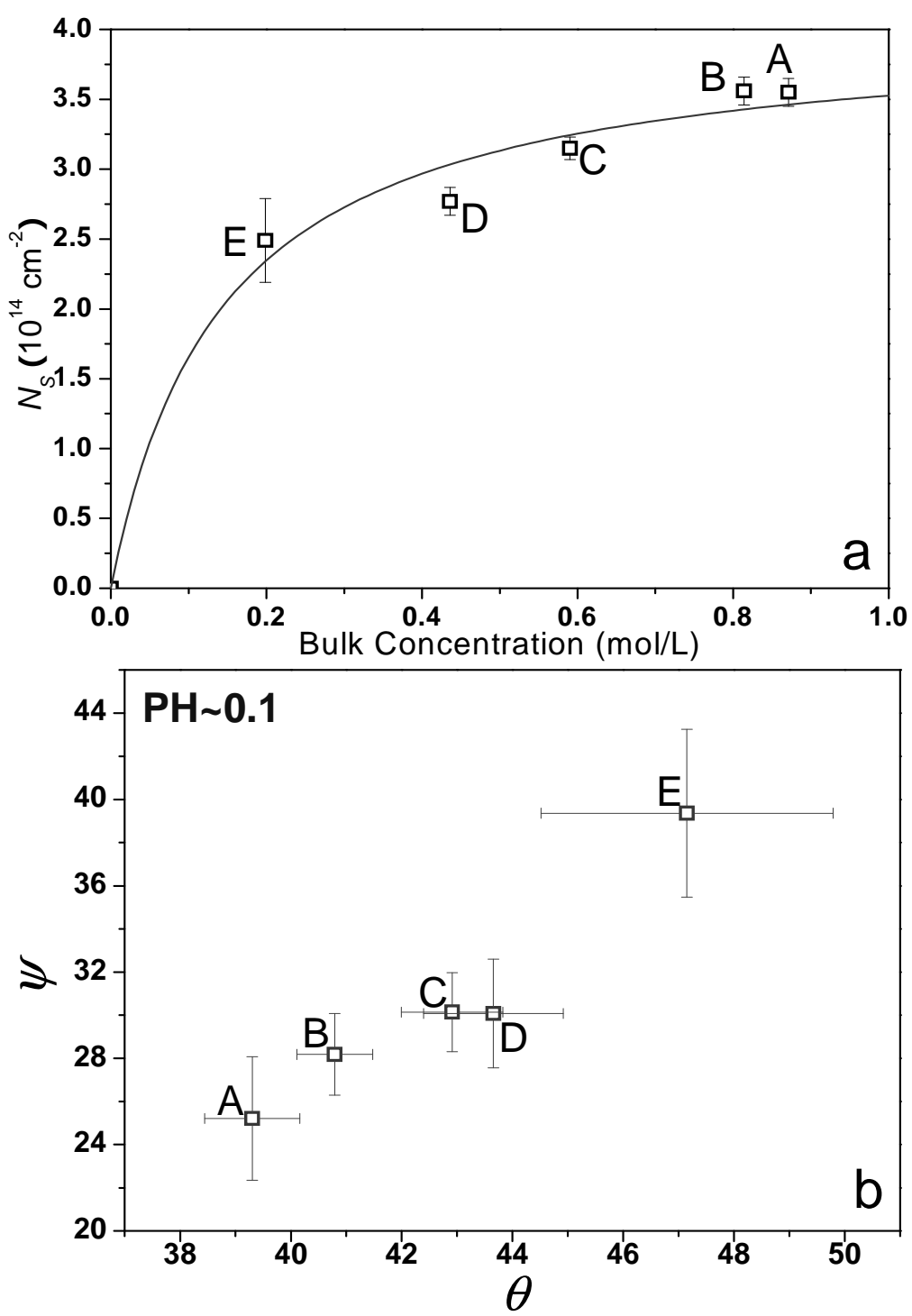

Figure 7.3: (a) Surface density $N_{\mathrm{S}}$ 's versus bulk concentration for acidic solutions. The line is a fitted Langmuir isotherm. (b) Dependence of orientational angles $\theta$ and $\psi$ on surface density for the acidic solution. The surface densities are (A) $3.6 \pm 0.1$, (B) $3.6 \pm 0.1$, (C) $3.2 \pm 0.1,(\mathrm{D}) 2.8 \pm 0.1$, and (E) $2.5 \pm 0.3$ in units of $10^{14} \mathrm{~cm}^{-2}$. 
derived from the experiment to determine the three variables, $\theta, \psi$, and $N_{\mathrm{S}}$, using Eq. (2.19). The values of the other two, $A_{\mathrm{r}_{a}^{-}}(\mathrm{SSP})$ and $A_{\mathrm{r}_{b}^{-}}(\mathrm{SSP})$, are less reliable from the fitting and therefore were ignored in the analysis. ${ }^{1}$ In all cases, we were able to determine first $\theta$ and $\psi$ consistently from the ratios of the four $A$ 's and then $N_{\mathrm{S}}$ from the absolute value of $A_{\mathrm{r}^{+}}(\mathrm{SSP})$ (normalized against crystalline quartz).

The deduced values of $\theta, \psi$, and $N_{\mathrm{S}}$ versus bulk concentration of leucine are plotted in Fig. 7.3 for the acidic solutions $(\mathrm{pH} \sim 0.1)$. As expected, $N_{\mathrm{S}}$ increases with bulk concentration; the dependence can be fitted by a Langmuir isotherm, as shown in Fig. 7.3(a). With increasing surface density, both the tilt angle $\theta$ and the twist angle $\psi$ decreases (Fig. 7.3(b)). This can be understood from consideration that at higher surface densities, interaction between neighboring molecules would squeeze the adsorbed molecules into more upright orientation with $-\mathrm{COOH}$ and $-\mathrm{NH}_{3}^{+}$terminals in water. At the maximum surface density, the molecules in their most stable conformation appear to have $\theta \sim 40^{\circ}$, as seen in the experiment. An optimization that requires the molecules to occupy the smaller surface area could orient the two methyl groups to yield $\psi \sim 25^{\circ}$.

\footnotetext{
${ }^{1}$ In principle, the amplitudes of the two antisymmetric modes in the SSP spectra can also be used in the analysis. However, due to the close proximity of $r_{\mathrm{FR}}^{+}$to these two modes, it is difficult to deduce their amplitudes from the spectra with good accuracy. In the SPS spectra, $\mathrm{r}_{\mathrm{FR}}^{+}$is weak and then the amplitudes of the antisymmetric modes can be reliably determined. In our analysis, we used the four amplitudes that were more reliably determined, two on the antisymmetric modes from the SPS spectra and two on the symmetric mode from the SPS and SSP spectra.
} 
(a) $\mathrm{pH} \sim 5.9$

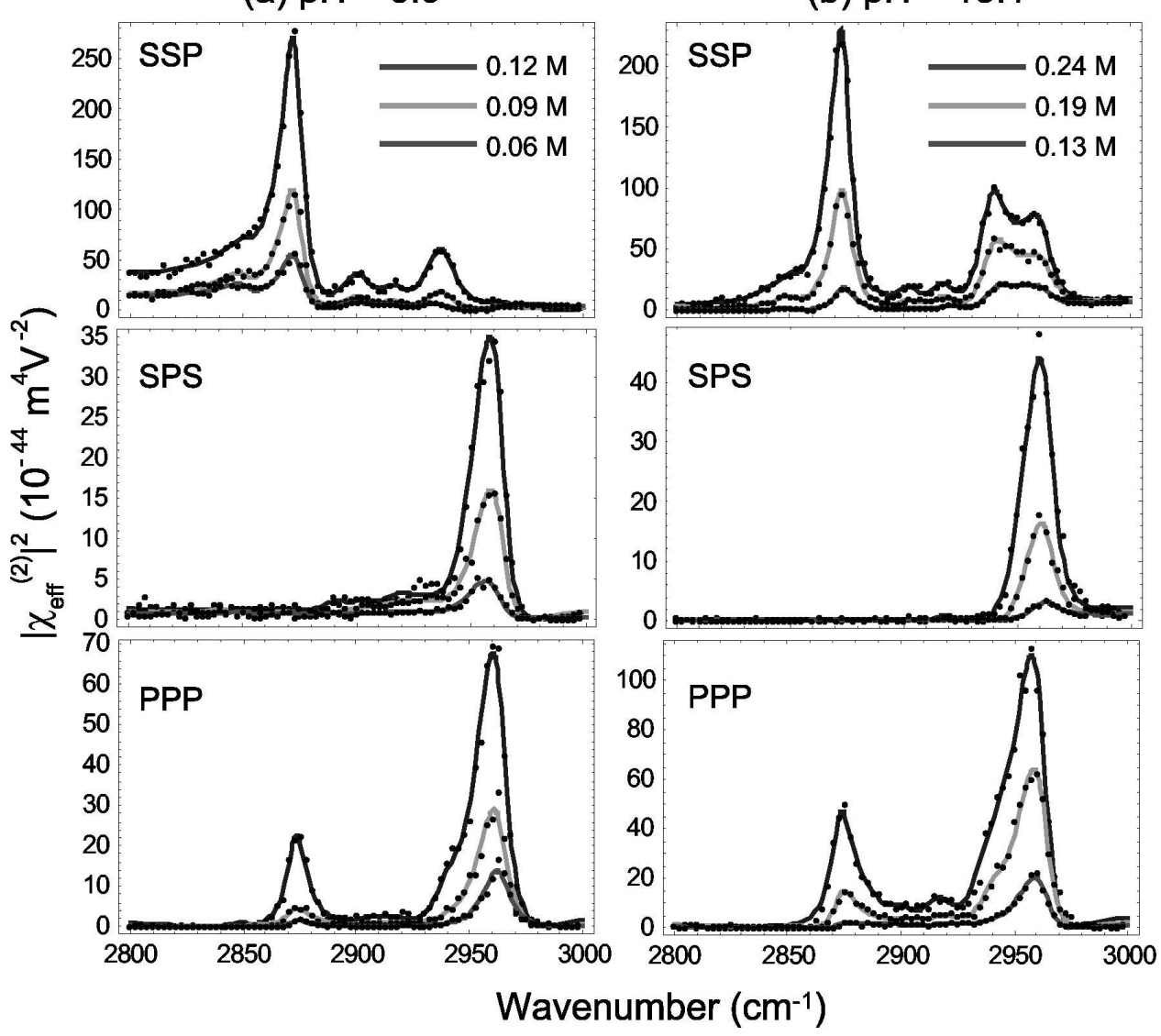

Figure 7.4: SFVS spectra of leucine molecules adsorbed at air-water interface from (a) near-neutral $(\mathrm{pH} \sim 5.9)$ and $(\mathrm{b})$ basic $(\mathrm{pH} \sim 13.1)$ solutions with different concentrations. The polarization combinations used were SSP, SPS and PPP. Lines are fitted curves using Eqs. (2.3), (2.4) and (2.18). 


\subsubsection{Leucine adsorption from near-neutral and basic solutions, and the comparison with acidic case}

We also took SFVS spectra of leucine molecules adsorbed at air-water interfaces from near-neutral ( $\mathrm{pH} \sim 5.9)$ and basic solutions ( $\mathrm{pH}$ 13.1) of a few different bulk concentrations, shown in Fig. 7.4. Qualitatively, they are similar to those from acidic solutions, except that in the SSP spectra around $2960 \mathrm{~cm}^{-1}$ where the two methyl asymmetric stretch modes reside, there is a pronounced peak for the basic solution, a dip for the acidic solution, and a flat line for the near-neutral solution.

The difference is not intrinsic, but results from interference with nonresonant background $\overleftrightarrow{\chi}_{\mathrm{NR}}^{(2)}$ in Eq. (2.18). By choosing appropriate $\overleftrightarrow{\chi}_{\mathrm{NR}}^{(2)}$, we were able to fit all the spectra well using the same set of fitting parameters listed in Table 7.1. To be more explicit, we list in Table 7.2 values of $\overleftrightarrow{\chi}_{\mathrm{NR}}^{(2)}$ around the methyl antisymmetric stretch modes used in our fitting for the three solutions of different $\mathrm{pH}$ values, and show, as examples, in Fig. 7.5 how the SFVS SSP spectra for the three solutions with nearly saturated leucine concentrations appear with and without contribution from $\overleftrightarrow{\chi}_{\mathrm{NR}}^{(2)}$. Without contribution from $\stackrel{\leftrightarrow}{\chi}_{\mathrm{NR}}^{(2)}$, the spectra in the $2960 \mathrm{~cm}^{-1}$ region look very much the same as expected. (We shall discuss later that $\overleftrightarrow{\chi}_{\mathrm{NR}}^{(2)}$ comes from the tail part of the spectral response from the water interfacial layer and why $\overleftrightarrow{\chi}_{\mathrm{NR}}^{(2)}$ for solutions of different $\mathrm{pH}$ values are different.) Using the parameters deduced from fitting, and following the same analytic procedure described earlier, we obtained the values of $N_{\mathrm{S}}$ and orientational angles $\theta$ and $\psi$ for the cases of near-neutral and basic solutions. They are listed in Table 7.3. 
Table 7.2: Real and imaginary part $\left(10^{-22} \mathrm{~m}^{2} \mathrm{~V}^{-1}\right)$ of nonresonant background $\chi_{\mathrm{NR}}^{(2)}$ at 2960 $\mathrm{cm}^{-1}$ obtained from fitting for SSP spectra of three near-saturated solutions with different $\mathrm{pH}$ values (Both the real and imaginary part are assumed to change linearly with infrared wavenumber in the range $2800-3000 \mathrm{~cm}^{-1}$.)

\begin{tabular}{lcc}
\hline \hline Solution & $\operatorname{Re} \chi_{\mathrm{NR}}^{(2)}$ & $\operatorname{Im} \chi_{\mathrm{NR}}^{(2)}$ \\
\hline Acidic 0.87M & $-7.46 \pm 0.73$ & $-2.02 \pm 0.52$ \\
Near-neutral 0.12M & $-2.22 \pm 0.37$ & $0.62 \pm 0.68$ \\
Basic 0.24M & $-1.17 \pm 0.32$ & $2.43 \pm 0.27$ \\
\hline \hline
\end{tabular}
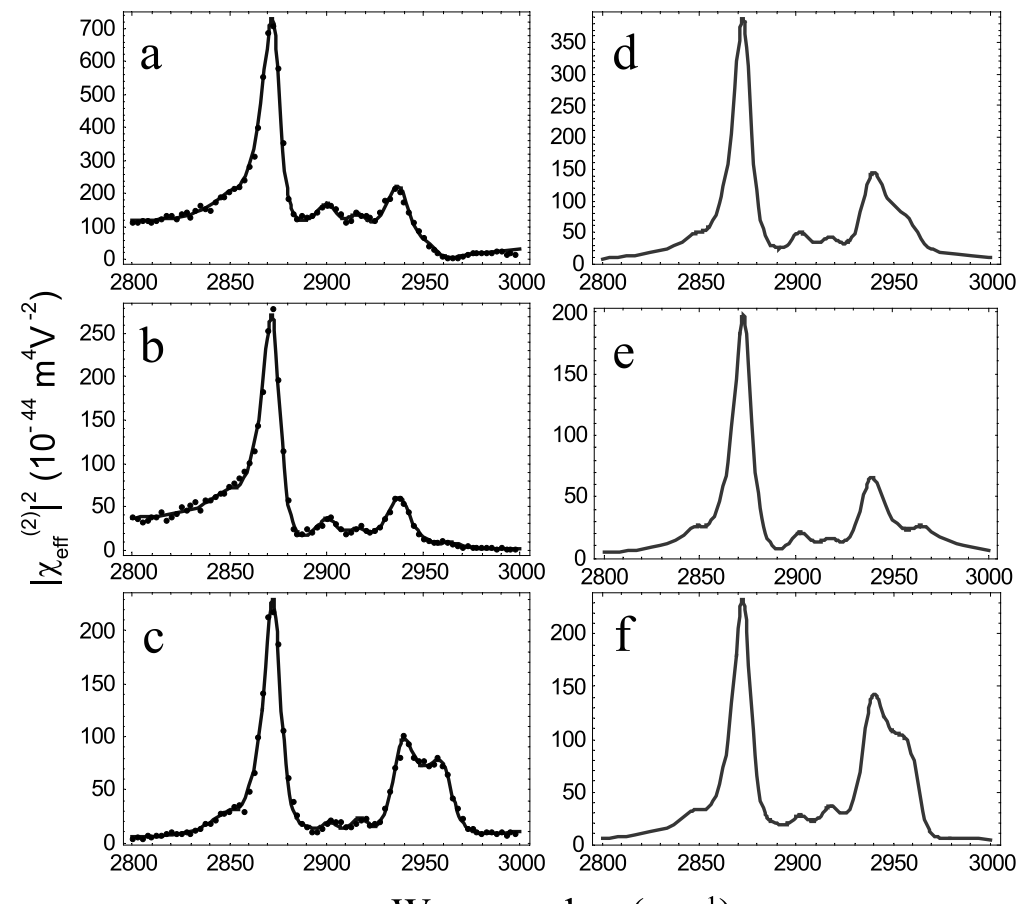

Wavenumber $\left(\mathrm{cm}^{-1}\right)$

Figure 7.5: SFVS spectra of leucine molecules adsorbed from nearly saturated (a) acidic, (b) near-neutral and (c) basic solutions, together with the simulated SFVS spectra that are free of the nonresonant background for (d) acidic, (e) near-neutral and (f) basic solutions. Polarization combination used was SSP. 
Table 7.3: Deduced orientational angles $\theta$ and $\psi$ for leucine molecules adsorbed at air-water interfaces from near-neutral and basic solutions

\begin{tabular}{cccc}
\hline \hline \multicolumn{4}{c}{$\mathrm{pH} \sim 5.9$} \\
$\mathrm{C}(\mathrm{mol} / \mathrm{L})$ & $N_{\mathrm{S}}\left(10^{14} \mathrm{~cm}^{-2}\right)$ & $\theta$ (degree) & $\psi$ (degree) \\
\hline 0.06 & $1.1 \pm 0.1$ & $40 \pm 4$ & $26 \pm 6$ \\
0.09 & $1.9 \pm 0.1$ & $43 \pm 2$ & $26 \pm 3$ \\
0.12 & $2.5 \pm 0.1$ & $39 \pm 1$ & $21 \pm 3$ \\
\hline \multicolumn{4}{c}{$\mathrm{pH} 13.1$} \\
$\mathrm{C}(\mathrm{mol} / \mathrm{L})$ & $N_{\mathrm{S}}\left(10^{14} \mathrm{~cm}^{-2}\right)$ & $\theta$ (degree) & $\psi$ (degree) \\
\hline 0.13 & $1.3 \pm 0.2$ & $53 \pm 6$ & $43 \pm 5$ \\
0.19 & $1.8 \pm 0.2$ & $43 \pm 6$ & $22 \pm 5$ \\
0.24 & $2.7 \pm 0.1$ & $39 \pm 1$ & $17 \pm 3$ \\
\hline \hline
\end{tabular}

Same as the acidic solution, the surface density of leucine molecules, $N_{\mathrm{S}}$, increases with the bulk concentration for near-neutral and basic solutions. However, to yield the same surface density, the leucine bulk concentration in the near-neutral solution is the lowest, that is, leucine is most surface-active in near-neutral solutions in which the leucine molecules should appear zwitterionic. This agrees with general observation for ampholytic surfactants in aqueous solutions that they are most surface-active in the zwitterionic form and become less so when they are converted to anionic or cationic form $[119,120]$. The reason is that in the zwitterionic form, their interaction with water compared to the interaction among themselves is the weakest, and therefore, their solubility in water is the lowest. For acidic and basic solutions, the additional ions from $\mathrm{HCl}$ and $\mathrm{NaOH}$ in water can screen the electrostatic repulsion between anionic or cationic leucine molecules (Debye-Hückel screening), thus increasing the solubility and stabilizing the charge accumulation both in the solution and at the air-water interface. As a result, acidic and basic solutions have higher bulk 
and surface saturation densities. In our experiment, the acidic solution ( $\mathrm{pH} 0.1$ ) has the highest saturation densities because of the highest ionic strength. The adsorption behavior we observed for the three solutions with different $\mathrm{pH}$ values agrees qualitatively with the result obtained from a pH-dependent surface tension study by Wustneck et al on ampholytic surfactants [119].

The orientational angles $\theta$ and $\psi$ for leucine adsorbed at air-water interfaces of near-neutral and basic solutions also seem to decrease with the increase of surface density as in the case of acidic solutions, although our data at lower surface densities are limited because of the poorer spectral quality. At their maximum surface density, the molecular orientation again has $\theta \sim 40^{\circ}$, but the twist angle $\psi$ for acidic, near-neutral, and basic solutions are around $25^{\circ}, 21^{\circ}$, and $17^{\circ}$. This is probably the result of how the hydrophilic terminals of the adsorbed molecules interact with water. In the near-neutral solution, the $\mathrm{NH}_{3}^{+}$and $\mathrm{COO}^{-}$terminals anchor evenly in water, and the corresponding optimal molecular orientation supposedly is specified by $\theta \sim 40^{\circ}$ and $\psi \sim 21^{\circ}$. In the acidic solution, the $\mathrm{NH}_{3}^{+}$ terminal anchors more strongly in water and the $\mathrm{COOH}$ terminal could be somewhat lifted to optimize the overall molecular orientation. This would lead to a larger $\psi$ with little change in $\theta$. On the other hand, in the basic solution, the $\mathrm{COO}^{-}$terminal anchors more strongly in water and the $\mathrm{NH}_{2}$ terminal could be somewhat lifted to yield a smaller $\psi$. A molecular dynamics simulation would be needed to confirm the above picture. 

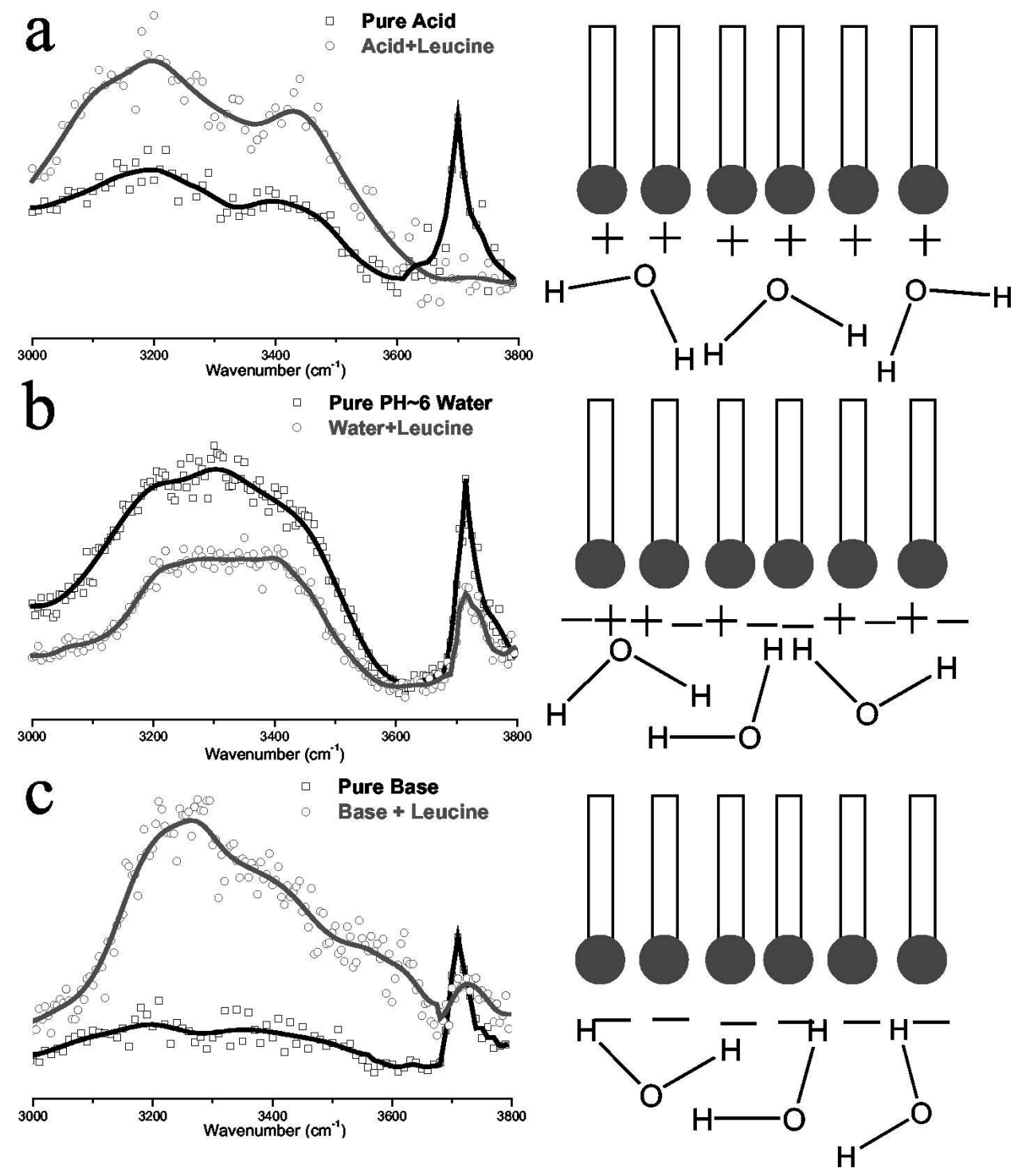

Figure 7.6: SFVS spectra of water at air-water interfaces with and without adsorbed leucine molecules. The bulk $\mathrm{pH}$ values of the solutions are $(\mathrm{a}) \sim 0.1,(\mathrm{~b}) \sim 5.9$, and $(\mathrm{c}) \sim 13.1$. Schematics on the side depict the interfacial water structure upon the adsorption of leucine molecules. The circles stand for the hydrophilic part of leucine molecules, and the rectangles depict the hydrophobic part. Polarization combination used was SSP. The solid lines are guides to the eye. 


\subsubsection{Interfacial water structure probed with SFVS}

Adsorbed leucine monolayers could alter the interfacial water structure. To investigate such an effect, we recorded SFVS spectra of $\mathrm{OH}$ stretch modes in the $3000 \mathrm{~cm}^{-1}$ to $3800 \mathrm{~cm}^{-1}$ range for air-water interfaces of leucine solutions with SSP polarization combination. The spectra from near-saturated acidic, near-neutral and basic solutions of leucine molecules are presented in Fig. 7.6, in comparison with spectra from solutions without leucine.

Although quantitative analysis on these spectra is complicated by possible contribution from $\mathrm{NH}$ stretching modes of the adsorbed leucine molecules in this range, a qualitative discussion on several observations could be informing. ${ }^{2}$ For acidic and basic leucine solutions, the spectra in the bonded $\mathrm{OH}$ range $\left(3000 \sim 3600 \mathrm{~cm}^{-1}\right)$ are stronger than the corresponding ones without leucine. The so-called ice-like peak around $3200 \mathrm{~cm}^{-1}$ also appears relatively stronger. In these cases, the air-water interfaces of the acidic and basic solutions are positively and negatively charged, respectively, by the adsorbed cationic and anionic leucine molecules. It is known that the surface field created by the surface charges can induce more polar ordering as well as ordering of the hydrogen-bonding network in the interfacial water layer [122]. This is reflected by the overall stronger spectrum and stronger ice-like peak as we have observed. For the near-neutral solution, the spectrum with leucine is weaker than the one without leucine and can be explained by the presence of a more disordered water interfacial layer. Since the adsorbed leucine molecules at the interface is

\footnotetext{
${ }^{2}$ SFVS results from ammonia monolayers on water and ice suggest that the NH stretch modes are several times weaker than the $\mathrm{OH}$ stretch modes of water in the spectra [121].
} 
in the zwitterionic form, with the amino group positively charged and the carboxy group negatively charged (shown in Fig. 7.6(b)), water molecules in close proximity orient differently according to their local charge or field conditions. This results in a more disordered structure at the interface as compared with that of a free water surface, and hence a weaker SFVS spectrum.

The above picture also suggests that with the surface charges being positive or negative for the acidic or basic solutions, the interfacial water molecules must have a net dipole orientation pointing toward or away from the interface. This allows us to answer the earlier question why the imaginary part of the $\stackrel{\leftrightarrow}{\chi}_{\mathrm{NR}}^{(2)}$ 's deduced from fitting of the spectra in Fig. 7.5 and listed in Table 7.2 has different signs for acidic and basic leucine solutions and very weak for the near-neutral solution. ${ }^{3}$ The nonresonant background in the $\mathrm{CH}$ stretch region comes from the extended tail of the ice-like peak of water, and the imaginary part of $\overleftrightarrow{\chi}_{\mathrm{NR}}^{(2)}$ must change sign if the orientation of water molecules flips. Its magnitude depends on the strength of the ice-like peak, and is larger for acidic and basic cases, due to better polar ordering of the interfacial water molecules.

The picture that the water molecules at a charged surface have a preferred dipole orientation toward or against the surface is also supported by the behavior of the free $\mathrm{OH}$ stretching mode at $3700 \mathrm{~cm}^{-1}$. As seen in Fig. 7.6, this mode is present at all interfaces except the one associated with the acidic leucine solution. In the latter case, the surface field must have oriented the surface molecules with their dipoles pointing toward the solution

\footnotetext{
${ }^{3}$ The real part of $\overleftrightarrow{\chi}_{\mathrm{NR}}^{(2)}$ comes from all nonresonant transitions, while the imaginary part of $\overleftrightarrow{\chi}_{\mathrm{NR}}^{(2)}$ comes dominantly from the neighboring near-resonant modes. Thus the imaginary part of $\overleftrightarrow{\chi}_{\mathrm{NR}}^{(2)}$ better reflects the neighboring modes.
} 
and therefore eliminated the free $\mathrm{OH}$ bonds protruding out of water. Related to the water structure surrounding folded protein molecules on which there are both positive and negative charges, our study here suggests that it can be quite significantly disordered depending on the local charge distribution.

\subsection{Conclusion}

In conclusion, we have used SFVS to study adsorption of leucine molecules at the air-water interface from solutions with different concentrations and $\mathrm{pH}$ values. The symmetric and antisymmetric stretch modes of methyl group with their polarization dependence were used to deduce the orientation of adsorbed molecules. The adsorption isotherm of leucine from acidic solution was obtained. It was found that at the saturated surface density, the adsorbed molecules take on an upright position, which changes only slightly for molecules in different charge states. Interfacial water structure, however, can be strongly perturbed by the charge state. Both the polar order and the hydrogen-bonding order of interfacial water molecules are enhanced when the surface carries uniform charges, but suppressed when both negative and positive charges are simultaneously present at the interface. The polar orientation is inverted when the solution changes from acidic to basic. 


\title{
Chapter 8
}

\section{Surface vibrational spectroscopy}

\author{
on shear-aligned
}

\section{poly(tetrafluoroethylene) films}

\subsection{Introduction}

Poly(tetrafluoroethylene) (PTFE, $\mathrm{C}_{n} \mathrm{~F}_{2 n}$ ) is well known for its remarkable surface properties. It is used to coat cookwares to make them nonstick and to make fabric waterrepellant. Recently it was found that highly oriented PTFE thin films could be rubbed onto a substrate by shearing of a hot piece of PTFE. They can be used as templates to orient biological molecules [123], organic films and crystals, as well as polymers deposited on them [124]. Various mechanisms such as epitaxy [125], graphoepitaxy [126], and to- 
pographical induction [127] have been proposed to explain the surface-induced ordering effect. Clearly, for basic understanding of the effect, one needs to know the surface structure of the films at the molecular level. Transmission electron microscopy, X-ray diffraction, atomic force microscope, and high-resolution electron energy loss spectroscopy have been used to study such films, but they are generally not sufficiently surface-specific or sensitive to provide much detailed information about the surface structure [128].

In this context, sum-frequency vibrational spectroscopy (SFVS) is an ideal tool. It is highly surface-specific and sensitive to a surface monolayer. The polarization-dependent surface vibrational spectra allow deduction of information about orientations of different functional groups. The technique has been successfully employed to probe surfaces of polymers with stretch modes in the $\mathrm{CH}$ and $\mathrm{CO}$ ranges [129]. In this communication, we report our study of surface structure of shear-deposited PTFE films using SFVS. The observed PTFE spectra are the first surface vibrational spectra ever obtained in the CF stretch region. From the spectra obtained with different sample orientations and input-output polarization combinations, we find that the PTFE chains are well aligned on the surface along the shearing direction. With the help of selection rules, the vibrational spectra associated with the well-aligned PTFE chains enable us to properly identify the vibrational modes of PTFE, helping to resolve the long-standing controversy over their assignment $[130,131]$. 


\subsection{Experimental results}

The PTFE thin films were prepared by shearing a hot PTFE rod at $300^{\circ} \mathrm{C}$ against a clean fused silica substrate at $200^{\circ} \mathrm{C}$ under constant pressure $\left(\sim 5 \times 10^{4} \mathrm{~Pa}\right)$ and constant speed $(\sim 0.5 \mathrm{~cm} / \mathrm{s})$. They appeared to SFVS to have forward-backward symmetry along the shearing direction and hence the $\mathrm{C}_{2 \mathrm{~V}}$ symmetry. Defining $\mathrm{Z}$ to be along the surface normal and $\mathrm{X}$ along the shearing direction, we have the following nonvanishing surface susceptibility elements for the films [35]:

$$
\chi_{\mathrm{XXZ}}^{(2)}, \chi_{\mathrm{YYZ}}^{(2)}, \chi_{\mathrm{ZZZ}}^{(2)}, \chi_{\mathrm{XZX}}^{(2)} \cong \chi_{\mathrm{ZXX}}^{(2)}, \chi_{\mathrm{YZY}}^{(2)} \cong \chi_{\mathrm{ZYY}}^{(2)}
$$

The experimental arrangement was similar to the one described in Chapter 7. A visible beam at $532 \mathrm{~nm}$ and an infrared beam tunable from 750 to $1300 \mathrm{~cm}^{-1}$, generated from a picosecond Nd:YAG laser system and an optical parametric system, were overlapped on a PTFE film exposed to air. The sum-frequency signal in reflection was detected by a photodetector/gated integrator system after proper spatial and spectral filtering. The SFVS spectra were taken with the film so oriented that the shearing direction was either parallel $(/ /)$ or perpendicular $(\perp)$ to the plane of incidence. Different polarization combinations were used: SSP to deduce $\chi_{\mathrm{XXZ}}^{(2)}$ and $\chi_{\mathrm{YYZ}}^{(2)}$, SPS to deduce $\chi_{\mathrm{XZX}}^{(2)}$ and $\chi_{\mathrm{YZY}}^{(2)}$, and PPP to deduce the remaining $\chi_{\mathrm{ZZZ}}^{(2)}$. The spectra appeared to be remarkably simple. No spectral features were observed in any polarization combination except SPS, in which two peaks at $\omega_{q}$ of 1142 and $1204 \mathrm{~cm}^{-1}$ and $\Gamma_{q}$ of 5 and $12 \mathrm{~cm}^{-1}$, respectively, appeared when the sample was in the // geometry, as shown in Fig. 8.1.

The shear-deposited films had an average thickness of $~ 10 \mathrm{~nm}$ seen by AFM [128, 


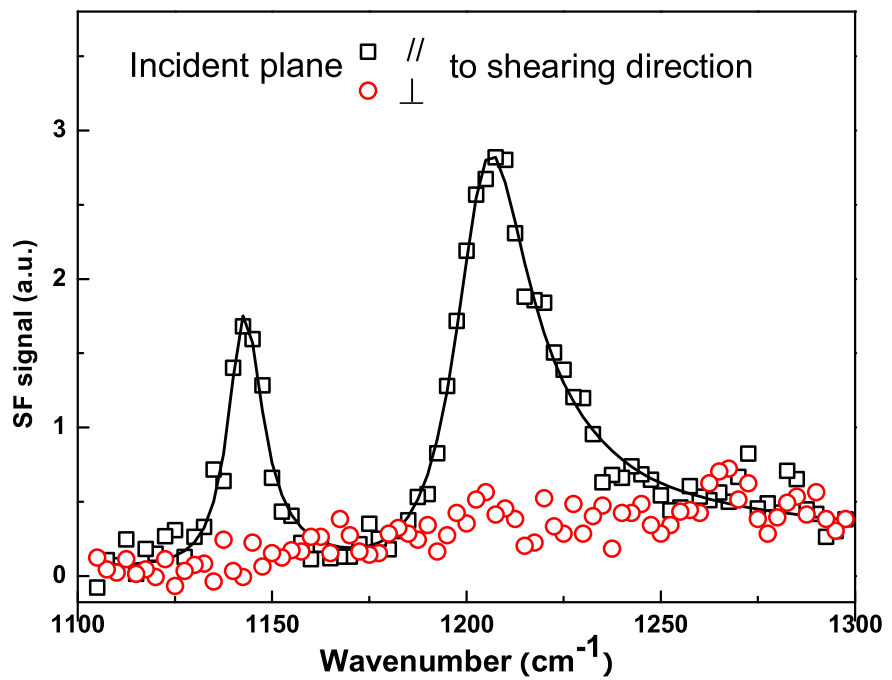

Figure 8.1: SFVS spectra from a shear-deposited thin film of PTFE. The polarization combination used was $\mathrm{S}(\mathrm{SFG}) \mathrm{P}($ Visible)S(Infrared).

130, 132]. One might wonder if the PTFE bulk or PTFE/substrate interface would contribute to the observed SPS spectrum. As a test, we covered the PTFE surface by a $\mathrm{BaF}_{2}$ window with a thin layer of $\mathrm{CCl}_{4}$ in between. The $\mathrm{CCl}_{4}$ layer had no absorption in the $1100-1300 \mathrm{~cm}^{-1}$ region, and yet the SFVS spectrum disappeared completely. It reappeared immediately after evaporation of $\mathrm{CCl}_{4}$. Apparently the $\mathrm{CCl}_{4}$ liquid must have varied the local environment of PTFE and suppressed its SFVS spectrum. This indicates that the sum-frequency signal originated from the air/PTFE interface. 


\subsection{Discussion}

To understand the SFVS spectra and deduce information about the surface structure of the PTFE films, we follow the theoretical studies of vibrational modes of PTFE in the literature $[133]$. PTFE is a linear polymer of $\left[-\left(\mathrm{CF}_{2}\right)-\right]_{n}$. Unlike polyethylene $\left(\mathrm{C}_{n} \mathrm{H}_{2 n}\right)$ which has a planar zigzag structure, PTFE chains have a $15_{7}$ helical structure under room temperature in which $15 \mathrm{CF}_{2}$ groups are arranged along the helical axis in seven turns. ${ }^{1}$ Coupling between $\mathrm{CF}_{2}$ groups along a chain is also stronger than $\mathrm{CH}_{2}$, leading to normal vibrational modes that cannot be identified with individual $\mathrm{CF}_{2}$ groups. Factor group analysis [134] for such a structure shows that its normal modes must have $A_{1}, A_{2}, E_{1}$ or $\mathrm{E}_{2}$ symmetry; only the $\mathrm{E}_{1}$ modes are both infrared and Raman active and their infrared transition dipole is perpendicular to the helical axis.

As shown in Eq. (2.19), SFVS requires the vibrational modes to be both infrared and Raman active. Thus only modes with $\mathrm{E}_{1}$ symmetry can be detected. To excite the $\mathrm{E}_{1}$ modes, one needs an infrared field component along the transition dipole. Now in our SFVS experiment on PTFE, vibrational modes were detected only with the SPS polarization combination and the // geometry. Therefore, they must have their transition dipoles perpendicular to the shearing direction of the film. This then strongly suggests the following scenario. The PTFE chains must be well aligned on the surface in the shearing direction as depicted in Fig. 8.2(knowing that the bulk PTFE chains are well oriented by shearing of the film $[128,130,132])$, and the vibrational modes observed in SFVS are of the $\mathrm{E}_{1}$ symmetry. No other reasonable models can explain the experimental observation equally satisfactorily.

\footnotetext{
${ }^{1}$ Although each helix is chiral, the films are racemic with equal number of right- and left-handed helices.
} 


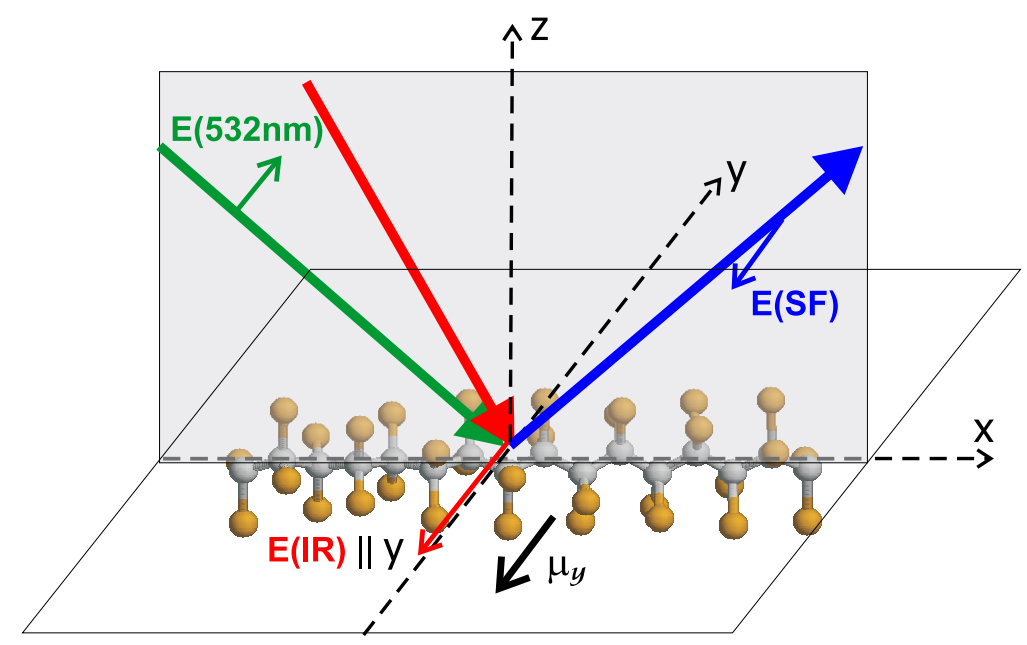

Figure 8.2: Schematic showing a PTFE chain aligned along the shearing direction in the incident plant. Electric field directions of the beams as marked are of SPS polarization combination.

The fact that the vibrational modes only appear in the SPS and // configuration indicates that among the five independent $\chi_{i j k}^{(2)}$ elements allowed by surface symmetry of the PTFE film, only $\chi_{\mathrm{YZY}}^{(2)} \cong \chi_{\mathrm{ZYY}}^{(2)}$ are actually nonvanishing. This must result from intrinsic symmetry of the PTFE structure. That the vibrational modes have $\mathrm{E}_{1}$ symmetry and the chains are aligned along the shearing direction makes $\chi_{\mathrm{XZX}}^{(2)} \cong \chi_{\mathrm{ZXX}}^{(2)}$ vanish, but we still need to explain why $\chi_{\mathrm{XXZ}}^{(2)}, \chi_{\mathrm{YYZ}}^{(2)}$, and $\chi_{\mathrm{ZZZ}}^{(2)}$ also nearly vanish. It can be shown [133] from group theory that for $\mathrm{E}_{1}$ modes of a PTFE chain in space with $15_{7}$ symmetry, the only allowed Raman polarizability elements are $\alpha_{y x}=\alpha_{x y}$ and $\alpha_{z x}=\alpha_{x z}$, where the molecular coordinate $x$ is defined to be along the axis of the helical chain. Having the chain oriented along $\mathrm{X}$ ( $\operatorname{such}$ that $x=\mathrm{X}$ ), we must conclude that $\chi_{\mathrm{XXZ}}^{(2)}, \chi_{\mathrm{YYZ}}^{(2)}, \chi_{\mathrm{ZZZ}}^{(2)}$, as well as $\chi_{\mathrm{YZY}}^{(2)}$ must all vanish. However, for surface PTFE chains, the symmetry breaking of $\mathrm{Z}$ versus $-\mathrm{Z}$ makes 
$\alpha_{y z}=\alpha_{z y}$ allowed..$^{2}$ In this case, $\chi_{\mathrm{YZY}}^{(2)}$ appears to be the only nonvanishing $\overleftrightarrow{\chi}^{(2)}$ element as we have observed.

That we can conclude the observed PTFE vibrational modes at 1142 and 1204 $\mathrm{cm}^{-1}$ are of the $\mathrm{E}_{1}$ symmetry is interesting and meaningful. Ever since the early investigation nearly 50 years ago, the assignment of PTFE's vibrational modes has always been a matter of debate. IR and Raman spectroscopy on bulk PTFE generally reveal three modes around 1150,1200 , and $1240 \mathrm{~cm}^{-1}$ in the CF stretch range, but their assignment varies among different authors [130, 131]. In our SFVS spectrum, the $1240 \mathrm{~cm}^{-1}$ mode is very weak and hardly distinguishable from the background noise. It indicates that the mode must be of symmetry other than $\mathrm{E}_{1}$. Our results agree with the earlier assignment of this mode to be of $\mathrm{A}_{2}$ symmetry by Peacock et al [135] and Cutler et al [136].

\subsection{Conclusion}

In conclusion, we have used SFVS to obtain the first surface vibrational spectra of PTFE films. The surprisingly simple spectra for the sheared films enable us to conclude that the helical PTFE chains are well aligned on the surface along the shearing direction, and the observed vibrational modes at 1142 and $1204 \mathrm{~cm}^{-1}$ are of the $\mathrm{E}_{1}$ symmetry in agreement with some earlier analysis.

\footnotetext{
${ }^{2}$ This is similar to the differences between $\mathrm{C}_{2 \mathrm{~V}}$ and $\mathrm{C}_{2}$ symmetry.
} 


\section{Chapter 9}

\section{Conclusion}

Since sum-frequency generation (SFG) was demonstrated as an electric-dipole allowed tool to detect chirality in 2000 , it has been used to measure optically active vibrational, electronic, and vibration-electronic double-resonance spectra. This thesis describes our recent progresses in its development.

As a first step toward using SFG as a spectroscopic tool for biological analysis, we demonstrated that SFG can provide optically active electronic spectra of chiral amino acids in solution. To explain the observed chiral signal near the electronic resonances of the intrinsically achiral chromophores, we developed a dynamic coupling model that gives an analytical expression for $\chi_{\text {chiral }}^{(2)}$. The chiral SFG response near the achiral chromophore resonances was found to originate from the through-space perturbation of the chiral side chain on the chromophore. Our model successfully explained the observed signal sequence for a series of amino acids and can be generalized to other systems.

We also advanced chirality-sensitive SFG technically. We designed a general inter- 
ference scheme that allows us to measure both the magnitude and the phase of $\chi_{\text {chiral }}^{(2)}$, and for the first time obtain the true chiral SFG spectra that distinguish enantiomers. We also incorporated SFG into a microscopic scheme and showed that SFG microscopy can produce 3D chiral images of sub-micron resolution.

Combined with parity nonconservation, we used SFG to answer the Ozma problems - problems of how chiral information can be transmitted via an achiral channel. It can be seen that as a coherent nonlinear optical process, SFG has a richness in its application that is absent in linear processes, and it provides a new way to measure PNC parameters.

In the second part of the thesis, we describe further developments of sum-frequency vibrational spectroscopy (SFVS) as a surface sensitive technique for achiral systems. We studied the adsorption behavior of leucine molecules at air-water interface. From the polarization-dependent SFVS spectra of the isopropyl headgroup, we deduced how surface density and orientation of adsorbed molecules change with the concentration and the $\mathrm{pH}$ value of the solution. We also found that interfacial water structure is strongly affected by the adsorbed leucine molecules and has a polar orientation reversal when the adsorbed leucine molecules change from anions to cations. We also extended SFVS to C-F normal modes by obtaining the first surface spectrum of C-F modes from shear-aligned poly(tetrafluoroethylene) (PTFE) films. We found that the PTFE polymer chains was aligned along the shearing direction and solved a long-lasting controversy on the symmetry of PTFE normal modes.

In conclusion, our study established SFG as a highly sensitive and powerful tool for chirality detection. We broadened its application in surface science areas of biological 
and industrial interest. 


\section{Bibliography}

[1] Y. R. Shen, The Principles of Nonlinear Optics (John Wiley \& Sons, New York, 1984).

[2] W. Thomson, The Robert Boyle Lecture, Oxford University Junior Scientific Club, May 16, 1893, reprinted in Baltimore Lectures (C. J. Clay \& Sons, London, 1904).

[3] N. Berova, K. Nakanishi, and R. W. Woody, Circular Dichroism: Principles and Applications (Wiley-VCH, Inc., New York, 2000).

[4] D. Craig and T. Thirunamachandran, Molecular Quantum Electrodynamics - An Introduction to Radiation - Molecule Interactions (Dover Publications, Inc., Mineola, New York, 1998).

[5] L. A. Thompson and J. A. Ellman, Chem. Rev. 96, 555 (1996).

[6] D. Figeys and D. Pinto, Anal. Chem. 72, 330A (2000).

[7] B. Alberts, A. Johnson, J. Lewis, M. Raff, K. Roberts, and P. Walter, Molecular Biology of the Cell (Garland Publishing, New York, 2002). 
[8] T. Petralli-Mallow, T. M. Wong, J. D. Byers, H. I. Yee, and J. M. Hicks, J. Phys. Chem. 97, 1383 (1993).

[9] J. D. Byers and J. M. Hicks, Chem. Phys. Lett. 231, 216 (1994).

[10] J. D. Byers, H. I. Yee, and J. M. Hicks, J. Chem. Phys. 101, 6233 (1994).

[11] J. D. Byers, H. I. Yee, T. Petralli-Mallow, and J. M. Hicks, Phys. Rev. B 49, 14643 (1994).

[12] T. P. Petralli-Mallow, A. L. Plant, M. L. Lewis, and J. M. Hicks, Langmuir 16, 5960 $(2000)$.

[13] J. M. Hicks and T. Petralli-Mallow, Appl. Phys. B-Lasers and Optics 68, 589 (1999).

[14] S. Sioncke, T. Verbiest, and A. Persoons, Materials Science \& Engineering R-Reports 42, $115(2003)$.

[15] J. M. Hicks, T. Petrallimallow, and J. D. Byers, Faraday Discussions 341 (1994).

[16] J. A. Giordmaine, Phys. Rev. 138, A1599 (1965).

[17] M. A. Belkin, T. A. Kulakov, K. H. Ernst, L. Yan, and Y. R. Shen, Phys. Rev. Lett. 85, $4474(2000)$.

[18] M. A. Belkin, S. H. Han, X. Wei, and Y. R. Shen, Phys. Rev. Lett. 8711, 113001 (2001).

[19] M. A. Belkin and Y. R. Shen, Phys. Rev. Lett. 91, 213907 (2003). 
[20] N. Ji and Y. R. Shen, J. Am. Chem. Soc. 126, 15008 (2004).

[21] N. Ji and Y. R. Shen, J. Am. Chem. Soc. 127, 12933 (2005).

[22] M. Oh-e, H. Yokoyama, S. Yorozuya, K. Akagi, M. A. Belkin, and Y. R. Shen, Phys. Rev. Lett. 93, 267402 (2004).

[23] S. H. Han, N. Ji, M. A. Belkin, and Y. R. Shen, Phys. Rev. B 66, 165415 (2002).

[24] J. H. Hunt, P. Guyot-Sionnest, and Y. R. Shen, Chem. Phys. Lett. 133, 189 (1987).

[25] P. Guyot-Sionnest, J. H. Hunt, and Y. R. Shen, Phys. Rev. Lett. 59, 1597 (1987).

[26] X. D. Zhu, H. Suhr, and Y. R. Shen, Phys. Rev. B 35, 3047 (1987).

[27] R. W. Boyd, Nonlinear Optics (Academic Press, Boston, 1992).

[28] Y. R. Shen, in Proceedings of the International School of Physics "Enrico Fermi", Course CXX, Frontiers in Laser Spectroscopy, edited by T. W. Hänsch and M. Inguscio (North Holland, Amsterdam, 1994), p. 139.

[29] M. Born and E. Wolf, Principles of Optics (Pergamon Press, New York, 1970).

[30] X. W. Zhuang, P. B. Miranda, D. Kim, and Y. R. Shen, Phys. Rev. B 59, 12632 (1999).

[31] M. A. Belkin, Y. R. Shen, and R. A. Harris, J. Chem. Phys. 120, 10118 (2004).

[32] A. B. Harris, R. D. Kamien, and T. C. Lubensky, Rev. Mod. Phys. 71, 1745 (1999).

[33] M. A. Belkin and Y. R. Shen, Int. Rev. Phys. Chem. 43, 257 (2005). 
[34] M. A. Belkin, Ph.d. thesis, University of California, Berkeley, 2004.

[35] X. Wei, S. C. Hong, A. I. Lvovsky, H. Held, and Y. R. Shen, J. Phys. Chem. B 104, $3349(2000)$.

[36] R. Superfine, J. Y. Huang, and Y. R. Shen, Chem. Phys. Lett. 172, 303 (1990).

[37] D. Kim, Ph.d. thesis, University of California, Berkeley, 1997.

[38] J. B. Biot, Mémoires de l'Académie royale sciences de l'Institut de France 2, 41 (1817).

[39] E. Charney, The Molecular Basis of Optical Activity (John Wiley \& Sons, New York, 1979).

[40] L. D. Barron, Molecular Light Scattering and Optical Activity (Cambridge University Press, Cambridge, UK, 2004).

[41] L. Rosenfeld, Z. Phys. 52, 161 (1928).

[42] M. A. Belkin, Y. R. Shen, and C. Flytzanis, Chem. Phys. Lett. 363, 479 (2002).

[43] E. G. Hohn and O. E. Weigang, J. Chem. Phys. 48, 1127 (1968).

[44] C. J. Cramer and D. G. Truhlar, Chem. Rev. 99, 2161 (1999).

[45] H. J. Maria, D. Larson, McCarvil.Me, and S. P. McGlynn, Acc. Chem. Res. 3, 368 (1970).

[46] C. Fridh, J. Chem. Soc., Faraday Trans. II 74, 190 (1978).

[47] J. G. Kirkwood, J. Chem. Phys. 5, 479 (1937). 
[48] W. Moffitt, R. B. Woodward, A. Moscowitz, W. Klyne, and C. Djerassi, J. Am. Chem. Soc. 83, 4013 (1961).

[49] S. Blanco, A. Lesarri, J. C. Lopez, and J. L. Alonso, J. Am. Chem. Soc. 126, 11675 (2004).

[50] P. Selvarengan and P. Kolandaivel, J. Mol. Struct. Theochem 671, 77 (2004).

[51] E. Tajkhorshid, K. J. Jalkanen, and S. S., J. Phys. Chem. B 102, 5899 (1998).

[52] K. Frimand, H. Bohr, K. J. Jalkanen, and S. Suhai, Chem. Phys. 255, 165 (2000).

[53] A. Csaszar, J. Phys. Chem. 100, 3541 (1996).

[54] B. Lakard, J. Mol. Struct. Theochem 681, 183 (2004).

[55] J. J. P. Stewart, J. Comput. Chem 10, 221 (1989).

[56] J. Fleischhauer, J. Grotzinger, B. Kramer, P. Kruger, A. Wollmer, R. W. Woody, and E. Zobel, Biophys. Chem. 49, 141 (1994).

[57] C. Aleman and J. Puiggali, J. Phys. Chem. B 101, 3441 (1997).

[58] P. Li, Y. X. Bu, and H. Q. Ai, J. Phys. Chem. A 107, 6419 (2003).

[59] HyperChem, HyperChem(TM) professional, Hypercube, Inc., 2003.

[60] R. J. W. Le Fevre, B. J. Orr, and G. L. D. Ritchie, J. Chem. Soc. B - Phys. Org. 273 (1966).

[61] K. G. Denbigh, Trans. Faraday Soc. 36, 936 (1940). 
[62] G. W. Allen, R. S. Armstrong, M. J. Aroney, R. K. Pierens, and A. J. Williams, J. Chem. Soc., Faraday Trans. II 84, 1775 (1988).

[63] A. T. Amos and R. J. Crispin, J. Chem. Phys. 63, 1890 (1975).

[64] P. Bour, J. Kapitan, and V. Baumruk, J. Phys. Chem. A 105, 6362 (2001).

[65] P. A. Snyder, P. M. Vipond, and W. C. Johnson, Biopolymers 12, 975 (1973).

[66] E. U. Condon, W. Altar, and H. Eyring, J. Chem. Phys. 5, 753 (1937).

[67] A. D. Buckingham and P. J. Stiles, Acc. Chem. Res. 7, 258 (1974).

[68] L. I. Katzin and E. Gulyas, J. Am. Chem. Soc. 90, 247 (1968).

[69] L. Fowden, P. M. Scopes, and R. N. Thomas, J. Chem. Soc. C 833 (1971).

[70] H. Nishino, A. Kosaka, G. A. Hembury, K. Matsushima, and Y. Inoue, J. Chem. Soc., Perkin Trans. II 582 (2002).

[71] K. Matsuo, Y. Matsushima, T. Fukuyama, S. Senba, and K. Gekko, Chem. Lett. 826 (2002).

[72] V. Ostroverkhov, G. A. Waychunas, and Y. R. Shen, Phys. Rev. Lett. 94, (2005).

[73] I. Hanazaki and H. Akimoto, J. Am. Chem. Soc. 94, 4102 (1972).

[74] P. Fischer, A. D. Buckingham, K. Beckwitt, D. S. Wiersma, and F. W. Wise, Phys. Rev. Lett. 91, 173901 (2003). 
[75] R. W. Woody, Biochemical Spectroscopy, Vol. 246 of Methods in Enzymology (Academic Press, San Diego, CA, 1995), pp. 34-71.

[76] M. F. Maestre and J. E. Katz, J. Am. Chem. Soc. 21, 1899 (1982).

[77] I. Tinoco, W. Mickols, M. F. Maestre, and C. Bustamante, Ann. Rev. Biophys. BioPhys. Chem. 16, 319 (1987).

[78] K. Claborn, E. Puklin-Faucher, M. Kurimoto, W. Kaminsky, and B. Kahr, J. Am. Chem. Soc. 125, 14825 (2003).

[79] K. Zhang, H. Chang, A. H. Fu, A. P. Alivisatos, and H. Yang, Submitted (2005).

[80] T. Y. F. Tsang, Phys. Rev. A 52, 4116 (1995).

[81] Y. Barad, H. Eisenberg, M. Horowitz, and Y. Silberberg, Appl. Phys. Lett. 70, 922 (1997).

[82] M. Muller, J. Squier, K. R. Wilson, and G. J. Brakenhoff, J. Microscopy - Oxford 191, 266 (1998).

[83] J. F. Ward and G. H. C. New, Phys. Rev. 185, 57 (1969).

[84] T. Verbiest, M. Kauranen, and A. Persoons, J. Chem. Phys. 101, 1745 (1994).

[85] M. Muller, J. Squier, C. A. De Lange, and G. J. Brakenhoff, J. Microscopy - Oxford 197, 150 (2000).

[86] M. Florsheimer, C. Brillert, and H. Fuchs, Langmuir 15, 5437 (1999). 
[87] P. J. Campagnola, M. D. Wei, A. Lewis, and L. M. Loew, Biophys. J. 77, 3341 (1999).

[88] P. J. Campagnola and L. M. Loew, Nature Biotech. 21, 1356 (2003).

[89] M. A. Kriech and J. C. Conboy, J. Am. Chem. Soc. 127, 2834 (2005).

[90] A. Zumbusch, G. R. Holtom, and X. S. Xie, Phys. Rev. Lett. 82, 4142 (1999).

[91] J. X. Cheng, E. Potma, and S. X. Xie, J. Phys. Chem. A 106, 8561 (2002).

[92] M. Gardner, The New Ambidextrous Universe, (W. H. Freeman, New York, 1999).

[93] R. P. Feynman, R. B. Leighton, and M. Sands, The Feynman Lectures on Physics (Addison Wesley Publishing Co., Reading MA, 1965), Vol. 1.

[94] R. A. Harris, in Quantum Dynamics of Molecules, edited by R. G. Woolley (Plenum Publishing Co., New York, 1980).

[95] L. F. Baum, The Wizard of Oz (Reilly \& Lee Co., Chicago, 1956).

[96] A. M. Skelley, J. R. Scherer, A. D. Aubrey, W. H. Grover, R. H. C. Ivester, P. Ehrenfreund, F. J. Grunthaner, J. L. Bada, and R. A. Mathies, PNAS 102, 1041 (2005).

[97] N. Ji, V. Ostroverkhov, M. Belkin, Y.-J. Shiu, and Y. R. Shen, manuscript in preparation .

[98] D. Budker, D. F. Kimball, and D. P. DeMille, Atomic Physics (Oxford University Press, Oxford U.K., 2003). 
[99] J. Crassous, C. Chardonnet, T. Saue, and P. Schwedtfeger, Org. Biomol. Chem. 3, $2218(2005)$.

[100] M. Quack, Angew. Chem. Int. Ed. English, 41, 4618 (2002).

[101] R. A. Harris, Chem. Phys. Lett. 365, 343 (2002).

[102] A. I. MacDermott and R. A. Hegstrom, Chem. Phys. 305, 55 (2004).

[103] J. L. Brash and T. A. Horbett, Proteins at Interfaces (American Chemical Society, Washington, DC, 1987).

[104] A. W. Adamson, Physical Chemistry of Surfaces, 4th ed. ed. (John Wiley \& Sons, New York, 1982).

[105] M. R. Watry and G. L. Richmond, J. Phys. Chem. B 106, 12517 (2002).

[106] C. R. Cantor and P. R. Schimmel, Biophysical Chemistry (W. H. Freeman and Company, San Francisco, 1980).

[107] R. G. Snyder, H. L. Strauss, and C. A. Elliger, J. Phys. Chem. 86, 5145 (1982).

[108] R. A. Macphail, H. L. Strauss, R. G. Snyder, and C. A. Elliger, J. Phys. Chem. 88, 334 (1984).

[109] S. J. Lee, S. W. Han, H. J. Choi, and K. Kim, J. Phys. Chem. B 106, 2892 (2002).

[110] R. A. Macphail, R. G. Snyder, and H. L. Strauss, J. Chem. Phys. 77, 1118 (1982).

[111] B. C. Chow, T. T. Ehler, and T. E. Furtak, Appl. Phys. B 74, 395 (2002). 
[112] M. Himmelhaus, F. Eisert, M. Buck, and M. Grunze, J. Phys. Chem. B 104, 576 (2000).

[113] C. D. Bain, P. B. Davies, T. H. Ong, R. N. Ward, and M. A. Brown, Langmuir 7, $1563(1991)$.

[114] N. Watanabe, H. Yamamoto, A. Wada, K. Domen, C. Hirose, T. Ohtake, and N. Mino, Spectrochimica Acta A 50, 1529 (1994).

[115] K. Wolfrum, H. Graener, and A. Laubereau, Chem. Phys. Lett. 213, 41 (1993).

[116] S. R. Goates, D. A. Schofield, and C. D. Bain, Langmuir 15, 1400 (1999).

[117] G. R. Bell, C. D. Bain, and R. N. Ward, J. Chem. Soc., Faraday Trans. 92, 515 (1996).

[118] C. Hirose, N. Akamatsu, and K. Domen, J. Chem. Phys. 96, 997 (1992).

[119] R. Wüstneck, H. Fiedler, R. Miller, and K. Haage, Langmuir 10, 3966 (1994).

[120] V. B. Fainerman, R. Miller, and H. Mohwald, J. Phys. Chem. B 106, 809 (2002).

[121] L. N. Zhang, C. Zhang, and Y. R. Shen, Macromolecules 36, 3303 (2003).

[122] Q. Du, E. Freysz, and Y. R. Shen, Phys. Rev. Lett. 72, 238 (1994).

[123] H. Suzuki, K. Oiwa, A. Yamada, H. Sakakibara, H. Nakayama, and S. Mashiko, Jpn. J. Appl. Phys. I 34, 3937 (1995).

[124] J. C. Wittmann and P. Smith, Nature 352, 414 (1991). 
[125] P. Damman, M. Dosiere, P. Smith, and J. C. Wittmann, J. Am. Chem. Soc. 117, 1117 (1995).

[126] H. I. Smith, M. W. Geis, C. V. Thompson, and H. A. Atwater, J. Cryst. Growth 63, $527(1983)$

[127] T. Tanaka and M. Ishitobi, J. Phys. Chem. B 106, 564 (2002).

[128] D. Fenwick, K. J. Ihn, F. Motamedi, J. C. Wittmann, and P. Smith, J. Appl. Polym. Sci. 50, 1151 (1993).

[129] Z. Chen, Y. R. Shen, and G. A. Somorjai, Ann. Rev. Phys. Chem. 53, 437 (2002).

[130] P. Dannetun, M. Schott, and M. R. Vilar, Thin Solid Films 286, 321 (1996).

[131] G. Masetti, F. Cabassi, G. Morelli, and G. Zerbi, Macromolecules 6, 700 (1973).

[132] H. Hansma, F. Motamedi, P. Smith, P. Hansma, and J. C. Wittman, Polymer 33, 647 (1992).

[133] D. I. Bower and W. F. Maddams, The Vibrational Spectroscopy of Polymers (Cambridge University Press, New York, 1989).

[134] R. E. Moynihan, J. Am. Chem. Soc. 81, 1045 (1959).

[135] C. J. Peacock, P. J. Hendra, H. A. Willis, and M. E. A. Cudby, J. Chem. Soc. A 2943 (1970).

[136] D. J. Cutler, P. J. Hendra, R. R. Rahalkar, and M. E. A. Cudby, Polymer 22, 726 (1981). 


\title{
Appendix A
}

\section{Sum-frequency generation}

\author{
described by the plane wave
}

\section{approximation and the focused}

\section{Gaussian wave approximation}

As described in Chapter 2, coherent sum-frequency radiation obeys the wave equation

$$
\nabla \times \nabla \times \vec{E}_{s}+\frac{\epsilon}{c^{2}} \frac{\partial^{2} \vec{E}_{s}}{\partial t^{2}}=-\frac{4 \pi}{c^{2}} \frac{\partial^{2} \vec{P}_{s}^{(2)}}{\partial t^{2}}
$$

which can be rewritten as

$$
\nabla\left(\nabla \cdot \vec{E}_{s}\right)-\nabla^{2} \vec{E}_{s}+\frac{\epsilon}{c^{2}} \frac{\partial^{2} \vec{E}_{s}}{\partial t^{2}}=-\frac{4 \pi}{c^{2}} \frac{\partial^{2} \vec{P}_{s}^{(2)}}{\partial t^{2}}
$$


For a source free, isotropic medium,

$$
\nabla \cdot \vec{E}_{s} \propto \nabla \cdot \vec{D}_{s}=0
$$

thus Eq. (A.2) becomes

$$
\nabla^{2} \vec{E}_{s}-\frac{\epsilon}{c^{2}} \frac{\partial^{2} \vec{E}_{s}}{\partial t^{2}}=\frac{4 \pi}{c^{2}} \frac{\partial^{2} \vec{P}_{s}^{(2)}}{\partial t^{2}}
$$

\section{A.1 Collinear geometry}

Let us first focus on the case where the input beams are collinear and propagating along Z laboratory coordinate axis.

\section{A.1.1 Plane wave description}

Considering the input waves as plane waves, we can solve Eq. (A.4) to obtain the sum-frequency signal strength.

For electric field $\vec{E}_{s}(\vec{r}, t)$ and polarization $\vec{P}_{s}^{(2)}(\vec{r}, t)$ of sum-frequency wave, we can write

$$
\begin{aligned}
\vec{E}_{s}(\vec{r}, t) & =\vec{E}_{s}(\mathrm{Z}, t) \\
& =\vec{A}_{s}(\mathrm{Z}) e^{i\left(k_{s} \mathrm{Z}-\omega_{s} t\right)}+c . c . \\
\vec{P}_{s}^{(2)}(\vec{r}, t) & =\vec{P}_{s}^{(2)}(\mathrm{Z}, t) \\
& =\vec{P}_{s}(\mathrm{Z}) e^{i\left[\left(k_{1}+k_{2}\right) \mathrm{Z}-\omega_{s} t\right]}+c . c . .
\end{aligned}
$$

Substituting Eqs. (A.5) and (A.6) into Eq. (A.4), we get

$$
e^{i\left(k_{s} \mathrm{Z}-\omega_{s} t\right)} \frac{\partial^{2} \vec{A}_{s}(\mathrm{Z})}{\partial \mathrm{Z}^{2}}+2 i k_{s} e^{i\left(k_{s} \mathrm{Z}-\omega_{s} t\right)} \frac{\partial \vec{A}_{s}(\mathrm{Z})}{\partial \mathrm{Z}}=-\frac{4 \pi \omega_{s}^{2}}{c^{2}} \vec{P}_{s}(\mathrm{Z}) e^{i\left(k_{1} \mathrm{Z}+k_{2} \mathrm{Z}-\omega_{s} t\right)}
$$


Defining $\Delta k=k_{1}+k_{2}-k_{s}$ as the phase mismatch, Eq. (A.7) becomes

$$
\frac{\partial^{2} \vec{A}_{s}(\mathrm{Z})}{\partial \mathrm{Z}^{2}}+2 i k_{s} \frac{\partial \vec{A}_{s}(\mathrm{Z})}{\partial \mathrm{Z}}=-\frac{4 \pi \omega_{s}^{2}}{c^{2}} \vec{P}_{s}(\mathrm{Z}) e^{i \Delta k \mathrm{Z}}
$$

With the slowly-varying amplitude approximation, in which the transfer of energy among waves is assumed to be only significant when the waves interact over a distance much longer than their wavelength, we have

$$
\left|\frac{\partial^{2} \vec{A}_{s}(\mathrm{Z})}{\partial \mathrm{Z}^{2}}\right| \ll\left|k_{s} \frac{\partial \vec{A}_{s}(\mathrm{Z})}{\partial \mathrm{Z}}\right|
$$

thus obtain

$$
\frac{\partial \vec{A}_{s}(\mathrm{Z})}{\partial \mathrm{Z}}=\frac{2 i \pi \omega_{s}}{c n_{s}} \vec{P}_{s}(\mathrm{Z}) e^{i \Delta k \mathrm{Z}}
$$

For plane incident waves $\omega_{1}$ and $\omega_{2}$, we have

$$
\begin{aligned}
& \vec{E}_{1}(\vec{r}, t)=\vec{A}_{1} e^{i\left(k_{1} \mathrm{Z}-\omega_{1} t\right)}+c . c . \\
& \vec{E}_{2}(\vec{r}, t)=\vec{A}_{2} e^{i\left(k_{2} \mathrm{Z}-\omega_{2} t\right)}+c . c .
\end{aligned}
$$

thus the nonlinear polarization induced by the input waves has

$$
\vec{P}_{s}(Z)=\overleftrightarrow{\chi}^{(2)}: \vec{A}_{1} \vec{A}_{2}
$$

Substituting Eq. (A.11) into Eq. (A.10) and integrating both sides of Eq. (A.10), we get

$$
A_{s}(\mathrm{Z})=\frac{2 i \pi \omega_{s}}{c n_{s}} A_{1} A_{2} \int_{\mathrm{Z}_{0}}^{\mathrm{Z}} \chi^{(2)}(z) e^{i \Delta k z} d z
$$

where $\mathrm{Z}_{0}$ is the starting plane of the nonlinear medium.

If we consider a uniform medium, $\chi^{(2)}(z)$ is a constant and

$$
A_{s}(\mathrm{Z})=\frac{2 i \pi \omega_{s}}{c n_{s}} A_{1} A_{2} \chi^{(2)} \int_{\mathrm{Z}_{0}}^{\mathrm{Z}} e^{i \Delta k z} d z
$$




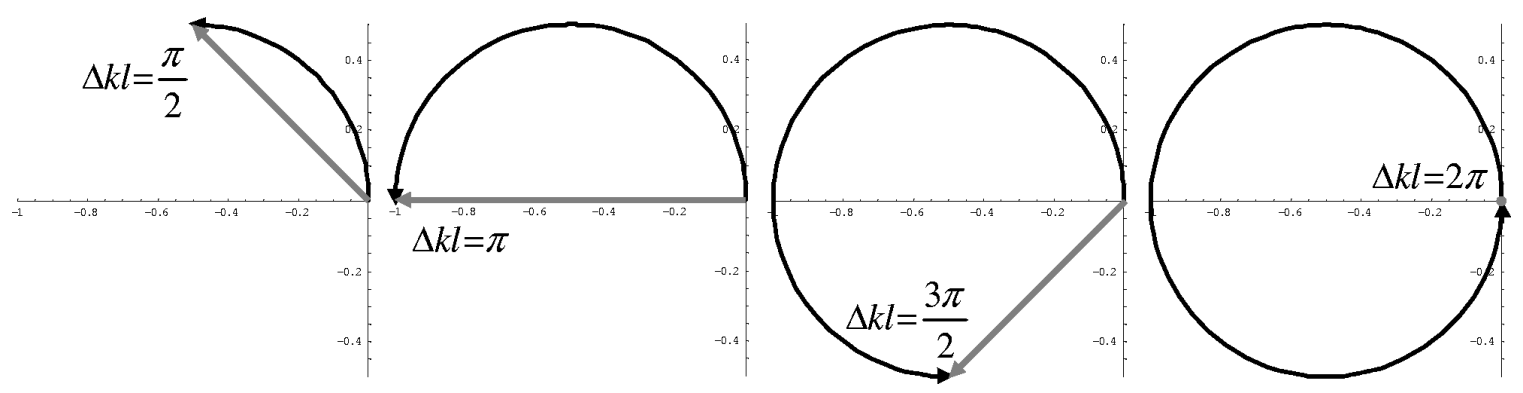

Figure A.1: Curves showing the variation of sum-frequency electric field with interaction length $l$ for planar input waves. From left to right, the interaction length $l$ 's are such that $\Delta k l$ equals to $\pi / 2, \pi, 3 \pi / 2$, and $2 \pi$, respectively. The lengths of the gray arrows reflect the amplitudes of the generated sum-frequency electric field after interaction length $l$.

(i) If the phase-matching is perfect, $\Delta k=0$, then the electric field of sum-frequency output is proportional to the interaction length $l=\mathrm{Z}-\mathrm{Z}_{0}$, and the intensity of sum-frequency output is proportional to $l^{2}$.

(ii) For the case where there is a finite phase mismatch, Eq. (A.13) gives

$$
A_{s}(l)=\frac{2 \pi \omega_{s}}{c n_{s}} A_{1} A_{2} \chi^{(2)}\left(\frac{e^{i \Delta k l}-1}{\Delta k}\right)
$$

from which the concept of coherence length can be derived, as discussed in references [1] and [27]. Here we plot how $A_{s}(l)$ changes with interaction length $l$ on a complex plane. As shown in Fig. A.1, with the increase of $l$, the amplitude of sum-frequency wave first increases, reaches the maximum when $\Delta k l$ equals to $\pi$, then starts to decrease, until totally vanishes when $\Delta k l=2 \pi$. Upon further increase of $l$, sum-frequency wave amplitude retraces the circle in Fig. A.1. 


\section{A.1.2 Focused Gaussian beam description}

In reality, input beams utilized in experiments are never plane waves. They are better described as Gaussian beams. In the following, we present the sum-frequency amplitude solution with focused Gaussian beams propagating along $\mathrm{Z}$ axis as inputs [27].

For simplicity, input Gaussian waves with frequency $\omega_{1}$ and $\omega_{2}$ are assumed to have the same beam waist and divergence with

$$
\begin{aligned}
\vec{E}_{1}(\vec{r}, t) & =\vec{A}_{1}(\vec{r}) e^{i\left(k_{1} \mathrm{Z}-\omega_{1} t\right)}+c . c . \\
& =\vec{A}_{1} \frac{1}{1+i \xi} e^{-\frac{r^{2}}{w_{0}^{2}(1+i \xi)}} e^{i\left(k_{1} \mathrm{Z}-\omega_{1} t\right)}+c . c . \\
\vec{E}_{2}(\vec{r}, t) & =\vec{A}_{2}(\vec{r}) e^{i\left(k_{2} \mathrm{Z}-\omega_{2} t\right)}+c . c . \\
& =\vec{A}_{2} \frac{1}{1+i \xi} e^{-\frac{r^{2}}{w_{0}^{2}(1+i \xi)}} e^{i\left(k_{2} \mathrm{Z}-\omega_{2} t\right)}+c . c .,
\end{aligned}
$$

where $w_{0}$ is the $1 / e$ radius of the field distribution at focal plane $Z=0$, and $\xi=2 Z / b$ is the normalized $\mathrm{Z}$ coordinate in terms of the confocal parameter

$$
b \equiv 2 \pi w_{0}^{2} / \lambda
$$

as schematically shown in Fig. A.2(a).

For sum frequency generated by Gaussian beams, Eq. (A.8) becomes

$$
\frac{\partial^{2} \vec{A}_{s}(\vec{r})}{\partial \mathrm{X}^{2}}+\frac{\partial^{2} \vec{A}_{s}(\vec{r})}{\partial \mathrm{Y}^{2}}+2 i k_{s} \frac{\partial \vec{A}_{s}(\vec{r})}{\partial \mathrm{Z}}=-\frac{4 \pi \omega_{s}^{2}}{c^{2}} \vec{P}_{s}(\vec{r}) e^{i \Delta k \mathrm{Z}}
$$

with the sum-frequency electric field being

$$
\vec{E}_{s}(\vec{r}, t)=\vec{A}_{s}(\vec{r}) e^{i\left(k_{s} \mathrm{Z}-\omega_{s} t\right)}+\text { c.c. }
$$


and the nonlinear polarization as

$$
\vec{P}_{s}^{(2)}(\vec{r}, t)=\vec{P}_{s}(\vec{r}) e^{i\left[\left(k_{1}+k_{2}\right) \mathrm{Z}-\omega_{s} t\right]}+\text { c.c.. }
$$

Substituting

$$
\vec{P}_{s}(\vec{r})=\overleftrightarrow{\chi}^{(2)}: \vec{A}_{1}(\vec{r}) \vec{A}_{2}(\vec{r})
$$

into Eq. (A.17) and adopting the trial solution

$$
A_{s}(\vec{r})=\frac{A_{s}(Z)}{1+i \xi} e^{-\frac{2 r^{2}}{w_{0}^{2}(1+i \xi)}}
$$

one can find $[27]$

$$
A_{s}(\vec{r})=\frac{2 i \pi \omega_{s}}{c n_{s}} A_{1} A_{2} \frac{1}{1+i \xi} e^{-\frac{2 r^{2}}{w_{0}^{2}(1+i \xi)}} \int_{\mathrm{Z}_{0}}^{\mathrm{Z}} \chi^{(2)}(\vec{r}) \frac{e^{i \Delta k z}}{1+2 i z / b} d z
$$

The key difference between the plane wave case (cf. Eq. (A.12)) and Gaussian beam case is the denominator in the integral of Eq. (A.22). The integral can be rewritten as

$$
\int_{\mathrm{Z}_{0}}^{\mathrm{Z}} \frac{\chi^{(2)}(\vec{r})}{\sqrt{1+4 z^{2} / b^{2}}} e^{i \Delta k z} e^{-i \arctan (2 z / b)} d z
$$

We see from the above expression that the contribution from the medium located away from the focal plane decreases in amplitude $\left(\chi^{(2)}(\vec{r}) \rightarrow \frac{\chi^{(2)}(\vec{r})}{\sqrt{1+4 z^{2} / b^{2}}}\right)$, and carries an additional phase $\phi=-\arctan (2 z / b)$.

Similar to the plane wave case, we can plot the change of integral (A.23) with interaction length on a complex plane with the foci of the input waves located at $\mathrm{Z}=0$. For the case of $\Delta k \neq 0$, instead of tracing a circle, (A.23) now traces a spiral, whose shape depends on material susceptibility $\chi^{(2)}(\vec{r})$, focal parameter $b$, and phase mismatch $\Delta k$. 
(a)

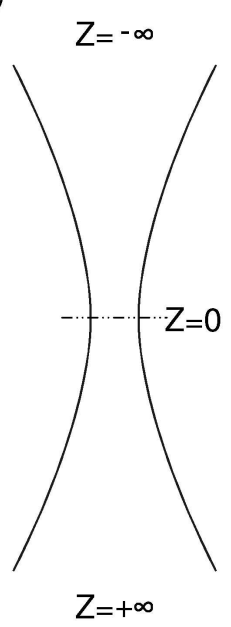

(b)

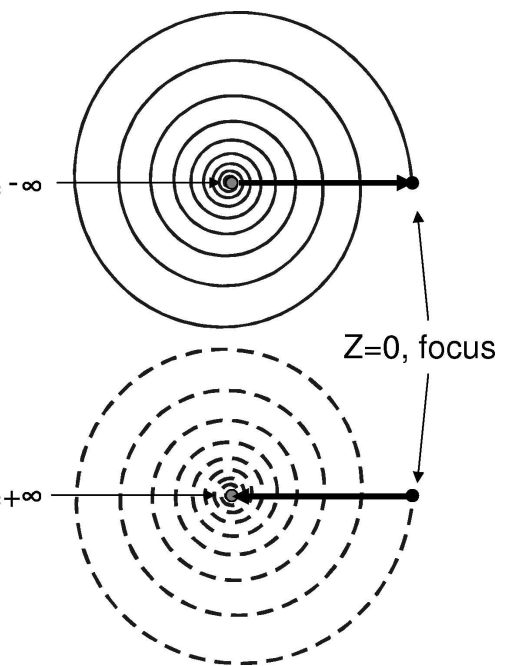

Figure A.2: (a) Incident waves with their focal planes at $\mathrm{Z}=0$. (b) Spirals showing the generation of sum-frequency field in the nonlinear medium from $Z=-\infty$ to $Z=0$ (upper spiral) and $\mathrm{Z}=0$ to $\mathrm{Z}=+\infty$ (lower spiral). The thick black arrows indicate the relative phase and amplitude of the electric fields in two regions.

Here we limit our discussion to a uniform nonlinear material, in which the input beams are moderately focused with a significant phase mismatch so that $b \Delta k \gg 1$ [83].

The contributions to the sum-frequency field from regions on either side of the focus are plotted separately in Fig. A.2(b). The top panel describes SFG from $Z=-\infty$ to $\mathrm{Z}=0$ and the bottom spiral describes the generation from $\mathrm{Z}=0$ to $\mathrm{Z}=+\infty$ with $\pm \infty$ at the centers of the spirals. The arrows overlaying the upper and lower spirals denotes the total fields generated from $-\infty$ to 0 and 0 to $+\infty$, respectively, which have a phase difference of $180^{\circ}$. If the two regions on either side of the focus have same $\chi^{(2)}$ as in Fig. A.2(b), the fields have the same magnitude and the overall generation from $-\infty$ to $+\infty$ is zero. Maximal generation is obtained when the nonlinear medium only occupy the region on one side of 
the focus. Experimentally, this corresponds to make the focal planes of the incident waves coincide with the entrance or exit planes of the nonlinear medium.

\section{A.2 Noncollinear geometry}

In all the experiments described in this thesis, the input beams are not collinear. There are two reasons for such a geometry. Noncollinear geometry produces sum-frequency output that is spatially separated from the incident waves, thus facilitate its detection. Furthermore, to use SFG for chirality detection, one has to use noncollinear geometry in order to probe the chiral components of $\overleftrightarrow{\chi}^{(2)}$

For the noncollinear scheme, the interaction region of two input beams is the volume where the two beams overlap. In principle, one can use mathematical formulism similar to those in the previous section to describe SFG under noncollinear geometry. In the following, however, we present a physical argument that, similar to the collinear Gaussian wave case, to most effectively generate sum-frequency radiation from a bulk medium, the interaction region should be bisected by the surface of the medium.

SFG from a finite interaction region, as schematically shown in Fig. A.3(a), with a dimension $L$ satisfying $\Delta k L \gg 1$ can be approximated to be proportional to an integral of the form

$$
\int_{-\infty}^{Z} \frac{\chi^{(2)}(\vec{r})}{1+f(z)} e^{i \Delta k z} e^{i \phi(z)} d z
$$

where $\mathrm{Z}=0$ is plane B in Fig. A.3(a), which bisects the interaction volume, and $f(z)$ is a real positive function whose value monotonously increases with the increase of $|z|$. Sum- 
(a)

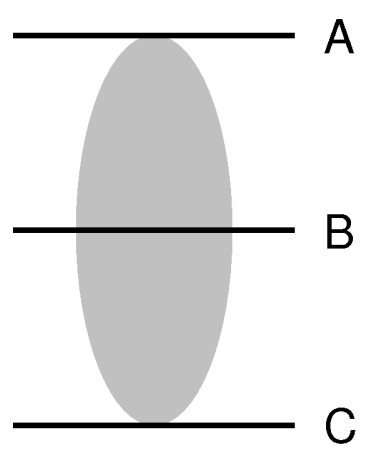

(b)
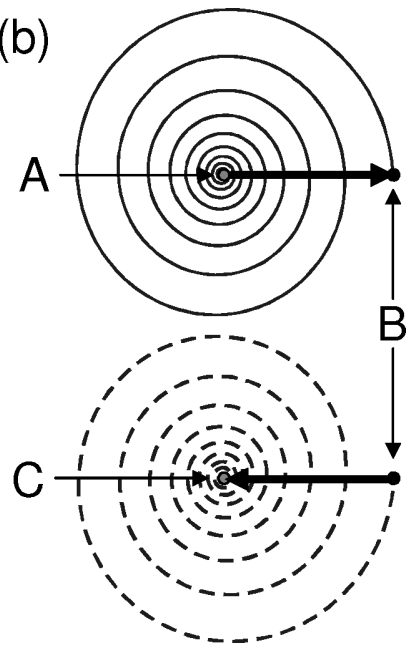

Figure A.3: (a) Interaction volume in nonlinear geometry with three planes A, B, and C defined as its starting, bisecting, and ending planes. (b) Spirals describe the sum-frequency electric fields generated in regions from plane A to plane B (upper spiral) and from plane B to plane C (lower spiral).

frequency field generated in this volume can be represented by spirals similar to those in Fig. A.2. From plane A to plane B, a sum-frequency field is generated, denoted by the black arrow in the upper spiral of Fig. A.3(b). Further interaction in the region from plane B toward plane $\mathrm{C}$ would first decrease the overall sum-frequency field, then ultimately annihilate it. Therefore, experimentally, the maximal sum-frequency signal should be obtained when the two input beams are overlapped at the surface of the bulk medium. This is indeed what we observed. Chiral SFG signal was maximized when the input beams were overlapped at the chiral liquid - cell window interface. 


\section{Appendix B}

\section{Using polarization interference to}

\section{solve the first Ozma problem}

One of the ways to distinguish L and R molecules in SFG is to interfere the achiral response due to electric quadrupole matrix elements, which we call $\chi_{Q}^{(2)}$, with the chiral response $\chi_{\text {chiral }}^{(2)}$. Here, one of the input beams, say $\vec{k}_{1}$, has an electric field $\vec{E}_{1}$ with both $\vec{S}$ and $\vec{P}$ polarization components, which result in chiral and achiral responses simultaneously. Similar to the external electric-field method, for L molecules, sum-frequency intensity is

$$
I_{\mathrm{L}} \propto\left|\vec{S}_{1} \cdot \vec{P}_{2} \times \vec{P}_{3} \chi^{(2)}(\mathrm{L})+\sum_{i, j, k, l}\left(\vec{P}_{i} \cdot \vec{k}_{j}\right)\left(\vec{P}_{k} \cdot \vec{P}_{l}\right) \chi_{Q, i j k l}^{(2)}\right|^{2}
$$

As shown in Fig. 6.2, there are two possible experiment configurations with one of the input beams to the left of the other. With the electric field method, the two different intensities $\left(I_{1}\right.$ and $I_{2}$ in Chapter 6$)$ are used to communicate what we mean by "left" to Wizard of Oz. Similarly, we can use the polarization interferometry method to solve the 

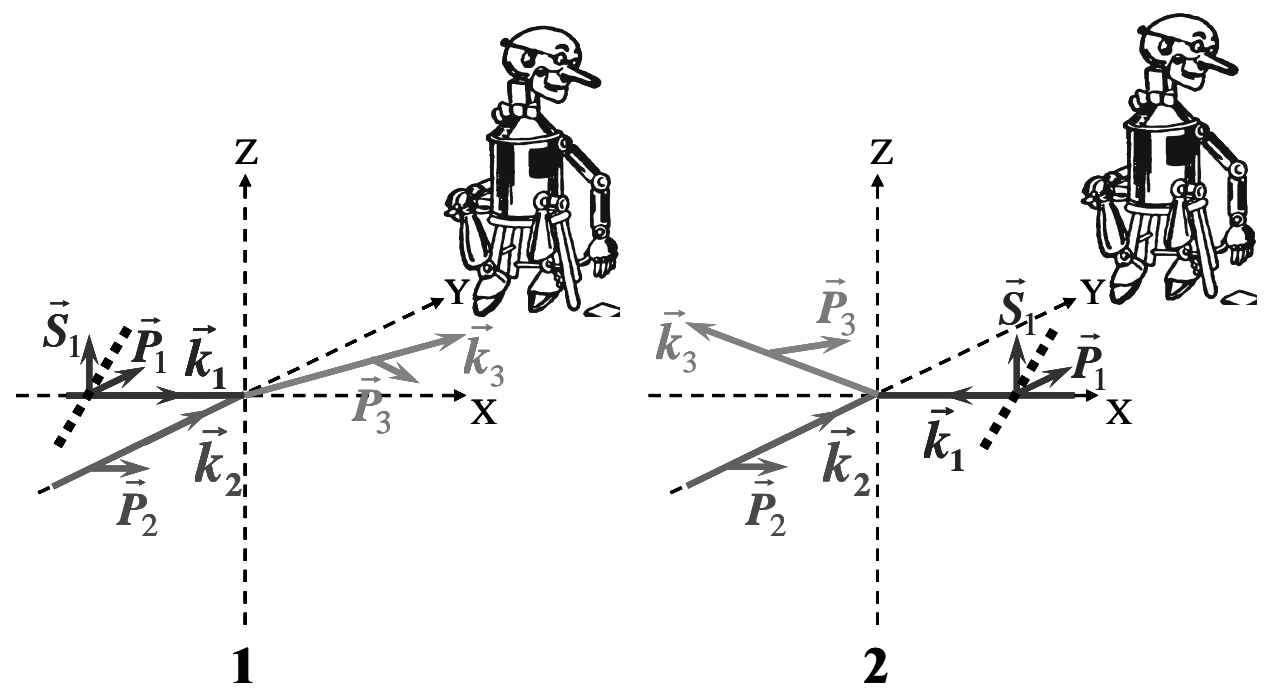

Figure B.1: Two experimental configurations (1 and 2) for wave vectors $\vec{k}_{1}, \vec{k}_{2}$, and $\vec{k}_{3}$, with the latter two waves having $\vec{P}$ polarizations $\left(\vec{P}_{2}\right.$ and $\left.\vec{P}_{3}\right)$. The polarization for $\vec{k}_{1}$ (thick dotted lines) is set to be $45^{\circ}$ away from $\vec{S}_{1}$, with its upper part closer to the tin woodman than its bottom part.

first Ozma problem.

Figure B.1 shows the experimental geometry that can achieve this goal. Here, the tin woodman stands along $\hat{Z}$ direction and perpendicular to the $\hat{X}-\hat{Y}$ plane, which is chosen to be the incidence plane. $\vec{k}_{2}$ is directed along towards the tin woodman. $\vec{k}_{1}$ is set to be perpendicular to $\vec{k}_{2}$, and can be along either $\hat{\mathrm{X}}$ (Configuration 1 ) or $-\hat{\mathrm{X}}$ (Configuration 2). The resulting sum frequency $\vec{k}_{3}=\vec{k}_{1}+\vec{k}_{2}$ is in $\hat{\mathrm{X}}-\hat{\mathrm{Y}}$ plane. The polarization of $\vec{k}_{2}$ and $\vec{k}_{3}$ is set to be $\mathrm{P}$. The polarizer for $\vec{k}_{1}$ is oriented so that the polarization axis has a $45^{\circ}$ angle with $\hat{Z}$, and the upper part of the axis is closer to the tin woodman than the lower part, as shown by the thick dotted lines in Fig. B.1. With angle $\theta$ between $\vec{P}_{2}$ and $\vec{P}_{3}$, 
sum-frequency intensities of configurations 1 and 2 are

$$
\begin{aligned}
& I_{1} \propto\left|\sin \theta \chi^{(2)}(\mathrm{L})+\sum_{i, j, k, l} C_{i j k l} \chi_{Q, i j k l}^{(2)}\right|^{2} \\
& I_{2} \propto\left|-\sin \theta \chi^{(2)}(\mathrm{L})+\sum_{i, j, k, l} C_{i j k l} \chi_{Q, i j k l}^{(2)}\right|^{2}
\end{aligned}
$$

Here $I_{1}$ does not equal to $I_{2}$, and the relationship between them is the same on earth and in Oz. This is true even for general cases where $\vec{k}_{1}$ is not perpendicular to $\vec{k}_{2}$, as long as the polarizer for $\vec{k}_{1}$ is set at the same angle with the upper part closer to the tin woodman in two configurations. As shown in Chapter 6, because these two configurations can be distinguished by their differing sum-frequency intensities, the earth wizard can use this polarization interferometry scheme to tell the Wizard of Oz our definition of "left". 